\title{
THE BOLOCAM GALACTIC PLANE SURVEY. III. CHARACTERIZING PHYSICAL PROPERTIES OF MASSIVE STAR-FORMING REGIONS IN THE GEMINI OB1 MOLECULAR CLOUD
}

\author{
Miranda K. Dunham ${ }^{1}$, Erik Rosolowsky ${ }^{2}$, Neal J. Evans II ${ }^{1}$, Claudia J. Cyganowski ${ }^{3}$, James Aguirre ${ }^{4}$, John Bally ${ }^{5}$, \\ Cara Battersby ${ }^{5}$, Eric Todd Bradley ${ }^{6}$, Darren Dowell ${ }^{7}$, Meredith Drosback ${ }^{8}$, Adam Ginsburg ${ }^{5}$, Jason GlenN $^{5}$, \\ Paul Harvey $^{1,5}$, Manuel Merello ${ }^{1}$, Wayne Schlingman ${ }^{9}$, Yancy L. Shirley ${ }^{9}$, Guy S. Stringfellow ${ }^{5}$, \\ JoSh WALAWENDER ${ }^{10}$, AND JONATHAN P. Williams ${ }^{11}$ \\ ${ }^{1}$ Department of Astronomy, The University of Texas at Austin, 1 University Station C1400, Austin, TX 78712-0259, USA; nordhaus@astro.as.utexas.edu \\ ${ }^{2}$ University of British Columbia, Okanagan, 3333 University Way, Kelowna BC V1V 1V7, Canada \\ ${ }^{3}$ Department of Astronomy, University of Wisconsin, Madison, WI 53706, USA \\ ${ }^{4}$ Department of Physics and Astronomy, University of Pennsylvania, Philadelphia, PA 19104, USA \\ ${ }^{5}$ CASA, University of Colorado, 389-UCB, Boulder, CO 80309, USA \\ ${ }^{6}$ Department of Physics, University of Central Florida, 4000 Central Florida Boulevard, Orlando, FL 32816-2385, USA \\ 7 Jet Propulsion Laboratory, California Institute of Technology, 4800 Oak Grove Drive, Pasadena, CA 91104, USA \\ ${ }^{8}$ Department of Astronomy, University of Virginia, P.O. Box 400325, Charlottesville, VA 22904, USA \\ ${ }^{9}$ Steward Observatory, University of Arizona, 933 North Cherry Avenue, Tucson, AZ 85721, USA \\ ${ }^{10}$ Institute for Astronomy, University of Hawaii, 640 N. Aohoku Place, Hilo, HI 96720, USA \\ ${ }^{11}$ Institute for Astronomy, University of Hawaii, 2680 Woodlawn Drive, Honolulu, HI 96822, USA \\ Received 2009 August 13; accepted 2010 May 13; published 2010 June 23
}

\begin{abstract}
We present the $1.1 \mathrm{~mm}$ Bolocam Galactic Plane Survey (BGPS) observations of the Gemini OB1 molecular cloud complex, and targeted $\mathrm{NH}_{3}$ observations of the BGPS sources. When paired with molecular spectroscopy of a dense gas tracer, millimeter observations yield physical properties such as masses, radii, mean densities, kinetic temperatures, and line widths. We detect 34 distinct BGPS sources above $5 \sigma=0.37 \mathrm{Jy}^{\text {beam }}{ }^{-1}$ with corresponding $5 \sigma$ detections in the $\mathrm{NH}_{3}(1,1)$ transition. Eight of the objects show water maser emission $(20 \%)$. We find a mean millimeter source FWHM of $1.12 \mathrm{pc}$ and a mean gas kinetic temperature of $20 \mathrm{~K}$ for the sample of 34 BGPS sources with detections in the $\mathrm{NH}_{3}(1,1)$ line. The observed $\mathrm{NH}_{3}$ line widths are dominated by non-thermal motions, typically found to be a few times the thermal sound speed expected for the derived kinetic temperature. We calculate the mass for each source from the millimeter flux assuming the sources are isothermal and find a mean isothermal mass within a $120^{\prime \prime}$ aperture of $230 \pm 180 M_{\odot}$. We find a total mass of $8400 M_{\odot}$ for all BGPS sources in the Gemini OB1 molecular cloud, representing $6.5 \%$ of the cloud mass. By comparing the millimeter isothermal mass to the virial mass calculated from the $\mathrm{NH}_{3}$ line widths within a radius equal to the millimeter source size, we find a mean virial parameter $\left(M_{\mathrm{vir}} / M_{\mathrm{iso}}\right)$ of $1.0 \pm 0.9$ for the sample. We find mean values for the distributions of column densities of $1.0 \times 10^{22} \mathrm{~cm}^{-2}$ for $\mathrm{H}_{2}$, and $3.0 \times 10^{14} \mathrm{~cm}^{-2}$ for $\mathrm{NH}_{3}$, giving a mean $\mathrm{NH}_{3}$ abundance of $3.0 \times 10^{-8}$ relative to $\mathrm{H}_{2}$. We find volume-averaged densities on the order of $10^{3}-10^{4} \mathrm{~cm}^{-3}$. The sizes and densities suggest that in the Gem OB1 region the BGPS is detecting the clumps from which stellar clusters form, rather than smaller, higher density cores where single stars or small multiple systems form.
\end{abstract}

Key words: dust, extinction - ISM: clouds - ISM: individual objects (Gem OB1) - radio lines: ISM - stars: formation

Online-only material: color figure set

\section{INTRODUCTION}

The availability of millimeter bolometer arrays has made possible large-scale, blind surveys of the cold dust most closely associated with star formation (e.g., Enoch et al. 2007; Motte et al. 2007; Aguirre et al. 2010; Schuller et al. 2009). Such blind millimeter continuum surveys can identify the clumps where massive stars and clusters are born without the requirement of a signpost of massive star formation, such as maser emission (e.g., Palla et al. 1991; Plume et al. 1992, 1997; Beuther et al. 2002; Mueller et al. 2002; Shirley et al. 2003), radio continuum emission (e.g., Ramesh \& Sridharan 1997; Sridharan et al. 2002), infrared sources (e.g., Molinari et al. 2000; Kumar et al. 2006; Kumar \& Grave 2007; Robitaille et al. 2008), or infrared colors (Wood \& Churchwell 1989). Infrared dark clouds (IRDCs; regions of high density seen in absorption against the diffuse mid-IR background, which are thought to represent the earliest stages of massive star formation) provide another way of finding regions of massive star formation (Rathborne et al. 2006;
Jackson et al. 2008), but only if they lie in front of extended midIR emission. However, IRDCs are seen in emission at millimeter wavelengths, allowing the Bolocam Galactic Plane Survey (BGPS) to detect the earliest stages of massive star formation at larger distances where a significant mid-IR background is absent or too much foreground mid-IR emission is present.

Galaxy-wide millimeter continuum surveys, in conjunction with distance information, will allow us to determine the physical properties of a vast number of sources and begin answering some important questions. What is the impact of environment on star formation? How do the physical properties of clumps, such as size, mass, and mean density vary as a function of Galactocentric radius and proximity to a spiral arm? Answering these questions will provide a greater understanding of star formation in our own Galaxy. Also, by characterizing the distribution of dense gas clumps in our own galaxy, we can better understand the aggregate emission of clumps in distant galaxies where the dense gas structures cannot be resolved. 
In this paper, we refer to clumps as distinct bound regions within molecular clouds that will form entire stellar clusters, and cores as the regions within clumps that will form a single star or small multiple system (e.g., Williams et al. 2000). Clumps can be characterized with masses ranging from 50 to $500 M_{\odot}$, sizes ranging from 0.3 to $3 \mathrm{pc}$, mean densities ranging from $10^{3}$ to $10^{4} \mathrm{~cm}^{-3}$, and gas temperatures ranging from 10 to $20 \mathrm{~K}$, while starless cores can be characterized by masses from 0.5 to $5 M_{\odot}$, sizes of $0.03-0.2 \mathrm{pc}$, mean densities of $10^{4}-10^{5} \mathrm{~cm}^{-3}$, and gas temperatures between 8 and $12 \mathrm{~K}$ (Bergin \& Tafalla 2007).

The BGPS is one of the first millimeter surveys of the northern Galactic plane (Aguirre et al. 2010), and has cataloged 8358 sources (Rosolowsky et al. 2010). The BGPS consists of two parts: a blind survey of the inner Galaxy and a targeted survey of well-known star-forming regions in the outer Galaxy. The inner Galaxy survey coverage is continuous over $-10^{\circ}<l<90.5$, and the outer Galaxy coverage includes IC 1396, the W3/4/ 5 region, $4 \mathrm{deg}^{2}$ toward the Perseus Arm (near NGC 7538), and the Gemini OB1 molecular cloud. With a large range in Galactic longitude, the BGPS provides the opportunity to study star formation in drastically different environments.

Millimeter continuum emission detected in the BGPS can arise from all objects along a line of sight, from a few hundred pc to the other side of the Galaxy; distances to BGPS sources are not known a priori. With recent improvements to kinematic models of the Galaxy (e.g., Pohl et al. 2008) and very long baseline interferometry (VLBI) parallax distances (Reid et al. 2009), radial velocities can yield kinematic distances. Molecular spectral lines also provide information regarding the kinematics within each source. Ammonia $\left(\mathrm{NH}_{3}\right)$, in particular, is an excellent tracer of dense gas which also provides information regarding the line of sight velocity dispersion, kinetic gas temperature, column density, and mass (e.g., Ho \& Townes 1983; Zinchenko et al. 1997; Swift et al. 2005; Rosolowsky et al. 2008; Foster et al. 2009). Pairing the $1.1 \mathrm{~mm}$ continuum data with $\mathrm{NH}_{3}$ observations allows complete characterization of both the gas and dust properties (e.g., Rosolowsky et al. 2008; Foster et al. 2009).

In this paper, we present the results of an $\mathrm{NH}_{3}$ survey of the BGPS sources in the Gemini OB1 molecular cloud. In Section 2, we provide an overview of the structure and previous observations of the Gemini OB1 molecular cloud. We briefly describe the BGPS and $\mathrm{NH}_{3}$ surveys and data reduction in Section 3, and present the parameter estimation methods in Section 4. In Section 5, we discuss the basic results of the BGPS and $\mathrm{NH}_{3}$ in the Gemini OB1 molecular cloud, and in Section 6 we discuss the derived physical properties in detail. We compare our results to previous studies in Section 7.1, and explore the effects of distance on the derived properties in Section 7.2. Finally, we present a summary in Section 8. In future work, we will present Green Bank Telescope (GBT)+BGPS observations of sources at low Galactic longitudes.

Studying the Gem OB1 region is particularly useful because the BGPS sources in this region are located in the same molecular complex and thus have similar distances (Carpenter et al. 1995a, 1995b). We are able to investigate the physical properties of this subset of BGPS sources without the ambiguity introduced by a large range of distances seen for the sample as a whole. This paper will serve as a Galactic anti-center comparison for a study of $\mathrm{NH}_{3}$ observations of BGPS sources at a range of Galactic longitudes in the inner Galaxy (M. K. Dunham et al. 2010, in preparation).

\section{GEMINI OB1 MOLECULAR CLOUD}

Gemini OB1 is an OB association centered near $(l, b)=$ $\left(189^{\circ}, 1^{\circ}\right)$, which was first identified by Morgan et al. (1953). It became known as Gem OB1 when Humphreys (1978) compiled a list of the brightest OB stars in the Milky Way, cataloging $20 \mathrm{OB}$ stars as belonging to the association. Many groups have studied the association and found distances ranging from $1.4 \mathrm{kpc}$ to $1.9 \mathrm{kpc}$ (Crawford et al. 1955; Hardie et al. 1960; Georgelin et al. 1973; Humphreys 1978). There is also a significant spread in age amongst the association members, with ages ranging from 2 Myr (Grasdalen \& Carrasco 1975) to $10 \mathrm{Myr}$ (Barbaro et al. 1969).

The Gemini OB1 molecular cloud was first identified as an enhancement of emission in the Columbia ${ }^{12} \mathrm{CO}$ survey of the Galactic plane (Dame et al. 1987), and spans a $6^{\circ} \times 6^{\circ}$ area on the sky (Huang \& Thaddeus 1986). There are many star-forming regions in the Gem OB 1 molecular cloud, including well-known H II regions S247, S252, the group S254, S255, S256, S257, and S258 (hereafter referred to as S254-258), S259, S261 (Sharpless 1959), and BFS 52 as well as a supernova remnant, IC 443. Reid et al. (2009) used the VLBA to measure the parallax of methanol masers in S252 and found a distance of $2.10 \pm 0.026 \mathrm{kpc}$, which we adopt in this study.

Studies of ${ }^{12} \mathrm{CO}$ and ${ }^{13} \mathrm{CO}$ in the Gem OB1 molecular cloud show abundant arc and ring-like filamentary structure (Carpenter et al. 1995a; Kömpe et al. 1989). Carpenter et al. (1995a) mapped $32 \mathrm{deg}^{2}$ of the Gem OB1 molecular cloud in ${ }^{12} \mathrm{CO}(J=1-0)$ and ${ }^{13} \mathrm{CO}(J=1-0)$ with $50^{\prime \prime}$ sampling and $45^{\prime \prime}$ and $50^{\prime \prime}$ resolution, respectively. They suggest that the filamentary structure is comprised of molecular material which has been swept-up by the expanding $\mathrm{H}$ II regions and wind blown bubbles. They conclude that the high column density regions where star formation is currently occurring were formed through the interaction of newly formed stars and the surrounding molecular material. Carpenter et al. (1995a) split the cloud into seven separate regions and find that three regions (S247, S252, and S254-258) contain active star formation based on higher $\mathrm{H}_{2}$ column densities $\left(>10^{22} \mathrm{~cm}^{-2}\right)$ and warmer kinetic temperatures (10-30 K).

Carpenter et al. (1995b) identified 11 clumps within the ${ }^{12} \mathrm{CO}$ filaments using $\operatorname{CS}(J=2-1)$ observations (55" resolution), all with masses greater than $100 M_{\odot}$. They found that at least eight of the 11 clumps have associated IRAS point sources. Carpenter et al. (1995b) also conducted a near-infrared imaging survey of a $30^{\prime} \times 45^{\prime}$ area surrounding S247, including three of the CS clumps. They found that each of the three clumps contains a cluster of stars in the near-infrared images.

Chavarría et al. (2008) identified 510 young stellar objects (YSOs) with near- or mid-IR excess in the S254-258 complex using a combination of Spitzer IRAC and near-infrared data. They classify 87 Class I sources, and 165 Class II sources, and find that $80 \%$ of the YSOs are in clusters surrounded by a more evenly distributed population.

Figure 1 shows the extinction maps of Dobashi et al. (2005) for the Gemini OB1 molecular cloud. OB association members are shown as stars (Humphreys 1978), H II regions (Sharpless $1959)$ and IC 443 are shown as circles with sizes corresponding to the source size. The extent of the ${ }^{12} \mathrm{CO}$ and ${ }^{13} \mathrm{CO}$ maps of Carpenter et al. (1995a) is marked by the dashed line, while the solid line shows the extent of the BGPS map presented in this paper. 


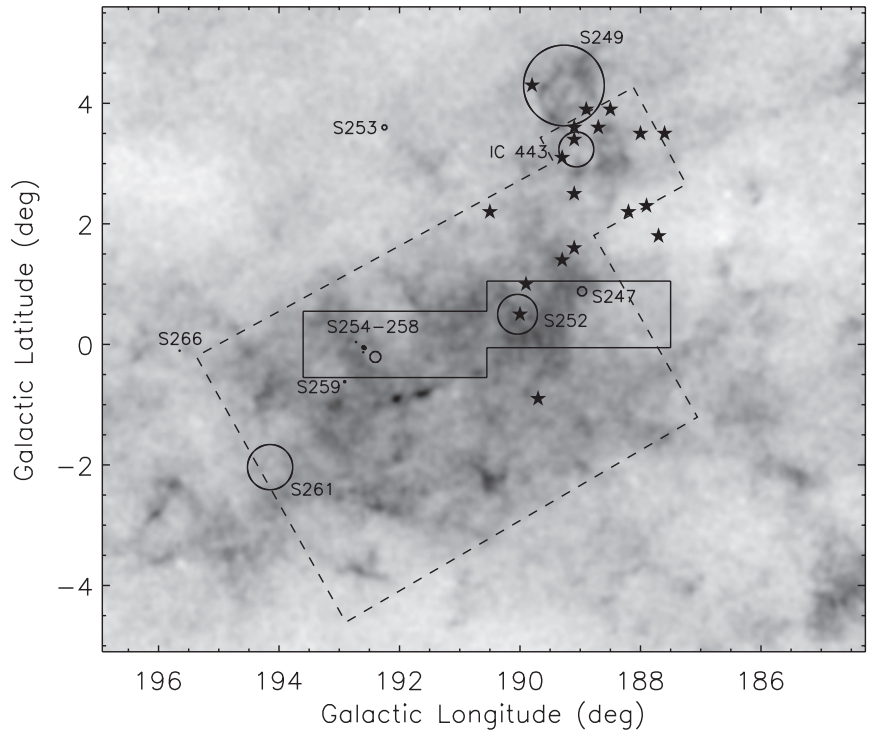

Figure 1. Gemini OB1 molecular cloud. Extinction maps of Dobashi et al (2005) are shown in inverted gray scale ranging from -0.5 to $4.0 \mathrm{mag}$. The $\mathrm{OB}$ association members (Humphreys 1978) are shown as stars. The H II regions (Sharpless 1959) and IC 443 are shown as circles sized to match the extent of the region. The dashed line marks the area mapped in ${ }^{12} \mathrm{CO}$ and ${ }^{13} \mathrm{CO}$ by Carpenter et al.(1995a), and the solid line marks the extent of the $1.1 \mathrm{~mm}$ BGPS maps.

\section{SURVEYS}

\subsection{Bolocam 1.1 mm Galactic Plane Survey}

The BGPS ${ }^{12}$ has surveyed approximately $170 \mathrm{deg}^{2}$ of the Galactic plane using the Bolocam instrument on the Caltech Submillimeter Observatory ${ }^{13}$ (CSO) on Mauna Kea. Aguirre et al. (2010) present specific details regarding the BGPS methods and Rosolowsky et al. (2010) detail the BGPS catalog. The images and catalog have been made public and are hosted by the Infrared Processing and Analysis Center (IPAC) via the NASA/IPAC Infrared Science Archive ${ }^{14}$.

\subsubsection{Observations}

The Gem OB1 region was observed in two $3^{\circ} \times 1^{\circ}$ groups of observations, centered at $(l, b)=\left(189^{\circ}, 0.5\right)$ and $\left(192^{\circ}, 0^{\circ}\right)$, with a shallow observation centered between the two main groups to aid in mosaicing the images (see Figure 2). The centers were selected to place the most active star-forming regions (S247, S252, and S254-258) within our mapped region. Observations were carried out at the CSO as part of the BGPS between 2007 September 9 and 13. The survey used the Bolocam

\footnotetext{
12 See http://milkyway.colorado.edu/bgps/.

13 The Caltech Submillimeter Observatory is supported by the NSF.

14 See http://irsa.ipac.caltech.edu/data/BOLOCAM_GPS/.
}

instrument, ${ }^{15}$ which has a hexagonal array of 144 bolometers. The Bolocam filter is centered at $268 \mathrm{GHz}$ with a bandwidth of $45 \mathrm{GHz}$, and was designed to exclude the ${ }^{12} \mathrm{CO}(2 \rightarrow 1)$ emission line. Each bolometer beam is well approximated by a Gaussian with an FWHM of 31", and the bolometer separation results in an instantaneous field of view of 7'.5. For the BGPS, the effective beam size after processing many scans is $33^{\prime \prime}$ for the current release (Aguirre et al. 2010). For more instrument specific details, see Glenn et al. (2003) and Haig et al. (2004). Observations were obtained using the raster scan mode, with alternating scans directed along $l$ and $b$. The scan speed was $120^{\prime \prime} \mathrm{s}^{-1}$, and chopping was not utilized in these observations, thereby retaining some sensitivity to large-scale structure up to the angular size of the array (see Aguirre et al. 2010 for more detailed analysis).

\subsubsection{Reduction}

At millimeter wavelengths, the detected signal is dominated by the atmosphere, making the sky cleaning algorithm extremely important. Bolocam's individual bolometer beams overlap substantially in the lower atmosphere such that they probe similar columns of atmosphere, facilitating sky subtraction. The iterative mapping method utilizes principal component analysis (PCA) and considers all observations for a given field simultaneously. In our iterative mapping method, the temporally correlated signal is first subtracted from the time streams. This subtraction removes both the atmosphere and all astrophysical structure larger than the array field of view. The remaining time stream, which in principle should contain only astrophysical signal, is then mapped with $7^{\prime \prime} .2 \times 7^{\prime \prime} .2$ pixels, deconvolved from the beam, and returned to a time stream. The time stream containing only astrophysical signal is subtracted from the original to produce a third time stream (summing the subtracted astrophysical signals to make a source map) that represents an estimate of the noise. The above procedure is then repeated for 50 iterations on the deconvolved astrophysical signal time stream to produce the final map. This procedure can be fine-tuned to balance the retention of large-scale structure against production of artifacts (such as negative bowls) around bright sources. The iterative mapping method resulted in a $1 \sigma \mathrm{rms}$ noise of $0.070 \mathrm{Jy} \mathrm{beam}^{-1}$ in the Gemini OB1 image. For more specific information on the BGPS reduction, see Aguirre et al. (2010).

\subsubsection{Images}

The iteratively mapped BGPS image is shown in Figure 2. The millimeter sources in our maps are highly clustered and are found in three groups, while the remainder of the image is strikingly devoid of millimeter emission. This clustering is

${ }^{15}$ See http://www.cso.caltech.edu/bolocam/.

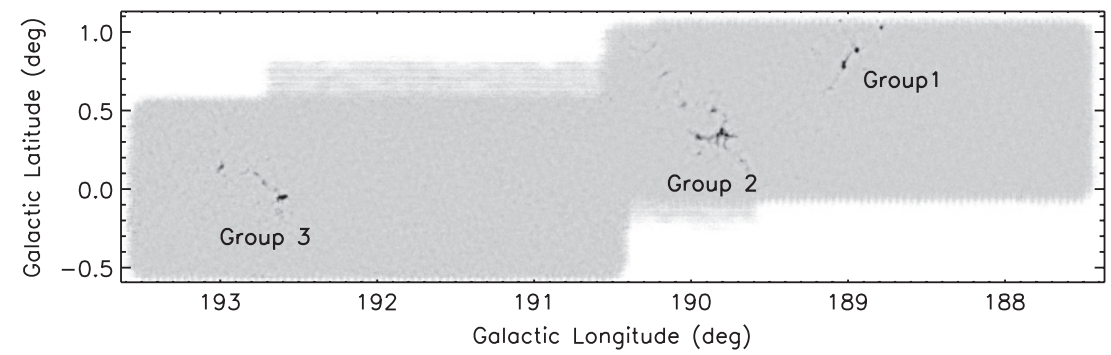

Figure 2. BGPS $1.1 \mathrm{~mm}$ continuum image of the Gemini OB 1 molecular cloud shown in an inverted, linear gray scale ranging from -0.2 to $0.75 \mathrm{Jy}^{\mathrm{b}}$ beam ${ }^{-1}$. Groups 1-3 are shown in more detail in following figures. 


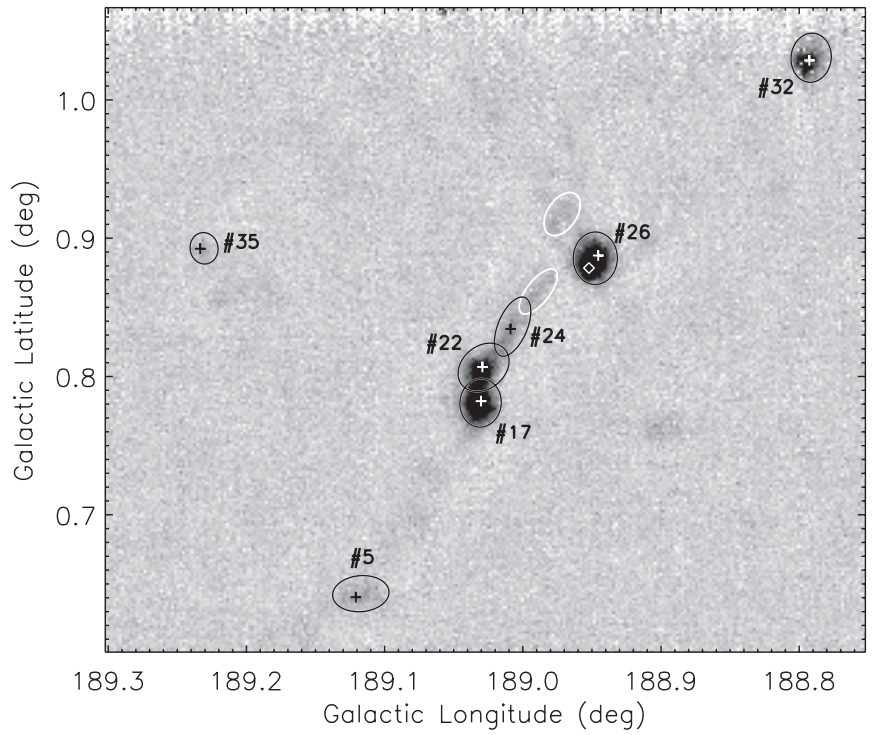

Figure 3. BGPS image of the region marked group 1 in Figure 2 in inverted gray scale ranging from -0.2 to $0.75 \mathrm{Jy} \mathrm{beam}^{-1}$. The BGPS sources with $\mathrm{NH}_{3}$ spectra are shown as black ellipses, while BGPS sources without $\mathrm{NH}_{3}$ spectra are shown as white ellipses. The $\mathrm{NH}_{3}$ pointings are shown as crosses and diamonds. Crosses denote pointings closest to the peak of the millimeter emission, while diamonds denote additional $\mathrm{NH}_{3}$ pointings within each BGPS source.

in contrast to the inner Galaxy regions also included in the BGPS, where a similarly sized image would contain hundreds of sources (see Aguirre et al. 2010; Bally et al. 2010). The three groups correspond to S247 (referred to as group 1; Figure 3), S252 (referred to as group 2; Figure 4), and S254-258 (referred to as group 3; Figure 5).

\section{2. $\mathrm{NH}_{3}$ Survey of BGPS Sources}

The $\mathrm{NH}_{3}$ pointings are displayed in Figures $3-5$ as crosses, diamonds, and asterisks. Since the $\mathrm{NH}_{3}$ pointings were chosen prior to completion of the BGPS catalog, they do not necessarily correspond to the peak positions of the millimeter emission determined by the source extraction algorithm (see Section 4.1). Crosses mark the positions closest to the peak of the millimeter emission, diamonds mark extra $\mathrm{NH}_{3}$ pointings within each source, and the asterisks mark the positions of $\mathrm{NH}_{3}$ pointings that correspond to a millimeter source but do not have a detection in any of the $\mathrm{NH}_{3}$ transitions.

\subsubsection{Observations}

We observed $56 \mathrm{NH}_{3}$ pointings in the Gem OB1 region using the Robert F. Byrd GBT ${ }^{16}$ on 2008 February 14 and 15 in two separate blocks totaling $3.6 \mathrm{hr}$. The $23 \mathrm{GHz}$ zenith opacity for the February 14 observations was $\tau_{23} \sim 0.04$ and for February 15, $\tau_{23} \sim 0.11$. The observations were broken into two minute integrations of individual targets. We used Feed 1 of the high-frequency $K$-band receiver on the GBT as the front end for the observations. The GBT spectrometer served as the back end, which we configured to observe both polarizations of the feed with $50 \mathrm{MHz}$ bandwidth for four separate intermediate frequencies (IFs). The total bandwidth was sampled in 8192 channels for a $6.1 \mathrm{kHz}$ $\left(\sim 0.08 \mathrm{~km} \mathrm{~s}^{-1}\right)$ channel width. We used two different sets of

\footnotetext{
16 The GBT is operated by the National Radio Astronomy Observatory, which is a facility of the National Science Foundation, operated under cooperative agreement by Associated Universities, Inc.
}

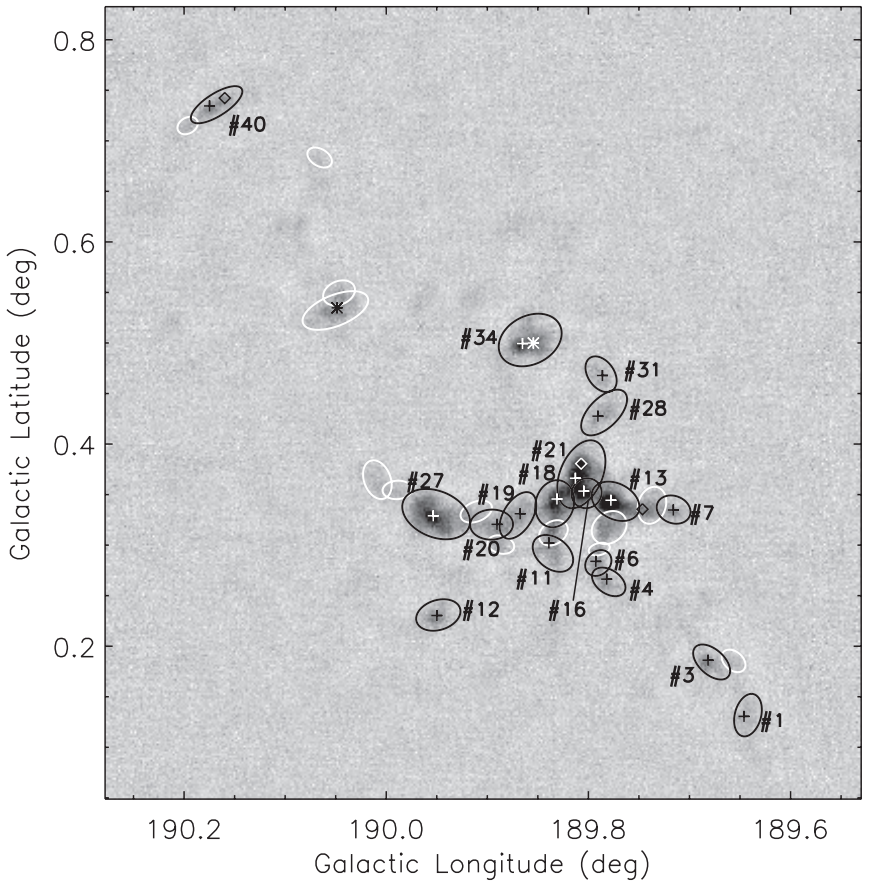

Figure 4. BGPS image of the region marked group 2 in Figure 2 in inverted gray scale ranging from -0.2 to $0.75 \mathrm{Jy} \mathrm{beam}^{-1}$. The BGPS sources with $\mathrm{NH}_{3}$ spectra are shown as black ellipses, while BGPS sources without $\mathrm{NH}_{3}$ spectra are shown as white ellipses. The $\mathrm{NH}_{3}$ pointings are shown as crosses, diamonds, and asterisks. Crosses denote pointings closest to the peak of the millimeter emission, diamonds denote additional $\mathrm{NH}_{3}$ pointings within each BGPS source, and asterisks denote non-detections. Source ellipses can overlap since these are the moment approximations to source sizes rather than the boundaries established by the extraction algorithm.

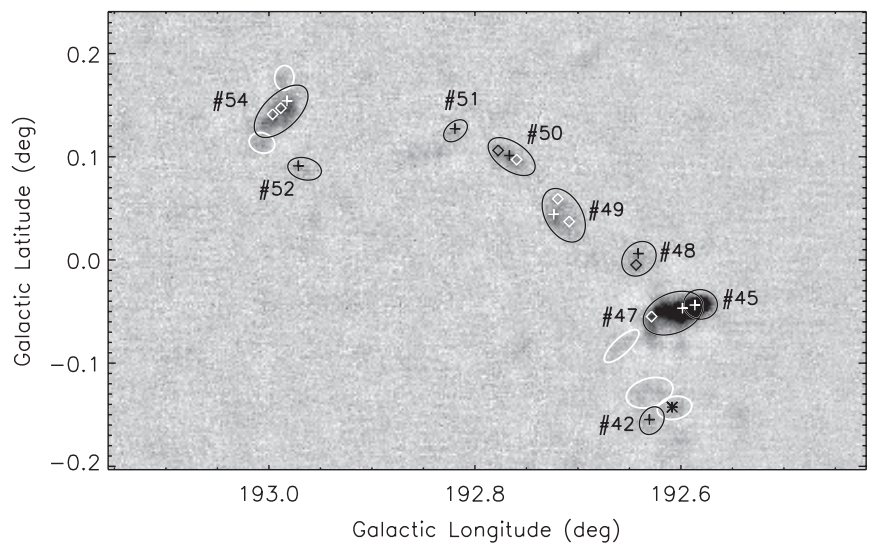

Figure 5. BGPS image of the region marked group 3 in Figure 2 in inverted gray scale ranging from -0.2 to $0.75 \mathrm{Jy}$ beam $^{-1}$. The BGPS sources with $\mathrm{NH}_{3}$ spectra are shown as black ellipses, while BGPS sources without $\mathrm{NH}_{3}$ spectra are shown as white ellipses. The $\mathrm{NH}_{3}$ pointings are shown as crosses, diamonds, and asterisks. Crosses denote pointings closest to the peak of the millimeter emission, diamonds denote additional $\mathrm{NH}_{3}$ pointings within each BGPS source, and asterisks denote non-detections. Source ellipses can overlap since these are the moment approximations to source sizes rather than the boundaries established by the extraction algorithm.

IFs. All scans contained the $\mathrm{NH}_{3}(1,1)(23.6944955(1) \mathrm{GHz})$ and $\mathrm{NH}_{3}(2,2)(23.7226333(1) \mathrm{GHz})$ lines. Observations on February 14 (10 pointings) also included the $\mathrm{C}_{2} \mathrm{~S}\left(N_{J}=2_{1} \rightarrow 1_{0}\right.$, 22.344033(1) $\mathrm{GHz}$, Yamamoto et al. 1990) and $\mathrm{NH}_{3}(4,4)$ (24.1394169(1) GHz) lines. All $\mathrm{NH}_{3}$ frequencies were taken from Lovas \& Dragoset (2003). There were no significant detections of the $\mathrm{C}_{2} \mathrm{~S}$ line and only one marginal detection of the $\mathrm{NH}_{3}(4,4)$ line, so the spectrometer configuration was changed 
Table 1

Mean rms for Spectral Line Observations

\begin{tabular}{lcc}
\hline \hline \multicolumn{1}{c}{ Line } & Mean rms $(\mathrm{mK})$ & St. Dev. rms $(\mathrm{mK})$ \\
\hline $\mathrm{NH}_{3}(1,1)$ & 82 & 21 \\
$\mathrm{NH}_{3}(2,2)$ & 81 & 21 \\
$\mathrm{NH}_{3}(3,3)$ & 97 & 11 \\
$\mathrm{NH}_{3}(4,4)$ & 52 & 1 \\
$\mathrm{H}_{2} \mathrm{O}$ & 172 & 26 \\
$\mathrm{C}_{2} \mathrm{~S}$ & 67 & 4 \\
\hline
\end{tabular}

for the following days' observations. The observations on February 15 included the $\mathrm{H}_{2} \mathrm{O}(22.344033 \mathrm{GHz})$, and $\mathrm{NH}_{3}(3,3)$ $(23.8701279(5) \mathrm{GHz})$ transitions in the remaining two IFs. Ten pointings were observed on 2008 February 14 with $\mathrm{C}_{2} \mathrm{~S}$ and $\mathrm{NH}_{3}(4,4)$, and all 56 pointings were observed with $\mathrm{H}_{2} \mathrm{O}$ and $\mathrm{NH}_{3}(1,1),(2,2)$, and $(3,3)$. Ten pointings were observed in all six lines (four $\mathrm{NH}_{3}$ lines, $\mathrm{H}_{2} \mathrm{O}$, and $\mathrm{C}_{2} \mathrm{~S}$ ). We observed each source with a symmetric, in-band frequency switch $(5 \mathrm{MHz}$ throw).

Observations of the Gem OB1 region were conducted immediately following observations for a pointing model correction on each night. We calibrated the data with the injection of a noise signal periodically throughout the observations. Because of slow variations in the power output of the noise diodes and their coupling to the signal path, we measured the strength of the noise signal through observations of a source with known flux (the NRAO flux calibrator $3 \mathrm{C} 48, S_{v}=1.13 \mathrm{Jy}$ ). We repeated the flux calibration observations during every observing session to detect any changes in the calibration sources, finding no significant variations over the course of our run $(<10 \%)$. The beam size of the GBT is $31^{\prime \prime}$ at these frequencies, which projects to a size of $0.32 \mathrm{pc}$ at the distance of Gem OB1 $(2.10 \mathrm{kpc}$, see Section 6.1; Reid et al. 2009). The GBT $\mathrm{NH}_{3}$ observations are well matched to the BGPS observations since the GBT beam size is only a few arcseconds smaller than the effective BGPS beam size.

\subsubsection{Data Reduction}

The ammonia observations were reduced using the GBTIDL reduction package. We extracted each scan, using the package to fold the spectrum to remove the effects of the frequency switching and establish the data on the $T_{A}$ scale by calibrating the noise diodes. We subtracted a linear baseline from the spectrum from each IF with the exception of the IF containing the water and $\mathrm{C}_{2} \mathrm{~S}$ lines where a second order baseline was required. We then scaled to the $T_{A}^{*}$ scale using estimates of the atmospheric opacity interpolated in the frequency and time domains at 22 and $23 \mathrm{GHz}$ from models of the atmosphere derived using weather data. ${ }^{17}$ To reach the $T_{\mathrm{mb}}$ scale, the spectra are divided by the main beam efficiency of the GBT, which is $\eta_{\mathrm{mb}} \sim 0.81$ at these frequencies. We averaged together both polarizations; if a source was observed multiple times, each observation was calibrated separately and the resulting spectra were then averaged together. The mean and standard deviation of the rms noise per channel for each of the six observed lines are listed in Table 1.

\section{PARAMETER ESTIMATION}

\subsection{BGPS Parameter Estimation}

The BGPS millimeter source properties are extracted from the iterative maps using the BGPS source extraction software,

\footnotetext{
${ }^{17}$ See http://www.gb.nrao.edu/ rmaddale/Weather.
}

Bolocat (Rosolowsky et al. 2010). Bolocat utilizes a seeded watershed method (also referred to as marker-controlled segmentation; Soille 1999), which is comprised of two main steps. First, regions with emission above a signal-to-noise ratio of 2.5 are identified and expanded through a nearest-neighbor algorithm to include adjacent, lower signal-to-noise pixels. Second, each identified region is subdivided into further substructures based on the contrast between local maxima. Bolocat then determines source properties from emission-weighted moments. Two sets of coordinates are determined for each source: peak and centroid coordinates. The peak coordinates correspond to the peak of the $1.1 \mathrm{~mm}$ emission and are determined from median smoothed maps. The centroid coordinates are given by the first emission-weighted moments. The major and minor axis dispersions $\left(\sigma_{\text {maj }}\right.$ and $\left.\sigma_{\text {min }}\right)$ are given by the second moments. The object radius $\left(R_{\text {obj }}\right)$ is calculated from

$$
R_{\mathrm{obj}}=\eta\left[\left(\sigma_{\mathrm{maj}}^{2}-\sigma_{\text {beam }}^{2}\right)\left(\sigma_{\text {min }}^{2}-\sigma_{\text {beam }}^{2}\right)\right]^{1 / 4},
$$

where $\sigma_{\text {beam }}=\Theta_{\mathrm{FWHM}} / \sqrt{8 \ln 2}$ and $\eta$ is a factor relating the axis dispersions to the true size of the source, found to be 2.4. The value $\eta=2.4$ is adopted as the median value derived from measuring the observed axis dispersions compared with the true radius for a variety of simulated emission profiles spanning a range of density distributions, sizes relative to the beam, and signal-to-noise ratios. The ellipses in Figures 3-5 mark the positions and sizes of the extracted BGPS sources. White ellipses denote BGPS sources which do not contain any $\mathrm{NH}_{3}$ pointings or only contain a pointing which was not detected, and black ellipses denote BGPS sources which contain at least one detected $\mathrm{NH}_{3}$ pointing.

Source flux densities are determined by aperture photometry within standard apertures, $40^{\prime \prime}, 80^{\prime \prime}, 120^{\prime \prime}$, and $2 R_{\text {obj }}$ diameter apertures denoted as $S_{v}\left(40^{\prime \prime}\right), S_{v}\left(80^{\prime \prime}\right), S_{v}\left(120^{\prime \prime}\right)$, and $S_{v}(\mathrm{obj})$. An integrated flux density, $S_{v}$ (int), is also reported for each source and is the sum of all pixels assigned to a given source by the source extraction algorithm divided by the number of pixels per beam. The intensity in the BGPS maps was calibrated based on the observed properties of planets. Aguirre et al. (2010) have compared the BGPS data to other millimeter continuum surveys and found that we must multiply our flux densities by $1.5 \pm 0.15$ in order for our flux densities to agree with the other surveys (see Aguirre et al. 2010 for more details). In this paper, we apply the flux calibration correction of $1.5 \pm 0.15$ to the v1.0 BGPS products presented in Rosolowsky et al. (2010) and released on the IPAC Web site. All data products and properties presented in this paper include this flux calibration factor.

Aperture corrections must be applied to the photometry in order to account for power at large radii due to the non-Gaussian side lobes of the CSO beam. The aperture corrections were determined by comparing the integrated flux density for known point sources to the calculated aperture flux densities. The aperture correction values are listed in Table 2. As can be seen from the aperture corrections, the $40^{\prime \prime}$ aperture underestimates the flux density by almost $50 \%$. The $80^{\prime \prime}$ aperture only underestimates the flux density by approximately $4 \%$, and the $120^{\prime \prime}$ aperture requires only a $1 \%$ correction. The apertures corresponding to $2 R_{\text {obj }}$ require varying aperture corrections, while $S_{v}$ (int) represents all flux in the $1.1 \mathrm{~mm}$ maps down to the $1 \sigma$ level and does not require an aperture correction factor. The aperture corrections were determined based on sources very small compared to the BGPS beam and therefore will slightly overestimate the flux densities of sources which are extended compared to the beam 
Table 2

Aperture Flux Corrections

\begin{tabular}{lc}
\hline \hline Aperture $(")$ & $S_{v}($ int $) / S_{v}(\mathrm{ap})$ \\
\hline 40 & 1.46 \\
80 & 1.04 \\
120 & 1.01 \\
\hline
\end{tabular}

size. For more details regarding source extraction and parameter estimation, see Rosolowsky et al. (2010).

\section{2. $\mathrm{NH}_{3}$ Parameter Estimation}

We estimate physical properties of the molecular gas by fitting a simple model to each calibrated spectrum, following the method described in Rosolowsky et al. (2008, hereafter R08). The model estimates the ammonia emission that would be observed from a slab of molecular gas with a fixed optical depth in the $\mathrm{NH}_{3}(1,1)$ line of $\tau$, local standard of rest (LSR) velocity $v_{\text {LSR }}$, velocity dispersion $\sigma_{v}$, radiative excitation temperature $T_{\mathrm{ex}}$, and kinetic temperature $T_{K}$. For clarity, we note that $\tau$ here represents the total opacity in the $(1,1)$ transition which is then apportioned into the 18 hyperfine components of the line according to their statistical weights. The central cluster of ammonia hyperfine components, which is commonly studied in isolation, contains half the total optical depth, $\tau_{\text {main }}=0.5 \tau$.

The model spectrum provides a simultaneous estimate of all the ammonia parameters; however, specific aspects of the spectral data control each derived quantity. For example, the ratio of strengths of the $(1,1)$ and $(2,2)$ lines yields an estimate of the kinetic temperature. The model accounts for the line widths by assuming a Gaussian velocity distribution in the underlying slab of material so that the model spectrum accounts for line broadening by hyperfine structure and optical depth effects. The difference between the line strength on the $T_{\mathrm{mb}}$ scale and the kinetic temperature provides information on the radiative excitation of the line $T_{\mathrm{ex}}$ under the assumption that the emission fills the telescope beam (see Section 6.6 for more details). As noted in R08, the parameters $T_{\mathrm{ex}}$ and $\tau$ are strongly covariant and cannot be determined separately in the low optical depth limit. In these cases, only the product $\tau T_{\mathrm{ex}}$ is determined. At higher optical depths, the relative strengths of the central and satellite hyperfine transitions in the $(1,1)$ line provide an independent estimate of the optical depth.

Using these parameters as a description of the underlying molecular gas, a model spectrum can be generated that adequately describes emission we observe from the Gem OB1 sources including the hyperfine structure of the ammonia transitions. The best-fit set of parameters is determined by a leastsquares optimization of the model spectrum to fit to all observed ammonia transitions simultaneously. The primary advantage of this approach is the straightforward estimation of errors and covariances in the fit parameters.

The spectral model has two significant differences from that of the R08 study. In particular, the inclusion of $\mathrm{NH}_{3}(3,3)$ lines in the analysis necessitates treating the ortho- and para- $\mathrm{NH}_{3}$ states in more detail. Rather than simply adopting a full thermal equilibrium as done by R08, we treat the two sets of states as different species with an ortho-to-para ratio of $1: 1$, which is the high temperature limit $\left(T_{K}>40 \mathrm{~K}\right.$; Takano et al. 2002). Given that the kinetic temperature derived from only the $(1,1)$ and $(2,2)$ lines provides a good estimate of the $(3,3)$ line in most cases, this assumption is adequate. As such, partition functions for the two species are decoupled and contain only the ortho- and the para-states:

$$
\begin{aligned}
& Z_{O}=1+\sum_{J} 2(2 J+1) \exp \left\{\frac{-h\left[B J(J+1)+(C-B) J^{2}\right]}{k T_{k}}\right\} \\
& \text { for } J=3,6,9, \ldots
\end{aligned}
$$

$$
\begin{aligned}
& Z_{P}=\sum_{J}(2 J+1) \exp \left\{\frac{-h\left[B J(J+1)+(C-B) J^{2}\right]}{k T_{k}}\right\} \\
& \text { for } J=1,2,4,5, \ldots,
\end{aligned}
$$

where we have only included the metastable states given by $J=$ $K$. The lifetimes of the non-metastable states are relatively short and the levels are not well populated. The partition functions are subject to the constraint that $Z_{O}=Z_{P}$, representing our assumption of the 1:1 ortho-to-para ratios. In addition to a different partition function, we also model the effects of the frequency switching explicitly in the model spectrum since the frequency switch is smaller than in the R08 study. We build a frequency switched version of the spectrum by shifting it $\pm 5 \mathrm{MHz}$ in frequency and subtracting half of the amplitude in the model. Thus, we fit both the peaks and the negative features in the observed spectra, resulting in a fit which is more sensitive to the low-intensity emission.

Emission from the $\mathrm{H}_{2} \mathrm{O}$ and $\mathrm{C}_{2} \mathrm{~S}$ lines is not treated in detail by the model but rather by simply calculating its velocity-integrated intensity. Since the model is necessarily simple, complicated sources will be represented by average properties. However, the model produces good fits for the wide variety of sources found in the observations.

\section{RESULTS}

\section{1. $1.1 \mathrm{~mm}$ Results}

We have extracted $55 \mathrm{~mm}$ continuum sources from the BGPS maps. The $1.1 \mathrm{~mm}$ sources are listed in Table 3, along with their ID number in the complete BGPS source catalog, their corresponding group number (see Section 3.1.3), and any coincident IRAS sources, Two Micron All Sky Survey (2MASS) sources, and well-known millimeter sources. Table 4 lists extracted $1.1 \mathrm{~mm}$ source properties including a running source ID number (Column 1), the source name (based on the Galactic coordinates of the peak emission; Column 2), centroid R.A. and decl. (Columns 3 and 4), R.A. and decl. of the peak emission (Columns 5 and 6), size of semimajor and semiminor axes in arcseconds (Columns 7 and 8), position angle of bestfit ellipse measured counterclockwise from the $x$-axis $\left(\ell=0^{\circ}\right)$ in Galactic coordinates (Column 9), the deconvolved source radius $R_{\text {obj }}$ (Column 10), and $S_{v}\left(120^{\prime \prime}\right)$ and $S_{\nu}$ (int) (Columns 11 and 12). Some sources are sufficiently small that a deconvolved radius cannot be determined, and we assume a source diameter equal to the beam FWHM as an upper limit on the radius for these objects.

$S_{v}$ (int) and $R_{\text {obj }}$ adequately characterize the sources but these two quantities are highly dependent upon the source extraction algorithm and are not easily compared with other observations. In order to support comparison of our results with other studies, we will also present a flux within a well-characterized and commonly used aperture.

To evaluate which aperture most closely represents the true millimeter flux, we consider the derived object radii as well 
Table 3

Millimeter Sources

\begin{tabular}{|c|c|c|c|c|}
\hline $\begin{array}{c}\text { ID } \\
\text { Number }\end{array}$ & Source & $\begin{array}{c}\text { BGPS ID } \\
\text { Number }\end{array}$ & $\begin{array}{c}\text { Group } \\
\text { Number }\end{array}$ & Comments \\
\hline 01 & G189.646+00.131 & 7469 & 2 & \\
\hline 02 & G189.659+00.185 & 7470 & 2 & \\
\hline 03 & G189.682+00.185 & 7471 & 2 & IRAS $06047+2040$ \\
\hline 04 & G189.782+00.265 & 7475 & 2 & IRAS $06053+2036$ \\
\hline 05 & G189.116+00.643 & 7467 & 1 & IRAS $06052+2122$ \\
\hline 06 & G189.788+00.281 & 7479 & 2 & \\
\hline 07 & G189.713+00.335 & 7472 & 2 & \\
\hline 08 & G189.789+00.291 & 7480 & 2 & \\
\hline 09 & G189.744+00.335 & 7473 & 2 & \\
\hline 10 & G189.782+00.323 & 7476 & 2 & \\
\hline 11 & G189.836+00.303 & 7485 & 2 & IRAS 06055+2034 \\
\hline 12 & G189.950+00.231 & 7491 & 2 & \\
\hline 13 & G189.776+00.343 & 7474 & 2 & S252A, IRAS 06055+2039 \\
\hline 14 & G189.834+00.317 & 7484 & 2 & \\
\hline 15 & G189.888+00.303 & 7489 & 2 & IRAS 06056+2032 \\
\hline 16 & G189.804+00.355 & 7481 & 2 & \\
\hline 17 & G189.030+00.781 & 7465 & 1 & IRAS 06056+2131 \\
\hline 18 & G189.831+00.343 & 7843 & 2 & J06084309+2036182 \\
\hline 19 & G189.879+00.319 & 7487 & 2 & \\
\hline 20 & G189.885+00.319 & 7488 & 2 & \\
\hline 21 & G189.810+00.369 & 7482 & 2 & \\
\hline 22 & G189.032+00.793 & 7466 & 1 & IRAS 06056+2131 \\
\hline 23 & G189.921+00.331 & 7490 & 2 & \\
\hline 24 & G189.015+00.823 & 7464 & 1 & \\
\hline 25 & G188.991+00.859 & 7463 & 1 & \\
\hline 26 & G188.948+00.883 & 7461 & 1 & S252 \\
\hline 27 & G189.951+00.331 & 7492 & 2 & \\
\hline 28 & G189.783+00.433 & 7477 & 2 & IRAS 06059+2042 \\
\hline 29 & G188.975+00.911 & 7462 & 1 & \\
\hline 30 & G189.990+00.353 & 7493 & 2 & \\
\hline 31 & G189.783+00.465 & 7478 & 2 & \\
\hline 32 & G188.792+01.027 & 7460 & 1 & IRAS 06061+2151 \\
\hline 33 & G190.006+00.361 & 7494 & 2 & IRAS $06061+2028$ \\
\hline 34 & G189.864+00.499 & 7486 & 2 & IRAS $06063+2040$ \\
\hline 35 & G189.231+00.893 & 7468 & 1 & IRAS $06065+2124$ \\
\hline 36 & G190.054+00.533 & 7496 & 2 & IRAS 06068+2030 \\
\hline 37 & G190.044+00.543 & 7495 & 2 & \\
\hline 38 & G190.063+00.679 & 7497 & 2 & \\
\hline 39 & G190.192+00.719 & 7499 & 2 & \\
\hline 40 & G190.171+00.733 & 7498 & 2 & IRAS 06078+2030 \\
\hline 41 & G190.240+00.911 & 7500 & 2 & IRAS 06087+2031 \\
\hline 42 & G192.629-00.157 & 7505 & 3 & \\
\hline 43 & G192.602-00.143 & 7503 & 3 & S256, IRAS 06096+1757 \\
\hline 44 & G192.629-00.123 & 7504 & 3 & \\
\hline 45 & G192.581-00.043 & 7501 & 3 & $\mathrm{~S} 255 \mathrm{~N}$ \\
\hline 46 & G192.662-00.083 & 7507 & 3 & \\
\hline 47 & G192.596-00.051 & 7502 & 3 & S255IR, J06125330+1759215 \\
\hline 48 & G192.644+00.003 & 7506 & 3 & $\mathrm{~S} 258$ \\
\hline 49 & G192.719+00.043 & 7508 & 3 & IRAS 06105+1756 \\
\hline 50 & G192.764+00.101 & 7509 & 3 & \\
\hline 51 & G192.816+00.127 & 7510 & 3 & \\
\hline 52 & G192.968+00.093 & 7511 & 3 & \\
\hline 53 & G193.006+00.115 & 7514 & 3 & \\
\hline 54 & G192.981+00.149 & 7512 & 3 & J06142359+1744469 \\
\hline 55 & G192.985+00.177 & 7513 & 3 & \\
\hline
\end{tabular}

as the distance between all BGPS sources. Figure 6 shows the distribution of derived source radii for the 55 BGPS sources (white histogram) and the unresolved sources where we have assigned the beam size as an upper limit for the source radius (gray histogram). The dotted lines mark the radii of the apertures in which we present photometry in the BGPS catalog. There is a large spread in the object radii, with the largest being almost $100^{\prime \prime}$. Figure 7 shows the distribution of angular separation between the emission maxima of all 55 BGPS sources out to $500^{\prime \prime}$. The dotted lines mark the diameters of our chosen apertures.

To characterize the millimeter flux well, we should choose an aperture size greater than the largest source radius yet small enough to reduce the number of sources which will include flux 
Table 4

Observed $1.1 \mathrm{~mm}$ Properties

\begin{tabular}{|c|c|c|c|c|c|c|c|c|c|c|c|}
\hline $\begin{array}{c}\text { ID } \\
\text { Number } \\
\end{array}$ & Source & $\begin{array}{c}\text { R.A. } \\
(\mathrm{J} 2000)\end{array}$ & $\begin{array}{c}\text { Decl. } \\
(\mathrm{J} 2000)\end{array}$ & $\begin{array}{c}\text { Peak R.A. } \\
(\mathrm{J} 2000)\end{array}$ & $\begin{array}{c}\text { Peak Decl. } \\
(\mathrm{J} 2000)\end{array}$ & $\begin{array}{c}R_{\text {major }} \\
\left(^{\prime \prime}\right)\end{array}$ & $\begin{array}{c}R_{\text {minor }} \\
\left({ }^{\prime \prime}\right)\end{array}$ & $\begin{array}{c}\text { P.A. } \\
\left({ }^{\circ}\right)\end{array}$ & $\begin{array}{l}R_{\mathrm{obj}}{ }^{\mathrm{a}} \\
(\mathrm{pc})\end{array}$ & $\begin{array}{c}S_{v}\left(120^{\prime \prime}\right) \\
(\mathrm{mJy})\end{array}$ & $\begin{array}{c}S_{v} \text { (int) } \\
(\mathrm{mJy})\end{array}$ \\
\hline 01 & G189.646+00.131 & 060730.6 & 204000.6 & 060730.9 & 203946.1 & 33.0 & 19.9 & 74 & 0.50 & $936(250)$ & $988(120)$ \\
\hline 02 & G189.659+00.185 & 060744.4 & 204049.6 & 060744.7 & 204036.6 & 20.5 & 14.1 & 139 & $<0.17$ & $1010(270)$ & $370(91)$ \\
\hline 03 & G189.682+00.185 & 060746.9 & 203939.6 & 060747.5 & 203927.4 & 33.5 & 19.2 & 138 & 0.49 & $1509(300)$ & $1700(150)$ \\
\hline 04 & G189.782+00.265 & 060817.3 & 203637.9 & 060817.9 & 203632.6 & 28.2 & 18.0 & 145 & 0.40 & $1380(300)$ & $1190(140)$ \\
\hline 05 & G189.116+00.643 & 060819.9 & 212226.3 & 060819.8 & 212229.2 & 31.2 & 19.9 & 4 & 0.49 & $1190(320)$ & $1090(130)$ \\
\hline 06 & G189.788+00.281 & 060822.8 & 203638.0 & 060822.2 & 203641.7 & 21.7 & 17.6 & 43 & 0.32 & $1630(330)$ & $1019(140)$ \\
\hline 07 & G189.713+00.335 & 060825.4 & 204204.8 & 060825.1 & 204208.9 & 26.3 & 20.6 & 158 & 0.45 & $1360(300)$ & $1160(140)$ \\
\hline 08 & G189.789+00.291 & 060825.8 & 203704.9 & 060824.7 & 203652.8 & 16.5 & 9.1 & 13 & $<0.17$ & $1430(330)$ & $347(94)$ \\
\hline 09 & G189.744+00.335 & 060829.0 & 204104.7 & 060828.8 & 204034.5 & 28.6 & 20.9 & 72 & 0.48 & $2960(420)$ & $1960(210)$ \\
\hline 10 & G189.782+00.323 & 060829.3 & 203813.1 & 060830.9 & 203813.9 & 28.5 & 22.7 & 38 & 0.51 & $3190(450)$ & $2240(220)$ \\
\hline 11 & G189.836+00.303 & 060830.5 & 203433.3 & 060833.1 & 203449.0 & 34.2 & 23.4 & 142 & 0.59 & $2480(380)$ & $1780(210)$ \\
\hline 12 & G189.950+00.231 & 060831.0 & 202649.9 & 060831.1 & 202644.4 & 34.4 & 22.6 & 17 & 0.58 & $1670(310)$ & $1910(160)$ \\
\hline 13 & G189.776+00.343 & 060834.4 & 203918.2 & 060834.6 & 203907.7 & 38.5 & 26.8 & 151 & 0.70 & $8930(850)$ & $11100(410)$ \\
\hline 14 & G189.834+00.317 & 060835.0 & 203510.5 & 060836.0 & 203519.7 & 23.4 & 17.1 & 32 & 0.33 & $2730(400)$ & $1530(170)$ \\
\hline 15 & G189.888+00.303 & 060838.9 & 203206.5 & 060839.6 & 203205.3 & 20.6 & 13.5 & 165 & $<0.17$ & $1370(290)$ & $537(110)$ \\
\hline 16 & G189.804+00.355 & 060839.7 & 203802.0 & 060840.8 & 203800.5 & 23.4 & 22.2 & 40 & 0.44 & $8060(790)$ & $4700(320)$ \\
\hline 17 & G189.030+00.781 & 060840.2 & 213100.0 & 060840.1 & 213100.8 & 26.9 & 22.8 & 85 & 0.50 & $11100(990)$ & $11200(410)$ \\
\hline 18 & G189.831+00.343 & 060841.3 & 203604.8 & 060841.6 & 203611.4 & 36.2 & 28.9 & 73 & 0.71 & $5780(610)$ & $6420(350)$ \\
\hline 19 & G189.879+00.319 & 060843.2 & 203349.9 & 060842.2 & 203258.4 & 39.2 & 21.9 & 58 & 0.61 & $2390(350)$ & $2400(210)$ \\
\hline 20 & G189.885+00.319 & 060844.5 & 203212.0 & 060842.9 & 203239.5 & 32.5 & 22.7 & 1 & 0.56 & $2450(360)$ & $2080(200)$ \\
\hline 21 & G189.810+00.369 & 060844.6 & 203818.5 & 060844.7 & 203806.1 & 54.4 & 31.8 & 66 & 0.95 & $8090(780)$ & $11300(480)$ \\
\hline 22 & G189.032+00.793 & 060845.7 & 213151.7 & 060843.1 & 213115.4 & 30.9 & 23.7 & 39 & 0.56 & $11100(1000)$ & $6690(430)$ \\
\hline 23 & G189.921+00.331 & 060849.2 & 203146.8 & 060850.1 & 203107.1 & 25.0 & 14.9 & 21 & 0.25 & $2500(380)$ & $697(160)$ \\
\hline 24 & G189.015+00.823 & 060849.7 & 213349.2 & 060847.9 & 213258.1 & 34.9 & 16.2 & 67 & 0.39 & $2690(390)$ & $1660(190)$ \\
\hline 25 & G188.991+00.859 & 060853.1 & 213532.0 & 060853.0 & 213516.5 & 30.2 & 12.6 & 51 & $<0.17$ & 977 (270) & 707 (110) \\
\hline 26 & G188.948+00.883 & 060853.4 & 213823.8 & 060852.9 & 213816.9 & 29.0 & 24.4 & 89 & 0.55 & $12899(1200)$ & $14599(450)$ \\
\hline 27 & G189.951+00.331 & 060853.6 & 202937.3 & 060853.8 & 202932.6 & 53.5 & 34.9 & 157 & 0.99 & $5980(620)$ & $10800(390)$ \\
\hline 28 & G189.783+00.433 & 060855.5 & 204116.0 & 060855.8 & 204119.6 & 43.6 & 22.4 & 44 & 0.66 & $1439(300)$ & $1840(160)$ \\
\hline 29 & G188.975+00.911 & 060903.6 & 213803.8 & 060902.7 & 213737.5 & 26.6 & 16.2 & 56 & 0.33 & $718(260)$ & $703(95)$ \\
\hline 30 & G189.990+00.353 & 060903.7 & 202818.0 & 060903.4 & 202811.3 & 22.3 & 14.0 & 3 & $<0.17$ & $886(280)$ & $499(91)$ \\
\hline 31 & G189.783+00.465 & 060904.4 & 204212.6 & 060903.0 & 204215.4 & 30.0 & 19.8 & 124 & 0.47 & 917 (270) & $949(120)$ \\
\hline 32 & G188.792+01.027 & 060906.7 & 215048.5 & 060906.0 & 215039.1 & 27.2 & 22.2 & 83 & 0.49 & $4240(570)$ & $4650(250)$ \\
\hline 33 & G190.006+00.361 & 060908.4 & 202733.7 & 060907.2 & 202734.9 & 30.5 & 20.1 & 110 & 0.48 & $887(260)$ & $760(110)$ \\
\hline 34 & G189.864+00.499 & 060920.7 & 203930.8 & 060920.6 & 203902.7 & 50.2 & 36.5 & 27 & 0.99 & $3850(450)$ & $6969(300)$ \\
\hline 35 & G189.231+00.893 & 060930.4 & 212344.7 & 060930.6 & 212339.8 & 17.4 & 15.4 & 100 & 0.20 & $464(260)$ & 427 (79) \\
\hline 36 & G190.054+00.533 & 060951.1 & 203014.5 & 060951.7 & 203003.2 & 52.7 & 23.4 & 21 & 0.75 & $2220(340)$ & $2930(220)$ \\
\hline 37 & G190.044+00.543 & 060954.6 & 203056.2 & 060952.7 & 203052.2 & 25.1 & 17.7 & 22 & 0.37 & $2420(360)$ & $1019(170)$ \\
\hline 38 & G190.063+00.679 & 061027.0 & 203348.5 & 061025.7 & 203345.6 & 20.3 & 12.9 & 149 & $<0.17$ & $418(230)$ & $229(64)$ \\
\hline 39 & G190.192+00.719 & 061050.3 & 202751.6 & 061050.5 & 202811.4 & 16.4 & 12.1 & 29 & $<0.17$ & $685(260)$ & $316(74)$ \\
\hline 40 & G190.171+00.733 & 061051.3 & 202957.7 & 061051.2 & 202938.8 & 45.1 & 16.9 & 32 & 0.49 & $1540(300)$ & $2090(160)$ \\
\hline 41 & G190.240+00.911 & 061140.4 & 203126.7 & 061139.6 & 203112.8 & 28.0 & 17.2 & 73 & 0.38 & $726(260)$ & $626(98)$ \\
\hline 42 & G192.629-00.157 & 061233.6 & 175448.7 & 061233.5 & 175440.4 & 21.9 & 16.7 & 57 & 0.30 & $796(290)$ & $558(96)$ \\
\hline 43 & G192.602-00.143 & 061233.6 & 175619.8 & 061233.2 & 175633.1 & 25.9 & 16.8 & 11 & 0.35 & $894(300)$ & $868(110)$ \\
\hline 44 & G192.629-00.123 & 061239.8 & 175528.8 & 061241.0 & 175539.2 & 35.6 & 21.3 & 16 & 0.56 & 709 (290) & $740(110)$ \\
\hline 45 & G192.581-00.043 & 061252.8 & 180032.6 & 061252.9 & 180029.0 & 25.2 & 21.9 & 174 & 0.46 & $14600(1300)$ & $11400(450)$ \\
\hline 46 & G192.662-00.083 & 061253.1 & 175521.0 & 061253.7 & 175507.3 & 33.5 & 11.7 & 43 & $<0.17$ & $565(310)$ & $463(85)$ \\
\hline 47 & G192.596-00.051 & 061254.1 & 175854.1 & 061252.8 & 175931.0 & 46.5 & 29.5 & 22 & 0.83 & $15400(1400)$ & $17300(590)$ \\
\hline 48 & G192.644+00.003 & 061309.8 & 175840.3 & 061310.6 & 175832.6 & 28.1 & 22.8 & 45 & 0.51 & $1330(320)$ & $1380(140)$ \\
\hline 49 & G192.719+00.043 & 061328.0 & 175602.1 & 061328.6 & 175541.6 & 43.2 & 27.3 & 120 & 0.76 & $2320(390)$ & $3580(220)$ \\
\hline 50 & G192.764+00.101 & 061346.7 & 175500.4 & 061346.8 & 175502.5 & 39.6 & 21.0 & 146 & 0.59 & $2020(370)$ & $2480(180)$ \\
\hline 51 & G192.816+00.127 & 061358.8 & 175251.2 & 061358.8 & 175303.0 & 20.1 & 13.2 & 39 & $<0.17$ & $615(320)$ & $505(84)$ \\
\hline 52 & G192.968+00.093 & 061408.3 & 174404.6 & 061409.6 & 174403.9 & 25.4 & 16.2 & 166 & 0.32 & $682(310)$ & $620(93)$ \\
\hline 53 & G193.006+00.115 & 061418.8 & 174236.9 & 061419.1 & 174241.7 & 19.4 & 14.8 & 162 & 0.19 & $1310(350)$ & $661(110)$ \\
\hline 54 & G192.981+00.149 & 061423.4 & 174428.4 & 061423.7 & 174456.1 & 49.6 & 25.5 & 43 & 0.78 & $4290(520)$ & $6480(290)$ \\
\hline 55 & G192.985+00.177 & 061430.2 & 174534.0 & 061430.4 & 174531.6 & 18.4 & 14.3 & 85 & $<0.17$ & $844(320)$ & $516(90)$ \\
\hline
\end{tabular}

Notes. Errors are given in parentheses.

a Sources that are unresolved with the BGPS beam are assigned the beam size as an upper limit on the object radius.

from their neighbors. If we choose an aperture much larger than the source and no other millimeter sources fall within that aperture, then the flux will still be representative of the total millimeter flux since the background averages to zero. The distribution of source sizes supports the use of the $120^{\prime \prime}$ aperture, which is only smaller than 10 of the 55 BGPS sources. Figure 7 shows that for the $120^{\prime \prime}$ aperture, only approximately five source fluxes will be contaminated by emission from nearby sources.

We have chosen to present the $S_{v}\left(120^{\prime \prime}\right)$ fluxes as wellcharacterized fluxes for comparison with other studies. We 


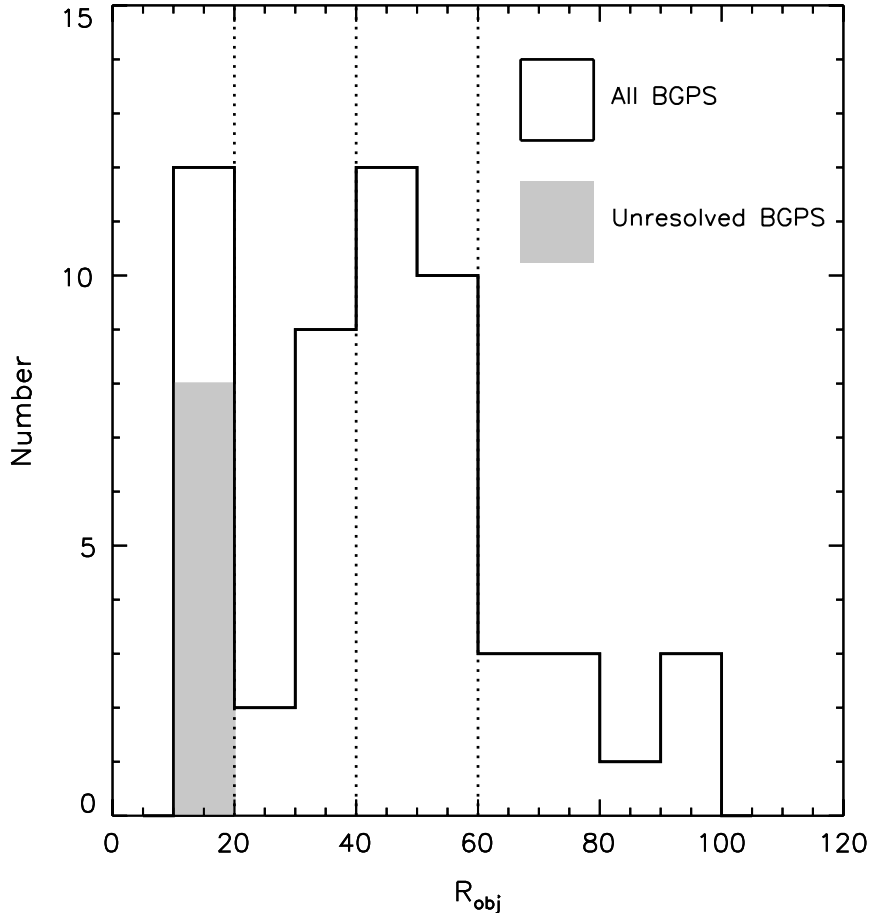

Figure 6. Distribution of extracted source radii for all 55 BGPS sources. The white histogram marks all 55 sources while the gray histogram marks the unresolved sources for which we have set the radius to an upper limit of the beam size. The dotted lines mark the radii of the aperture sizes used in the BGPS catalog, $R_{\text {ap }}=20^{\prime \prime}, 40^{\prime \prime}$, and $60^{\prime \prime}$.

present masses calculated from both $S_{v}\left(120^{\prime \prime}\right)$ and $S_{v}$ (int). However, when calculating physical properties which are highly dependent upon size, such as the volume and surface densities, we will present $S_{\nu}$ (int) and use the derived source radius as the physical size.

\section{2. $\mathrm{NH}_{3}$ Results}

Example spectra of the $\mathrm{NH}_{3}(1,1),(2,2),(3,3)$, and $\mathrm{H}_{2} \mathrm{O}$ lines are shown in Figure 8(a) for the source G189.776+00.343 (ID No. 13). Spectra for all $\mathrm{NH}_{3}$ pointings are available as a figure set in the online edition of the journal. $\mathrm{NH}_{3}(4,4)$ and $\mathrm{C}_{2} \mathrm{~S}$ were not observed for many sources and are not shown here. We detected a $>5 \sigma \mathrm{NH}_{3}(1,1)$ line in $95 \%$ (53 of 56) of all $\mathrm{NH}_{3}$ pointings, and $60 \%$ (33) of the pointings had at least a $5 \sigma$ detection in both the $(1,1)$ and $(2,2)$ lines, where a $5 \sigma$ detection means the integrated intensity is greater than five times the measured error in the integrated intensity for each source.

\subsection{Combined $1.1 \mathrm{~mm}$ and $\mathrm{NH}_{3}$ Results}

Each $\mathrm{NH}_{3}$ pointing was assigned to a single distinct BGPS source. Of the 55 BPGS sources, 37 have ammonia observations along a line of sight that intersects the source. Three BGPS sources have an ammonia pointing associated with the source that lacks any discernible $\mathrm{NH}_{3}(1,1)$ emission. In the remaining 34 BGPS sources, there may be multiple $\mathrm{NH}_{3}$ pointings within the contours of the BGPS source, and the $\mathrm{NH}_{3}$ pointings do not necessarily correspond to either the centroid or peak coordinates of each BGPS source because they were chosen by eye prior to completion of the final BGPS maps. We exclude the seven $\mathrm{NH}_{3}$ pointings that do not fall within the contours of an extracted BGPS source from the analysis. These pointings correspond to low significance BGPS sources. The seven pointings all have

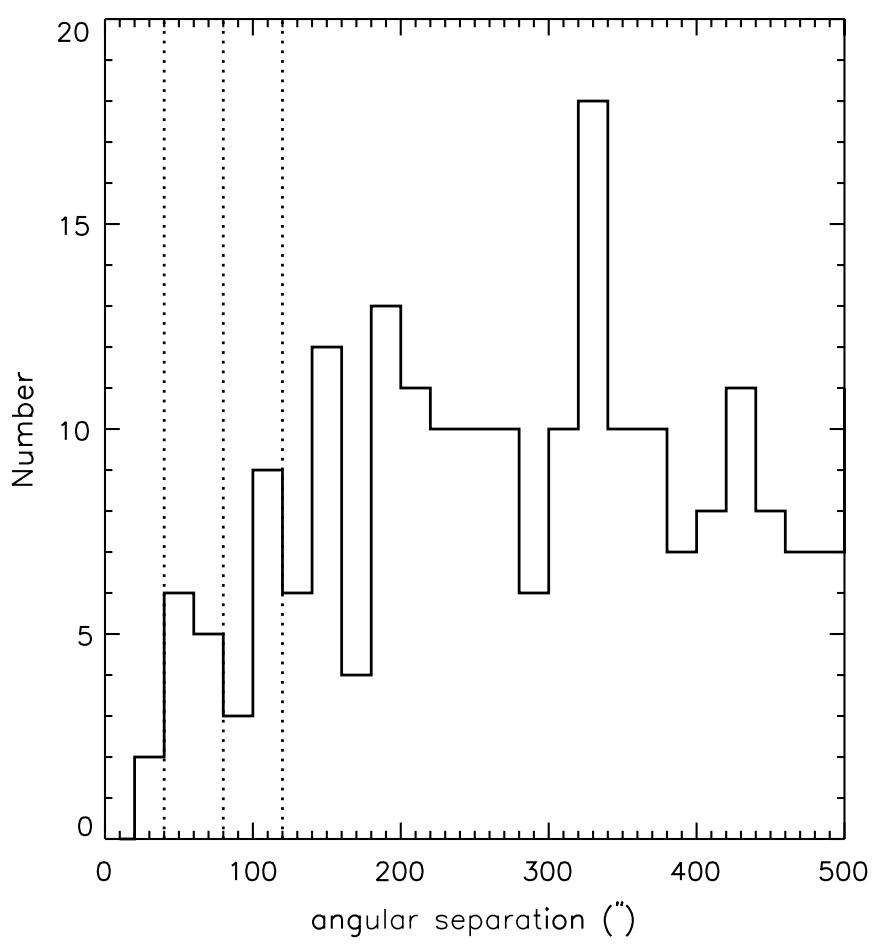

Figure 7. Distribution of distances between the 55 BGPS sources out to 500". The dotted lines mark the diameters of the apertures used in the BGPS catalog, $\theta_{\text {ap }}=40^{\prime \prime}, 80^{\prime \prime}$, and $120^{\prime \prime}$. Only approximately five sources will suffer from contamination with the $120^{\prime \prime}$ aperture.

significant $\mathrm{NH}_{3}(1,1)$ emission, indicating that many marginal detections in the BGPS not included in the catalog are likely real.

In this analysis, we will consider only the $\mathrm{NH}_{3}$ pointing which is located closest to the peak of the millimeter emission. The angular separation between the closest $\mathrm{NH}_{3}$ pointing and the peak of the millimeter emission is shown in Figure 9. The white histogram includes all $\mathrm{NH}_{3}$ pointings while the striped histogram includes only the $\mathrm{NH}_{3}$ pointings closest to the millimeter peak. Most of the $\mathrm{NH}_{3}$ pointings assigned to a BGPS source are located less than an FWHM beam away from the millimeter peak.

We define a "full sample" which consists of 34 BGPS sources that have at least a $5 \sigma$ detection in the $\mathrm{NH}_{3}(1,1)$ transition. Sources without a $5 \sigma$ detection in the $\mathrm{NH}_{3}(2,2)$ transition can only provide an upper limit to the derived gas properties such as $T_{K}$. Consequently, the full sample can only characterize upper limits for these properties. We also define "subset 1 " which is comprised of 25 BGPS sources with a $>5 \sigma$ detection in both the $\mathrm{NH}_{3}(1,1)$ and $(2,2)$ transitions. However, subset 1 may not be characteristic of the faint end of the full sample. Due to the complementary properties of each sample, we will present the characteristics of both. For clarity, we point out that subset 1 is entirely contained in the full sample.

Observed properties of $\mathrm{NH}_{3}$ pointings in the full sample are given in Table 5, including the source ID (Column 1), specific coordinates of the $\mathrm{NH}_{3}$ pointing which do not necessarily correspond to the peak of the millimeter emission (Columns 2 and 3), radial velocity and Gaussian $1 / e$ line width (Columns 4 and 5), and main beam temperature and integrated intensities for the $\mathrm{NH}_{3}(1,1),(2,2),(3,3)$, and $(4,4)$ transitions (Columns 6-13). For undetected lines, we present upper limits for the main beam temperature and integrated intensities given as $T_{\mathrm{mb}}<5 \mathrm{rms}$ and $W<5 \mathrm{rms} \Delta v / \sqrt{(} N)$, where rms is the noise per channel of 

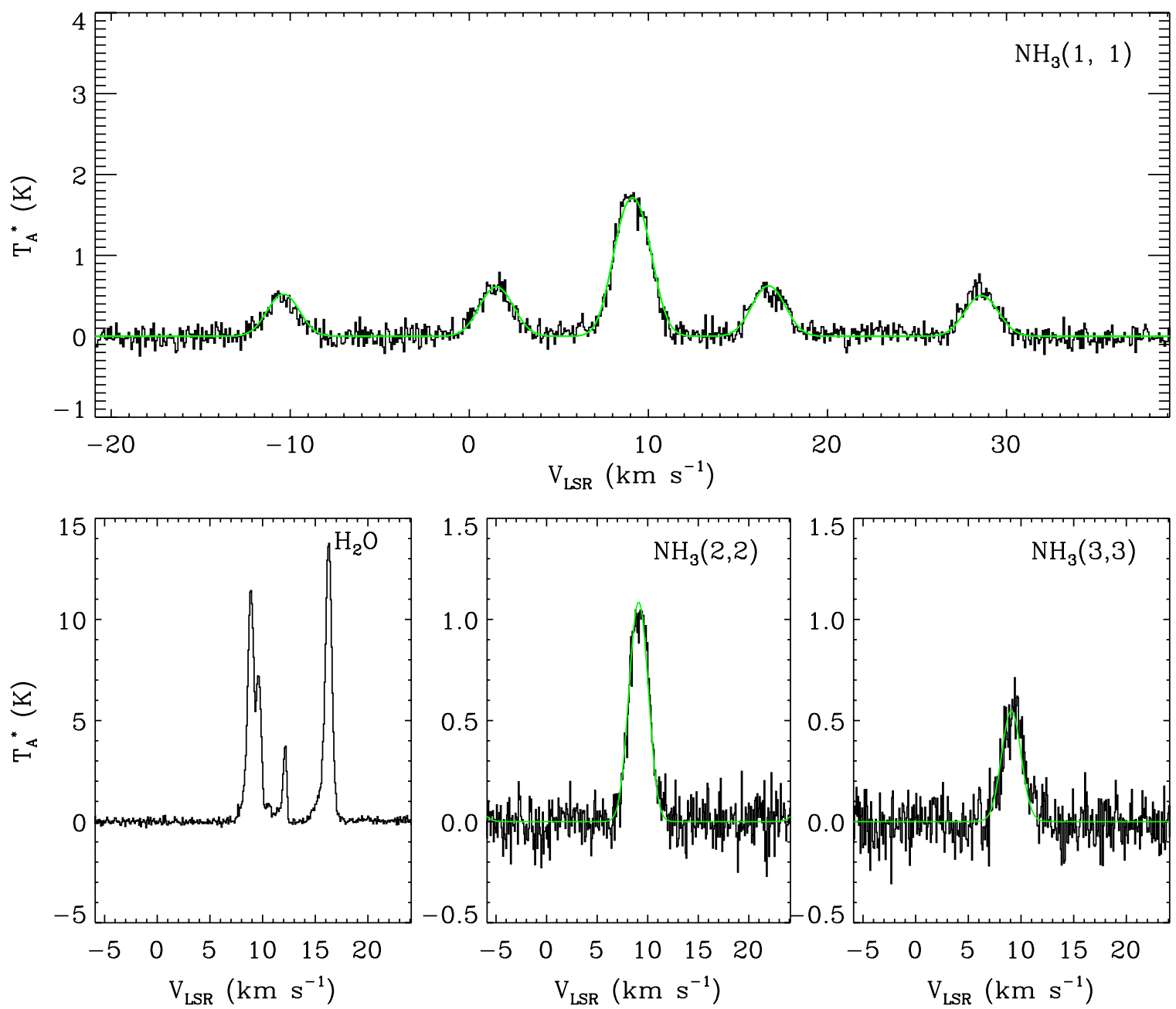

Figure 8. $\mathrm{H}_{2} \mathrm{O}, \mathrm{NH}_{3}(1,1),(2,2)$, and $(3,3)$ spectra for G189.776+0.343 (ID No. 13). Top panel: black is the observed $\mathrm{NH}_{3}(1,1)$ spectrum and green is the model fit. From left to right, the bottom panels show the $\mathrm{H}_{2} \mathrm{O}, \mathrm{NH}_{3}(2,2)$, and $\mathrm{NH}_{3}(3,3)$ spectra.

(A color version of this figure and the complete figure set (46 images) are available in the online journal.)

each spectrum, $\Delta v$ is the channel width in velocity, and $N$ is the number of channels used to calculate the rms.

Table 6 lists derived gas properties including the measured optical depth of the $(1,1)$ transition (Column 2), kinetic gas temperature (Column 3; see Section 6.2), excitation temperature of the $\mathrm{NH}_{3}(1,1)$ transition (Column 4), derived sound speed and non-thermal velocity dispersion ( $a$ and $\sigma_{\mathrm{NT}}$; Columns 5 and 6; see Section 6.3), and flags for detection or non-detection of the $\mathrm{H}_{2} \mathrm{O}$ and CCS lines (Columns 7 and 8). The additional $12 \mathrm{NH}_{3}$ pointings are included in Tables 5 and 6, but are not included in the general analysis. Numbers given in parentheses are errors in the same units as each column.

The distributions of the flux densities, $S_{v}\left(120^{\prime \prime}\right)$ and $S_{\nu}$ (int), are shown in Figure 10. The white histogram includes all 55 BGPS sources extracted from the millimeter maps. The gray histogram includes all BGPS sources included in the full sample $\left(>5 \sigma\right.$ detection in $\mathrm{NH}_{3}(1,1)$ ), while the striped histogram denotes subset 1 ( $>5 \sigma$ detection in $\mathrm{NH}_{3}(1,1)$ and $(2,2))$. The faintest sources are excluded from subset 1 , as expected.

Figure 11 plots the peak $T_{\mathrm{mb}}$ of the $(2,2)$ transition versus the peak $T_{\mathrm{mb}}$ of the $(1,1)$ transition. Pointings with $<5 \sigma$ detection in the $(2,2)$ transition are shown as upper limits. The fainter lines in $(1,1)$ are typically the pointings with a weak or non-detection of $(2,2)$. The weaker BGPS sources typically have weaker
$\mathrm{NH}_{3}(1,1)$ detections and only upper limits for the $\mathrm{NH}_{3}(2,2)$ line.

\subsection{Maser Activity}

We also obtained observations of the $22 \mathrm{GHz}$ water maser in conjunction with our GBT $\mathrm{NH}_{3}$ observations. Eight of the 34 BGPS sources presented here have water maser emission (see Columns 7 and 8 of Table 6). Of these, only source Nos. 16 and 24 have not been reported previously as maser sources. The remaining six BGPS sources with maser activity were previously known to exhibit water as well as some $\mathrm{SiO}$ or methanol maser activity in a $1^{\prime}$ region around the observed GBT position based on a SIMBAD search. The fraction, $8 / 34=0.2$, can be regarded as a lower limit as two sources, Nos. 13 and 47 , show maser emission along one line of sight but not another line of sight through the same core. Since the GBT ammonia spectroscopy was not complete mapping, some sources may have maser emission elsewhere in the object. However, the masing lines of sight are those nearest the peaks of the BGPS emission, so the lower limit is likely representative of the true fraction. For comparison, Szymczak et al. (2005) find a detection fraction of water masers of 0.52 using $6.7 \mathrm{GHz}$ methanol masers, associated with massive YSOs, as a prior. Similarly, Chambers et al. (2009) find detection rates of $0.16,0.54$, and 0.59 for water masers in their IRDC cores classified with 


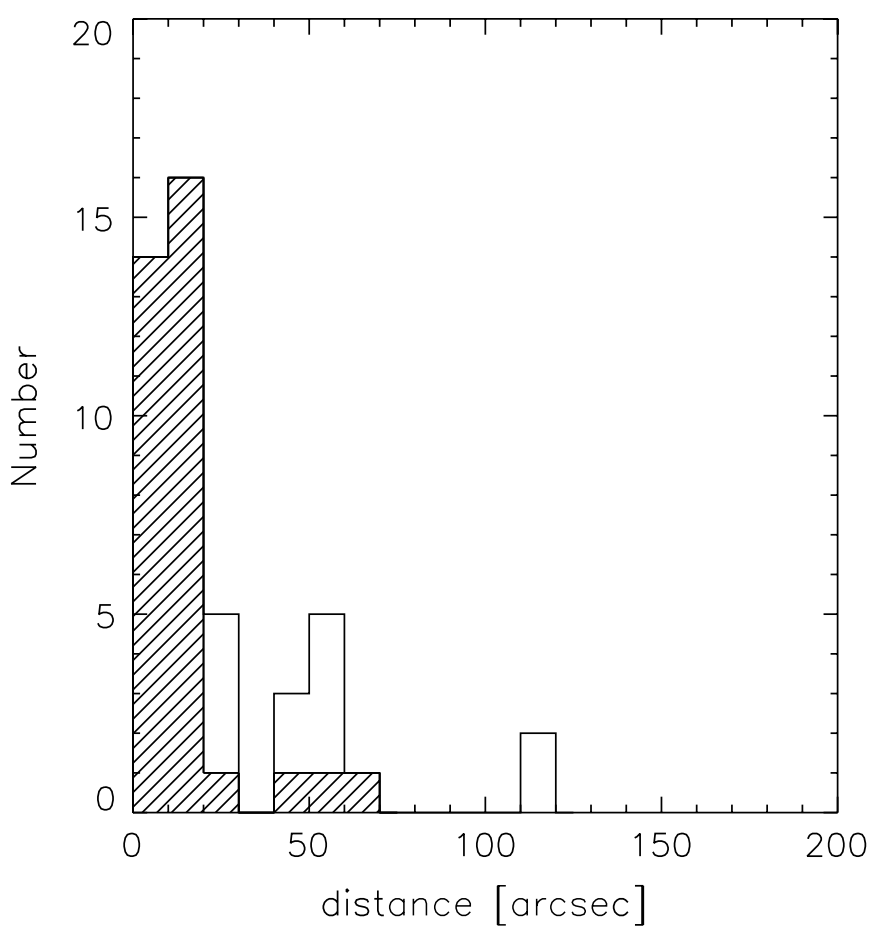

Figure 9. White histogram denotes the distance between each of the 46 crossmatched $\mathrm{NH}_{3}$ pointings and the peak of the associated BGPS source. The striped histogram marks the distance between the peak in $1.1 \mathrm{~mm}$ emission and the nearest $\mathrm{NH}_{3}$ pointing for the 34 BGPS sources in the full sample. The $\mathrm{NH}_{3}$ pointings were chosen by eye from preliminary BGPS images, which can account for the offsets larger than the beam size in the striped histogram. The large distances in the white histogram result from multiple $\mathrm{NH}_{3}$ pointings per BGPS source, with typical spacings between pointings on order of the GBT beam size ( $\left.31^{\prime \prime}\right)$ or larger.

Spitzer data as "quiescent," "red" and "active," respectively. The BGPS-selected water maser detection fraction is most similar to the detection fraction in "quiescent" IRDCs determined by Chambers et al. (2009). We see no evidence for maser activity in the $\mathrm{NH}_{3}(3,3)$ transition. All $(3,3)$ spectra are consistent with the

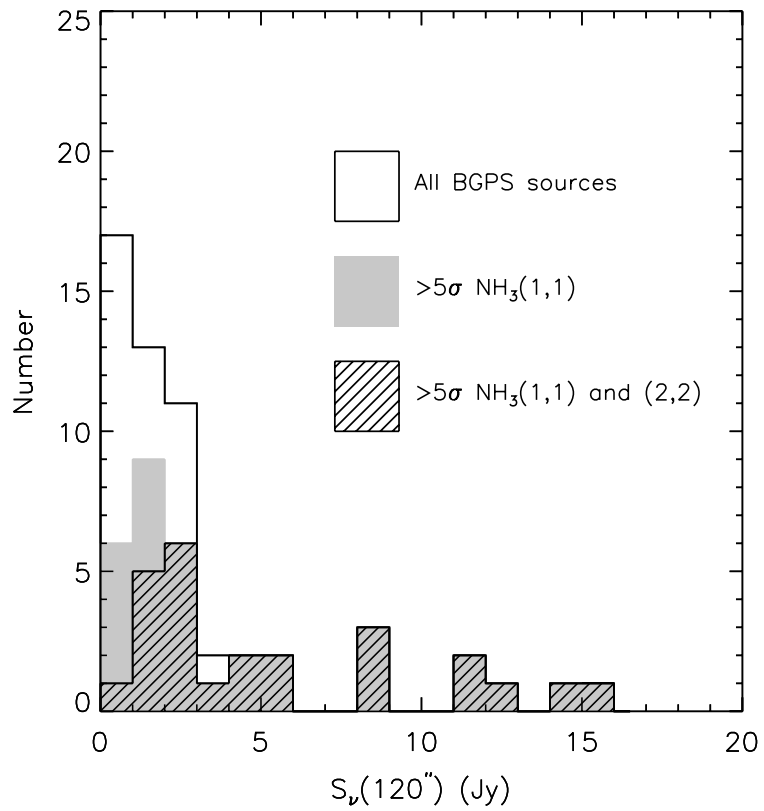

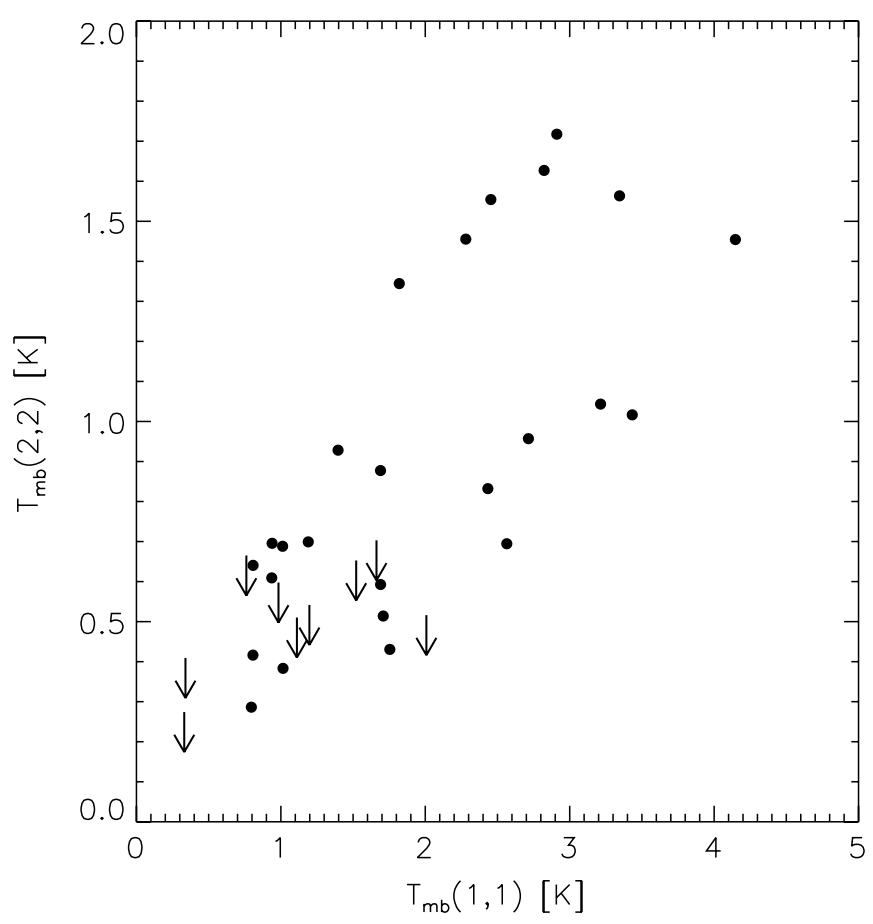

Figure 11. Peak main beam temperature of the $\mathrm{NH}_{3}(1,1)$ line vs. the $(2,2)$ line. Sources in subset 1 are marked by solid circles. Sources excluded from subset 1 give only upper limits for the $(2,2)$ transition and are marked by arrows.

temperatures and line widths determined in conjunction with the $(1,1)$ and $(2,2)$ states. We defer further discussion of the water maser detection fraction to a later paper (M. K. Dunham et al. 2010, in preparation).

\section{ANALYSIS}

\subsection{Radial Velocities and Distances}

We adopt the VLBI parallax distance to S252 of $2.10 \pm$ $0.026 \mathrm{kpc}$ (Reid et al. 2009) for the entire sample of BGPS

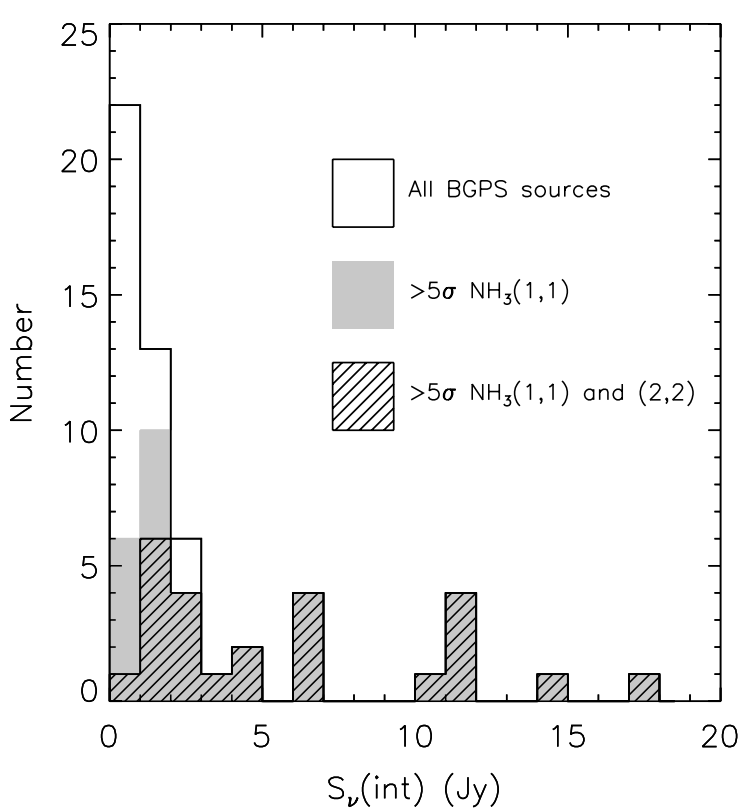

Figure 10. Distribution of $S_{v}\left(120^{\prime \prime}\right)$ (left) and integrated flux densities, $S_{v}$ (int) (right). The white histograms include all extracted $1 \mathrm{~mm}$ sources, the gray histograms represent the full sample (all sources with a $>5 \sigma$ detection in $\mathrm{NH}_{3}(1,1)$ ), and the striped histograms represent subset 1 (all sources with a $>5 \sigma$ detection in $\mathrm{NH}_{3}(1,1)$ and $(2,2))$. 
Table 5

Observed $\mathrm{NH}_{3}$ Properties

\begin{tabular}{|c|c|c|c|c|c|c|c|c|c|c|c|c|}
\hline $\begin{array}{c}\text { ID } \\
\text { Number }^{\mathrm{a}}\end{array}$ & $\begin{array}{l}\text { R.A. } \\
\text { (J2000) }\end{array}$ & $\begin{array}{l}\text { Decl. } \\
\text { (J2000) }\end{array}$ & $\begin{array}{c}\begin{array}{c}V_{\mathrm{LSR}} \\
\left(\mathrm{km} \mathrm{s}^{-1}\right)\end{array} \\
\end{array}$ & $\begin{array}{c}\sigma(1,1) \\
\left(\mathrm{km} \mathrm{s}^{-1}\right)\end{array}$ & $\begin{array}{c}T_{\mathrm{MB}}(1,1) \\
(\mathrm{K})\end{array}$ & $\begin{array}{c}W(1,1) \\
\left(\mathrm{K} \mathrm{km} \mathrm{s}^{-1}\right)\end{array}$ & $\begin{array}{c}T_{\mathrm{MB}}(2,2)^{\mathrm{b}} \\
(\mathrm{K})\end{array}$ & $\begin{array}{c}W(2,2)^{\mathrm{b}, \mathrm{c}} \\
\left(\mathrm{K} \mathrm{km} \mathrm{s}^{-1}\right)\end{array}$ & $\begin{array}{c}T_{\mathrm{MB}}(3,3)^{\mathrm{b}, \mathrm{c}} \\
(\mathrm{K})\end{array}$ & $\begin{array}{c}W(3,3)^{\mathrm{b}, \mathrm{c}} \\
\left(\mathrm{K} \mathrm{km} \mathrm{s}^{-1}\right)\end{array}$ & $\begin{array}{c}T_{\mathrm{MB}}(4,4)^{\mathrm{b}, \mathrm{c}} \\
(\mathrm{K})\end{array}$ & $\begin{array}{c}W(4,4)^{\mathrm{b}, \mathrm{c}} \\
\left(\mathrm{K} \mathrm{km} \mathrm{s}^{-1}\right)\end{array}$ \\
\hline 01 & 060730.8 & 203946.1 & $8.60(0.05)$ & $0.72(0.06)$ & $0.76(0.16)$ & $2.39(0.22)$ & $<0.79$ & $<0.44$ & $<0.86$ & $<0.48$ & & $\cdots$ \\
\hline 03 & 060747.7 & 203931.5 & $7.43(0.02)$ & $0.58(0.02)$ & $1.69(0.15)$ & $5.06(0.21)$ & $0.59(0.15)$ & $0.59(0.08)$ & $<0.81$ & $<0.45$ & $\theta$ & $\ldots$ \\
\hline 04 & 060818.2 & 203636.1 & $10.17(0.02)$ & $0.49(0.03)$ & $1.20(0.16)$ & $3.18(0.22)$ & $<0.80$ & $<0.45$ & $<0.83$ & $<0.46$ & $\ldots$ & $\ldots$ \\
\hline 05 & 060819.8 & 212210.4 & $2.48(0.02)$ & $0.49(0.02)$ & $1.66(0.16)$ & $4.89(0.22)$ & $<0.83$ & $<0.46$ & $<0.85$ & $<0.47$ & $\ldots$ & $\ldots$ \\
\hline 06 & 060823.5 & 203632.9 & $10.12(0.02)$ & $0.51(0.02)$ & $0.80(0.08)$ & $2.39(0.11)$ & $0.29(0.08)$ & $0.31(0.04)$ & $<0.84$ & $<0.46$ & $<0.44$ & $<0.24$ \\
\hline 07 & 060825.3 & 204203.3 & $8.69(0.01)$ & $0.48(0.01)$ & $1.75(0.08)$ & $5.15(0.12)$ & $0.43(0.08)$ & $0.47(0.05)$ & $<0.87$ & $<0.48$ & $<0.44$ & $<0.24$ \\
\hline 11 & 060833.3 & 203438.7 & $8.18(0.05)$ & $1.13(0.05)$ & $1.01(0.16)$ & $4.43(0.22)$ & $0.69(0.16)$ & $0.70(0.09)$ & $<0.80$ & $<0.44$ & $\ldots$ & $\ldots$ \\
\hline 12 & 060831.0 & 202644.5 & $5.49(0.01)$ & $0.42(0.01)$ & $2.56(0.16)$ & $8.38(0.22)$ & $0.69(0.15)$ & $0.72(0.08)$ & $<0.78$ & $<0.43$ & $\ldots$ & . \\
\hline 13 & 060835.2 & 203905.6 & $9.11(0.01)$ & $0.87(0.01)$ & $2.91(0.16)$ & $16.27(0.22)$ & $1.72(0.15)$ & $4.06(0.08)$ & $1.17(0.16)$ & $2.09(0.09)$ & $\ldots$ & .. \\
\hline B & 060829.3 & 204028.5 & $7.82(0.02)$ & $0.54(0.03)$ & $1.17(0.15)$ & $3.78(0.21)$ & $0.66(0.15)$ & $0.83(0.08)$ & $<0.79$ & $<0.44$ & $\ldots$ & .. \\
\hline 16 & 060840.6 & 203756.9 & $7.48(0.01)$ & $0.78(0.01)$ & $4.15(0.16)$ & $18.21(0.22)$ & $1.45(0.15)$ & $2.67(0.08)$ & $0.63(0.16)$ & $0.93(0.09)$ & $\ldots$ & .. \\
\hline 17 & 060840.4 & 213103.1 & $2.60(0.01)$ & $0.92(0.02)$ & $2.45(0.16)$ & $11.35(0.22)$ & $1.55(0.16)$ & $2.82(0.09)$ & $0.72(0.16)$ & $1.33(0.09)$ & $\ldots$ & . \\
\hline 18 & 060842.1 & 203619.7 & $7.69(0.01)$ & $0.71(0.01)$ & $2.71(0.15)$ & $11.60(0.21)$ & $0.96(0.15)$ & $1.52(0.08)$ & $<0.77$ & $<0.43$ & $\ldots$ & $\ldots$ \\
\hline 19 & 060843.4 & 203359.7 & $8.44(0.02)$ & $0.91(0.03)$ & $0.81(0.08)$ & $3.52(0.11)$ & $0.42(0.08)$ & $0.57(0.04)$ & $<0.84$ & $<0.46$ & $<0.43$ & $<0.24$ \\
\hline 20 & 060843.9 & 203229.3 & $8.36(0.02)$ & $0.76(0.02)$ & $1.01(0.08)$ & $3.81(0.12)$ & $0.38(0.08)$ & $0.57(0.05)$ & $<0.82$ & $<0.45$ & $0.37(0.09)$ & $0.25(0.05)$ \\
\hline 21 & 060844.4 & 203753.1 & $8.61(0.01)$ & $1.01(0.01)$ & $2.43(0.09)$ & $12.30(0.12)$ & $0.83(0.08)$ & $1.56(0.05)$ & $<0.87$ & $<0.48$ & $<0.43$ & $<0.24$ \\
\hline B & 060847.0 & 203836.4 & $7.51(0.01)$ & $0.55(0.01)$ & $3.89(0.17)$ & $14.04(0.23)$ & $1.29(0.17)$ & $1.85(0.09)$ & $0.71(0.17)$ & $0.54(0.09)$ & $\ldots$ & $\ldots$ \\
\hline 22 & 060845.9 & 213149.5 & $1.89(0.01)$ & $0.58(0.01)$ & $3.35(0.17)$ & $12.13(0.23)$ & $1.56(0.16)$ & $2.21(0.09)$ & $0.64(0.17)$ & $0.71(0.09)$ & . & $\ldots$ \\
\hline 24 & 060849.5 & 213341.4 & $2.66(0.01)$ & $0.38(0.01)$ & $1.71(0.08)$ & $4.25(0.11)$ & $0.51(0.08)$ & $0.46(0.04)$ & $<0.87$ & $<0.48$ & $0.32(0.08)$ & $0.24(0.05)$ \\
\hline 26 & 060853.6 & 213833.8 & $3.03(0.01)$ & $0.93(0.01)$ & $2.82(0.09)$ & $14.09(0.12)$ & $1.63(0.08)$ & $3.65(0.05)$ & $1.30(0.17)$ & $2.37(0.10)$ & $0.36(0.09)$ & $0.45(0.05)$ \\
\hline B & 060852.4 & 213757.2 & $3.51(0.02)$ & $1.30(0.02)$ & $1.75(0.17)$ & $11.36(0.23)$ & $1.13(0.16)$ & $2.84(0.09)$ & $1.02(0.17)$ & $2.04(0.10)$ & $\ldots$ & $\ldots$ \\
\hline 27 & 060853.6 & 202925.5 & $8.35(0.01)$ & $0.58(0.01)$ & $3.21(0.16)$ & $12.88(0.22)$ & $1.04(0.15)$ & $1.56(0.08)$ & $<0.79$ & $<0.44$ & . & $\ldots$ \\
\hline 28 & 060855.4 & 204050.4 & $7.82(0.03)$ & $0.55(0.03)$ & $1.11(0.16)$ & $2.82(0.22)$ & $<0.76$ & $<0.42$ & $<0.79$ & $<0.43$ & $\ldots$ & $\ldots$ \\
\hline 31 & 060903.9 & 204214.7 & $9.39(0.03)$ & $0.39(0.03)$ & $0.33(0.06)$ & $0.88(0.09)$ & $<0.32$ & $<0.18$ & $<0.49$ & $<0.27$ & $<0.43$ & $<0.24$ \\
\hline 32 & 060906.4 & 215041.0 & $-0.53(0.01)$ & $1.27(0.02)$ & $1.40(0.08)$ & $8.47(0.12)$ & $0.93(0.08)$ & $2.24(0.05)$ & $0.82(0.16)$ & $1.54(0.09)$ & $<0.43$ & $<0.24$ \\
\hline 34 & 060920.8 & 203900.6 & $9.34(0.04)$ & $0.64(0.05)$ & $0.81(0.14)$ & $2.08(0.20)$ & $0.64(0.15)$ & $0.62(0.08)$ & $<0.77$ & $<0.43$ & $\ldots$ & $\ldots$ \\
\hline 35 & 060930.7 & 212335.3 & $-1.00(0.05)$ & $0.44(0.06)$ & $0.34(0.08)$ & $0.59(0.12)$ & $<0.44$ & $<0.25$ & $<0.47$ & $<0.26$ & 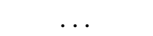 & $\ldots$ \\
\hline 40 & 061051.9 & 202933.7 & $7.83(0.04)$ & $0.81(0.04)$ & $0.94(0.16)$ & $3.61(0.22)$ & $0.61(0.16)$ & $0.46(0.09)$ & $<0.85$ & $<0.47$ & & $\cdots$ \\
\hline B & 061051.8 & 203034.5 & $8.79(0.02)$ & $0.52(0.03)$ & $1.29(0.16)$ & $2.87(0.22)$ & $<0.79$ & $<0.44$ & $<0.82$ & $<0.46$ & $\cdots$ & $\cdots$ \\
\hline 42 & 061234.1 & 175443.3 & $7.71(0.02)$ & $0.50(0.03)$ & $1.19(0.15)$ & $3.04(0.21)$ & $0.70(0.15)$ & $0.63(0.08)$ & $<0.79$ & $<0.44$ & $\cdots$ & $\cdots$ \\
\hline 45 & 061253.3 & 180014.3 & $8.66(0.02)$ & $1.31(0.02)$ & $2.28(0.16)$ & $13.90(0.22)$ & $1.46(0.15)$ & $3.94(0.09)$ & $1.23(0.16)$ & $2.62(0.09)$ & $\cdots$ & $\cdots$ \\
\hline 47 & 061254.1 & 175931.1 & $7.20(0.02)$ & $1.07(0.02)$ & $1.82(0.16)$ & $8.87(0.22)$ & $1.34(0.16)$ & $2.83(0.09)$ & $1.04(0.16)$ & $2.29(0.09)$ & $\ldots$ & $\ldots$ \\
\hline
\end{tabular}


Table 5

Continued)

\begin{tabular}{|c|c|c|c|c|c|c|c|c|c|c|c|c|}
\hline $\begin{array}{c}\text { ID } \\
\text { Number }^{\mathrm{a}}\end{array}$ & $\begin{array}{l}\text { R.A. } \\
\text { (J2000) }\end{array}$ & $\begin{array}{l}\text { Decl. } \\
(\mathrm{J} 2000)\end{array}$ & $\begin{array}{c}V_{\mathrm{LSR}} \\
\left(\mathrm{km} \mathrm{s}^{-1}\right)\end{array}$ & $\begin{array}{c}\sigma(1,1) \\
\left(\mathrm{km} \mathrm{s}^{-1}\right)\end{array}$ & $\begin{array}{c}T_{\mathrm{MB}}(1,1) \\
(\mathrm{K})\end{array}$ & $\begin{array}{c}W(1,1) \\
\left(\mathrm{K} \mathrm{km} \mathrm{s}^{-1}\right)\end{array}$ & $\begin{array}{c}T_{\mathrm{MB}}(2,2)^{\mathrm{b}} \\
(\mathrm{K})\end{array}$ & $\begin{array}{c}W(2,2)^{\mathrm{b}, \mathrm{c}} \\
\left(\mathrm{K} \mathrm{km} \mathrm{s}^{-1}\right)\end{array}$ & $\begin{array}{c}T_{\mathrm{MB}}(3,3)^{\mathrm{b}, \mathrm{c}} \\
(\mathrm{K})\end{array}$ & $\begin{array}{c}W(3,3)^{\mathrm{b}, \mathrm{c}} \\
\left(\mathrm{K} \mathrm{km} \mathrm{s}^{-1}\right)\end{array}$ & $\begin{array}{c}T_{\mathrm{MB}}(4,4)^{\mathrm{b}, \mathrm{c}} \\
(\mathrm{K})\end{array}$ & $\begin{array}{c}W(4,4)^{\mathrm{b}, \mathrm{c}} \\
\left(\mathrm{K} \mathrm{km} \mathrm{s}^{-1}\right)\end{array}$ \\
\hline B & 061255.9 & 175742.4 & $6.25(0.01)$ & $0.45(0.01)$ & $2.51(0.16)$ & $6.83(0.22)$ & $0.94(0.16)$ & $0.97(0.09)$ & $<0.83$ & $<0.47$ & . & . \\
\hline 48 & 061311.0 & 175846.6 & $5.86(0.07)$ & $1.02(0.07)$ & $0.98(0.15)$ & $2.26(0.21)$ & $<0.76$ & $<0.42$ & $<0.81$ & $<0.45$ & . & . \\
\hline B & 061308.8 & 175821.6 & $6.62(0.03)$ & $0.42(0.03)$ & $0.95(0.16)$ & $2.34(0.22)$ & $<0.78$ & $<0.43$ & $<0.82$ & $<0.45$ & r. & 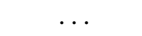 \\
\hline 49 & 061329.3 & 175533.2 & $7.85(0.03)$ & $0.54(0.03)$ & $0.94(0.15)$ & $2.56(0.21)$ & $0.70(0.15)$ & $0.50(0.08)$ & $<0.80$ & $<0.44$ & . & 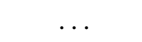 \\
\hline B & 061326.0 & 175608.5 & $8.03(0.01)$ & $0.29(0.02)$ & $1.50(0.15)$ & $2.41(0.21)$ & $<0.74$ & $<0.41$ & $<0.77$ & $<0.43$ & $\ldots$ & $\ldots$ \\
\hline $\mathrm{C}$ & 061332.2 & 175611.7 & $8.34(0.03)$ & $0.37(0.03)$ & $0.74(0.15)$ & $1.63(0.20)$ & $<0.76$ & $<0.42$ & $<0.75$ & $<0.42$ & $\cdots$ & $\cdots$ \\
\hline 50 & 061347.1 & 175455.3 & $9.18(0.01)$ & $0.44(0.01)$ & $3.43(0.16)$ & $11.20(0.22)$ & $1.02(0.16)$ & $1.08(0.09)$ & $<0.80$ & $<0.44$ & $\ldots$ & $\ldots$ \\
\hline B & 061345.4 & 175510.9 & $9.22(0.01)$ & $0.35(0.01)$ & $2.73(0.16)$ & $7.20(0.22)$ & $0.85(0.15)$ & $0.83(0.08)$ & $<0.76$ & $<0.42$ & $\ldots$ & $\ldots$ \\
\hline $\mathrm{C}$ & 061349.6 & 175429.4 & $9.63(0.01)$ & $0.36(0.01)$ & $2.63(0.15)$ & $7.28(0.20)$ & $0.75(0.14)$ & $0.50(0.08)$ & $<0.76$ & $<0.42$ & $\ldots$ & $\ldots$ \\
\hline 51 & 061359.3 & 175252.8 & $8.94(0.01)$ & $0.36(0.01)$ & $2.01(0.15)$ & $5.33(0.20)$ & $<0.73$ & $<0.41$ & $<0.73$ & $<0.41$ & .. & $\ldots$ \\
\hline 52 & 061409.7 & 174350.3 & $6.65(0.01)$ & $0.35(0.02)$ & $1.52(0.15)$ & $3.81(0.21)$ & $<0.76$ & $<0.42$ & $<0.79$ & $<0.44$ & $\ldots$ & $\ldots$ \\
\hline 54 & 061424.9 & 174503.9 & $7.90(0.02)$ & $0.53(0.02)$ & $1.69(0.15)$ & $5.80(0.20)$ & $0.88(0.15)$ & $0.83(0.08)$ & $<0.75$ & $<0.41$ & $\ldots$ & $\ldots$ \\
\hline B & 061424.1 & 174432.9 & $7.67(0.01)$ & $0.61(0.01)$ & $1.88(0.15)$ & $6.85(0.21)$ & $1.06(0.14)$ & $1.46(0.08)$ & $0.65(0.15)$ & $0.48(0.08)$ & $\ldots$ & .. \\
\hline $\mathrm{C}$ & 061423.7 & 174357.3 & $7.67(0.04)$ & $0.47(0.04)$ & $0.70(0.15)$ & $1.10(0.21)$ & $<0.75$ & $<0.41$ & $<0.78$ & $<0.43$ & $\cdots$ & $\cdots$ \\
\hline
\end{tabular}

Notes. Errors are given in parentheses.

${ }^{\mathrm{a}} \mathrm{B}$ and $\mathrm{C}$ denote multiple ammonia pointings that fall within a single $1.1 \mathrm{~mm}$ source.

${ }^{\mathrm{b}}$ Upper limits are $T_{\mathrm{mb}}<5 \mathrm{rms}$ and $W<5 \mathrm{rms} \Delta v / \sqrt{N}$, where $\Delta v$ is the width of a single channel in velocity, and $N$ is the number of pixels over which the average rms was calculated.

c “..." denotes sources that were not observed in the $\mathrm{NH}_{3}(4,4)$ transition. 
Table 6

Derived Gas Properties

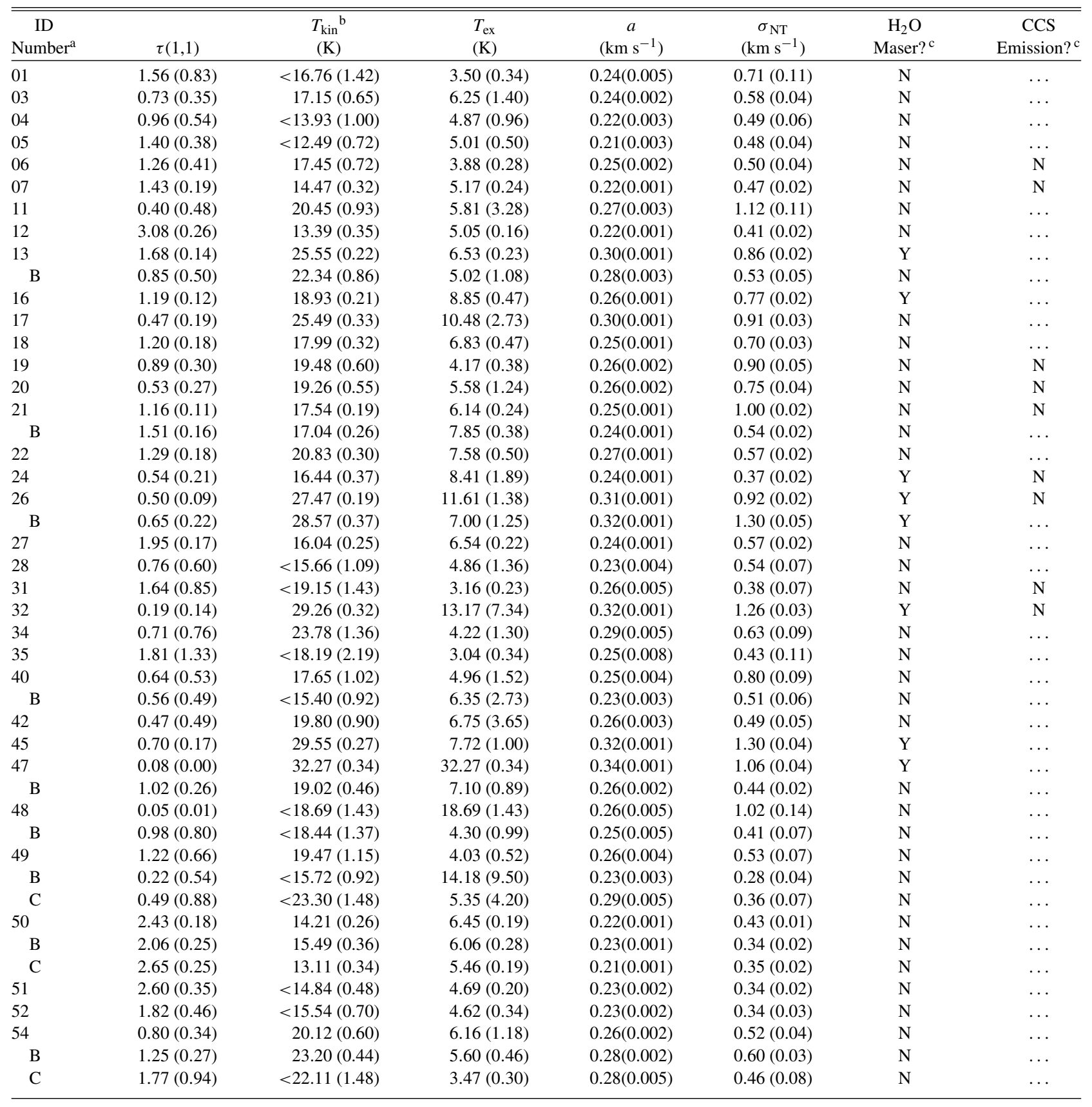

Notes. Errors are given in parentheses.

${ }^{\mathrm{a}} \mathrm{B}$ and $\mathrm{C}$ denote multiple ammonia pointings that fall within a single $1.1 \mathrm{~mm}$ source.

${ }^{\text {b }}$ Upper limits to $T_{\text {kin }}$ are due to a $<5 \sigma$ detection in the $\mathrm{NH}_{3}(2,2)$ transition.

c Either $\mathrm{CCS}$ or $\mathrm{H}_{2} \mathrm{O}$ was observed, but not both. "..." marks sources not observed in the given transition. Y denotes detected, $\mathrm{N}$ denotes not detected.

sources. The radial velocities observed in the $\mathrm{NH}_{3}$ spectra are listed in Table 5 (Column 4), and are shown in Figure 12. Filled circles mark sources in subset 1 , while the crosses mark sources without a $5 \sigma$ detection in the $\mathrm{NH}_{3}(2,2)$ line. The sources excluded from subset 1 are spread evenly in velocity space, as expected since the weaker BGPS sources should not be preferentially located at any given radial velocity. Toward the Galactic anti-center (as well as all Cardinal points: $l=0^{\circ}, 90^{\circ}, 180^{\circ}$, and $270^{\circ}$ ), radial velocities expected from purely circular rotation are $0 \mathrm{~km} \mathrm{~s}^{-1}$, rendering kinematic distances unsound. A non-zero radial velocity along these lines of sight is most likely due to the peculiar motions of individual gas clouds rather than galactic rotation.

From Figure 12, it is apparent that groups 2 and 3 span the same velocity range $\left(5-10 \mathrm{~km} \mathrm{~s}^{-1}\right)$ while group 1 is found at slightly lower radial velocities ( -1 to $4 \mathrm{~km} \mathrm{~s}^{-1}$ ). Although there is no evidence in our data that these groups are related, surveys of lower density tracers (such as CO and ${ }^{13} \mathrm{CO}$ ) toward S247 and S252 (groups 1 and 2) show evidence of interaction between the two H II regions. Kömpe et al. (1989) mapped the S247/252 complex in ${ }^{13} \mathrm{CO}(J=1-0)$ transition with a beam-sampled map at $4^{\prime}$ resolution and found a bridge connecting the two 

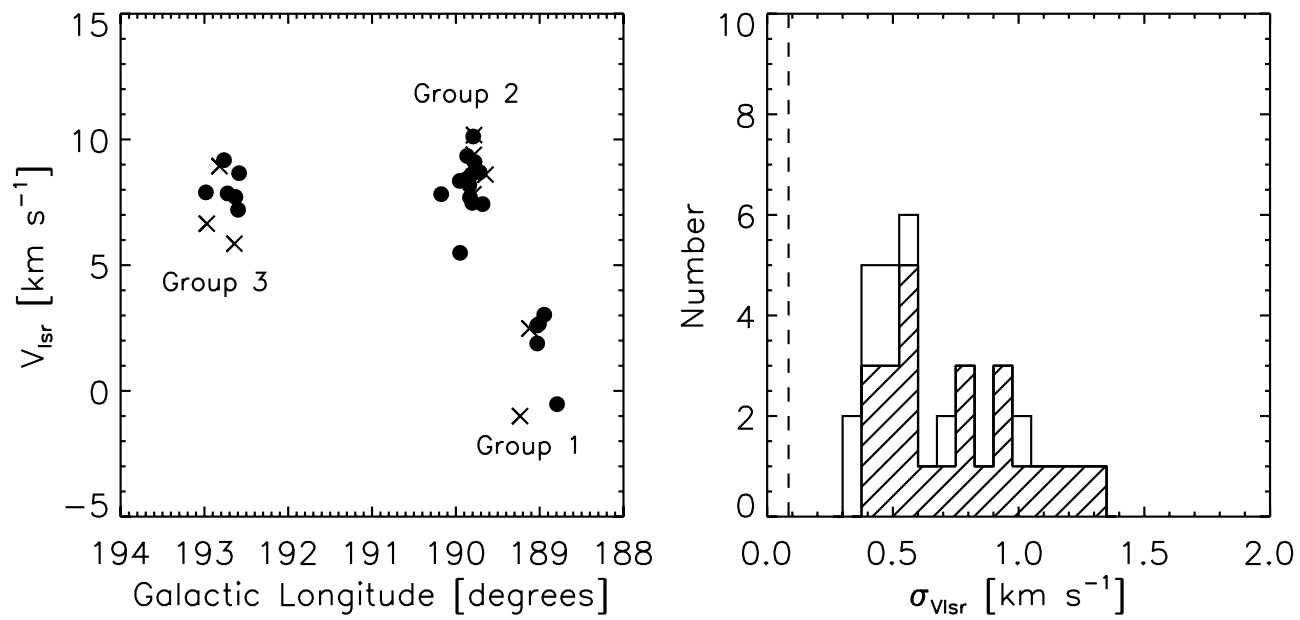

Figure 12. Left: velocity with respect to the LSR vs. Galactic longitude. Groups 2 and 3 span the same velocity range, while group 1 is found at lower velocities. Right: distribution of velocity dispersions. The white histogram marks the full sample and the dashed histogram marks subset 1 . The dashed line marks the spectral resolution of the $\mathrm{NH}_{3}$ spectra, $0.08 \mathrm{~km} \mathrm{~s}^{-1}$.

clouds. Specifically, the bulk of the material toward S247 is found between velocities of $-1 \mathrm{~km} \mathrm{~s}^{-1}$ and $5 \mathrm{~km} \mathrm{~s}^{-1}$ with a tail extending to velocities of up to $10 \mathrm{~km} \mathrm{~s}^{-1}$, which overlaps the $\mathbf{S} 252$ region in velocity space (see Figure 7 in Kömpe et al. 1989). Carpenter et al. (1995a) mapped a larger region including the S247 and S252 complexes in ${ }^{13} \mathrm{CO}(J=1-0)$ and found three distinct filaments. Two filaments with radial velocities of $\sim 2 \mathrm{~km} \mathrm{~s}^{-1}$ wrap around S247, while the third filament, at a radial velocity of $\sim 10 \mathrm{~km} \mathrm{~s}^{-1}$, extends to the S252 region. It is clear that the S247 and S252 regions (groups 1 and 2) are interacting, justifying our application of the VLBI parallax distance to our entire sample regardless of the difference in radial velocities between the groups of sources.

Not all sources will be at precisely the parallax distance; however, we account for variation in the distances by including a larger error than the parallax distance error. Since it is clear that groups 1 and 2 are part of filaments wrapping around and interacting between S247 and S252, we will consider the size of the H II regions when determining the spread in distance. S247 has a diameter of $9^{\prime}$ and S252 has a diameter of 40' (Sharpless 1959). If the cloud between groups 1 and 2 is assumed to be spherically symmetric, its angular size on the sky can provide an estimate of the distance along the line of sight. Since it is apparent that groups 1 and 2 are interacting, we will consider a sphere of approximately $100^{\prime}$ in diameter that encompasses both groups 1 and 2 and the associated $\mathrm{H}$ II regions, and corresponds to a distance uncertainty of $0.061 \mathrm{kpc}$. We will assume the spread in distance is approximately equal to the $100^{\prime}$ diameter, and will assign that to the distance error to account for the case where the parallax source is found at the extreme edge of the region and note that this is likely a minimum estimate of the error.

\subsection{Kinetic Temperature}

The kinetic temperatures derived from the $\mathrm{NH}_{3}$ observations are given in Column 3 of Table 6. The uncertainties in individual determinations of $T_{K}$ are small, with $\left\langle\sigma_{T_{K}} / T_{K}\right\rangle=0.04$. In this and subsequent analyses, the uncertainties quoted are the standard deviation about the mean of the sample, rather than the uncertainty in the mean. For sources without a $5 \sigma$ detection in $\mathrm{NH}_{3}(2,2)$, the derived kinetic temperature is an upper limit, and thus the mean kinetic temperature for the full sample is only an upper limit. The distribution for the full sample is characterized by $\left\langle T_{K}\right\rangle<19.4 \pm 4.9 \mathrm{~K}$, while the distribution for subset 1 is characterized by $\left\langle T_{K}\right\rangle=20.6 \pm 5.1 \mathrm{~K}$. The mean limit on kinetic temperature for the sources without a $5 \sigma \mathrm{NH}_{3}(2,2)$ detection is $\left\langle T_{K}\right\rangle<16.1 \pm 2.3 \mathrm{~K}$. As some property distributions may be non-Gaussian, the uncertainty should be regarded primarily as an indicator of the scatter in the sample. The dispersion about the mean kinetic temperature for the full sample is thus six times larger $(0.24)$ than the fractional uncertainties in individual temperatures, so the differences among the sources are real.

Zinchenko et al. (1997) mapped 17 molecular clouds associated with FIR sources in $\mathrm{NH}_{3}(1,1)$ and $(2,2)$ and found that $T_{K}$ increases toward the peak of the $\mathrm{NH}_{3}$ emission. Additionally, Friesen et al. (2009) have shown that $\mathrm{NH}_{3}$ emission closely follows millimeter continuum emission. Since our $\mathrm{NH}_{3}$ pointings are located near the peak of the millimeter emission, which corresponds to the $\mathrm{NH}_{3}$ peak as well, the mean $T_{K}$ is likely smaller than the peak $T_{K}$ we report.

\subsection{Velocity Dispersion and Non-thermal Pressures}

The line width provides a measure of the one-dimensional velocity dispersion within each source. Rosolowsky et al. (2008) found velocity dispersions ranging from 0.07 to $0.7 \mathrm{~km} \mathrm{~s}^{-1}$ for the $\mathrm{NH}_{3}(1,1)$ and $(2,2)$ lines of low-mass star-forming cores in the Perseus molecular cloud, and they suggest that there is evidence of multiple velocity components in all sources with $\sigma_{v}>0.2 \mathrm{~km} \mathrm{~s}^{-1}$. The velocity dispersions are listed in Column 5 of Table 5 and the distribution of our sample is shown in Figure 12. In contrast to the low-mass sources in Perseus, our distribution has a tail extending up to a high velocity dispersion $\sigma_{v} \approx 1.3 \mathrm{~km} \mathrm{~s}^{-1}$.

The observed velocity dispersion is a combination of the thermal and non-thermal motions of the gas. In order to calculate the non-thermal contribution, $\sigma_{\mathrm{NT}}$, we simply remove the thermal contribution:

$$
\sigma_{\mathrm{NT}}=\sqrt{\sigma_{v}^{2}-\frac{k T_{K}}{17 m_{H}}},
$$

where $\left(k T_{K} / 17 m_{H}\right)^{1 / 2}$ is the thermal broadening due to $\mathrm{NH}_{3}$ and $17 m_{H}$ is the mass of a single ammonia molecule. The non-thermal velocity dispersion ranges from $0.37 \mathrm{~km} \mathrm{~s}^{-1}$ to $1.3 \mathrm{~km} \mathrm{~s}^{-1}$ with a mean of $0.72 \pm 0.25 \mathrm{~km} \mathrm{~s}^{-1}$ for subset 1 . We can compare $\sigma_{\mathrm{NT}}$ to the predicted thermal sound speed of the gas given by $a=\left(k T_{K} / \mu m_{H}\right)^{1 / 2}$, where $\mu=2.37$. The 


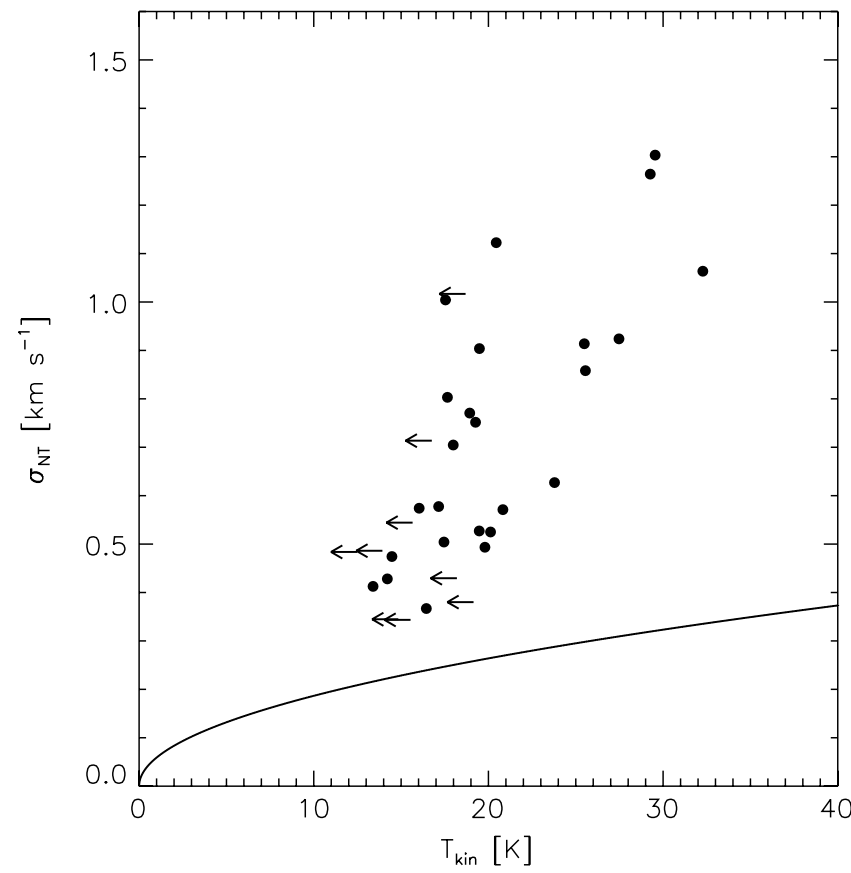

Figure 13. Non-thermal velocity dispersion, $\sigma_{\mathrm{NT}}$, vs. derived kinetic temperature. Arrows indicate upper limits for the kinetic temperatures. Solid line denotes the thermal sound speed given by $a=\sqrt{k T / \mu m_{H}}$.

thermal sound speed for subset 1 ranges from $0.22 \mathrm{~km} \mathrm{~s}^{-1}$ to $0.34 \mathrm{~km} \mathrm{~s}^{-1}$ with a mean of $0.27 \pm 0.03 \mathrm{~km} \mathrm{~s}^{-1}$. The mean Mach number is then 2.8. Both $a$ and $\sigma_{\mathrm{NT}}$ are listed in Table 6 (Columns 5 and 6). The corresponding ratio of $\sigma_{\mathrm{NT}}$ to $a$ ranges from 1.5 to 4.2 with a mean of $2.7 \pm 0.8$. The non-thermal velocity dispersion is shown in Figure 13 versus $T_{K}$. The solid line plots the thermal sound speed, $a$, as a function of $T_{K}$. In all sources, the non-thermal velocity dispersion is much larger than the thermal velocity dispersion and the discrepancy increases with $T_{K}$

There is no apparent correlation between $\sigma_{\mathrm{NT}}$ and source size or mass. However, there is a correlation between the position of a source within its group and $\sigma_{\mathrm{NT}}$. Figure 14 displays the position of each source in Galactic coordinates and the symbol size denotes the magnitude of $\sigma_{\mathrm{NT}}$. For group 2, the highest values of $\sigma_{\mathrm{NT}}$ are found at the center of the cluster. A similar statement could be made for group 3, but there it is difficult to definitively say, due to the smaller number of sources.

\subsection{Virial and Isothermal Masses}

Combining heterodyne and dust continuum observations provides estimates of both virial mass and total gas and dust mass. Total gas and dust mass (also called the isothermal mass, $M_{\text {iso }}$, due to the simplifying assumption that the dust can be characterized by a single temperature) is given by

$$
\begin{aligned}
M_{\mathrm{iso}} & =\frac{S_{v} D^{2}}{\kappa_{\nu} B_{v}\left(T_{d}\right)} \\
& =13.1 M_{\odot}\left(\frac{S_{v}}{1 \mathrm{Jy}}\right)\left(\frac{D}{1 \mathrm{kpc}}\right)^{2}\left(\frac{e^{13.0 \mathrm{~K} / T_{d}}-1}{e^{13.0 \mathrm{~K} / 20.0 \mathrm{~K}}-1}\right),
\end{aligned}
$$

where $S_{v}$ is flux density, $D$ is the distance, $\kappa_{v}$ is the dust opacity per gram of gas and dust, and $B_{v}$ is the Planck function evaluated at a dust temperature, $T_{d}$. We logarithmically interpolate the Ossenkopf \& Henning (1994) dust opacities (Table 1, Column 5 , commonly referred to as $\mathrm{OH} 5$ dust) to the effective central

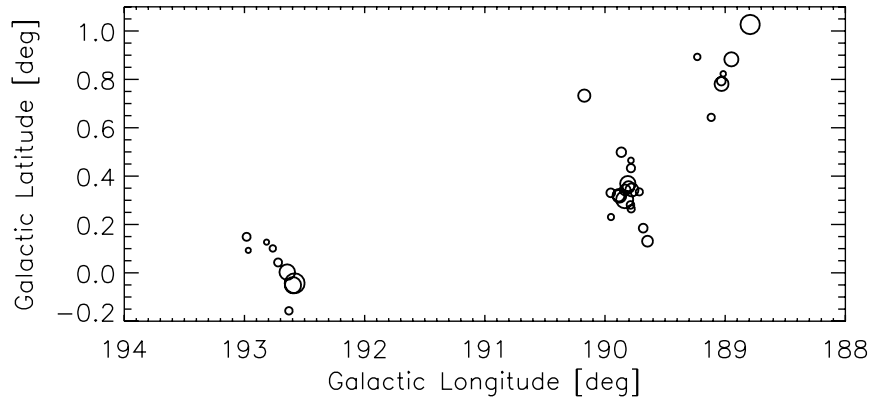

Figure 14. Galactic longitude vs. Galactic latitude for the full sample. Symbol size denotes the magnitude of the non-thermal velocity dispersion, $\sigma_{\mathrm{NT}}$.

frequency of the Bolocam bandpass convolved with a $20 \mathrm{~K}$ blackbody modified by an opacity varying with frequency as $\kappa_{v} \propto v^{1.8}(271.1 \mathrm{GHz}$; Aguirre et al. 2010) and obtain $\kappa_{v}=0.0114 \mathrm{~cm}^{2} \mathrm{~g}^{-1}$.

We assume the gas and dust are collisionally coupled, make the simplifying assumption that $T_{d}=T_{K}$ and use $T_{K}$ derived from the $\mathrm{NH}_{3}$ observations. Evans et al. (1977) observed the $\mathrm{S} 255$ molecular cloud in ${ }^{12} \mathrm{CO},{ }^{13} \mathrm{CO}, \mathrm{H}_{2} \mathrm{CO}$, and $2.2 \mu \mathrm{m}$ emission. They studied the gas and dust energetics and concluded that the primary energy flow is through infrared dust emission and that an embedded source and the exciting stars of the many nearby $\mathrm{H}$ II regions are the likely heat sources for the cloud. Dust typically controls the energetics in the dense interior of the star-forming regions where the density is high enough to shield the gas from the ultraviolet radiation in the interstellar radiation field (ISRF) and also to transfer energy from the dust to the gas via collisions (Goldreich \& Kwan 1974; Evans et al. 2001). Toward the outer edges of the clump, the density is much lower, and the gas is no longer collisionally coupled to the dust. The gas is also now subject to the ultraviolet portion of the ISRF, allowing the gas kinetic temperature to rise above the dust temperature (see Figure 9 in Mueller et al. 2002). The BGPS observations probe all of these regions, and detailed models will be needed to include temperature gradients. For now, we rely on previous modeling (e.g., Mueller et al. 2002; Shirley et al. 2002) to support the assumption that $T_{d}=T_{K}$ on average.

As discussed in Section 6.2, our single measurement of $T_{K}$ likely corresponds to the maximum temperature seen within the BGPS source. Thus, using $T_{d}=T_{K}$ to describe the dust temperature for the entire source will result in a lower limit for the isothermal mass. Zinchenko et al. (1997) find a maximum $T_{K}$ of $28 \mathrm{~K}$ at the peak of the $\mathrm{NH}_{3}$ emission, and a minimum $T_{K}$ of $15 \mathrm{~K}$ at the edge of the $\mathrm{NH}_{3}$ emission. This difference in temperatures corresponds to a factor of 2.3 increase in mass if the minimum temperature is assumed to be $T_{d}$. If we assume mean values from Zinchenko et al. (1997) of $T_{K}=24$ and $17.5 \mathrm{~K}$, the mass would increase by a factor of 1.5 assuming the minimum temperature as $T_{d}$ rather than the maximum. If we are observing the maximum $T_{K}$ then our calculated values of $M_{\text {iso }}$ are likely to underestimate the mass by a factor of up to approximately 2 .

The virial mass for a spherical, uniform density gas cloud is given by

$$
M_{\mathrm{vir}}=\frac{5 \sigma_{v}^{2} R}{G},
$$

where $\sigma_{v}$ is the Gaussian 1/e width of the line (model fit for the velocity dispersion), $R$ is the source radius, and $G$ is the gravitational constant. We assume that the velocity dispersion measured with our single $\mathrm{NH}_{3}$ pointing is also applicable at the edge of the $1.1 \mathrm{~mm}$ continuum emission, and use the 
Table 7

Derived Masses and Densities

\begin{tabular}{|c|c|c|c|c|c|c|c|c|c|}
\hline $\begin{array}{c}\text { ID } \\
\text { Number }\end{array}$ & $\begin{array}{c}M_{\text {iso }}\left(120^{\prime \prime}\right) \\
\left(M_{\odot}\right)\end{array}$ & $\begin{array}{c}M_{\text {iso }} \text { (int) } \\
\left(M_{\odot}\right)\end{array}$ & $\begin{array}{c}M_{\mathrm{Vir}}(\mathrm{int}) \\
\left(M_{\odot}\right)\end{array}$ & $\begin{array}{c}n_{p}(\text { int }) \\
\left(10^{3} \mathrm{~cm}^{-3}\right)\end{array}$ & $\begin{array}{c}n_{\mathrm{ex}} \\
\left(10^{3} \mathrm{~cm}^{-3}\right)\end{array}$ & $\begin{array}{c}\Sigma(\text { int }) \\
\left(10^{-2} \mathrm{~g} \mathrm{~cm}^{-2}\right)\end{array}$ & $\begin{array}{c}N_{\mathrm{NH}_{3}} \\
\left(10^{14} \mathrm{~cm}^{-2}\right)\end{array}$ & $\begin{array}{c}N_{\mathrm{H}_{2}} \text { (int) } \\
\left(10^{21} \mathrm{~cm}^{-2}\right)\end{array}$ & $\begin{array}{c}X_{\mathrm{NH}_{3}} \\
\left(10^{-8}\right) \\
\end{array}$ \\
\hline 01 & $69(7)$ & $73(6)$ & $150(28)$ & $2.39(0.33)$ & $1.32(0.25)$ & $1.95(0.08)$ & $0.53(0.14)$ & $4.17(0.48)$ & $1.26(0.37)$ \\
\hline 03 & $108(14)$ & $121(13)$ & $96(10)$ & $4.31(0.64)$ & $8.29(0.99)$ & $3.42(0.18)$ & $2.31(0.31)$ & $7.32(0.94)$ & $3.16(0.59)$ \\
\hline 04 & 134(12) & 115(9) & $57(8)$ & $7.18(0.96)$ & $4.82(0.79)$ & $4.73(0.15)$ & $0.78(0.09)$ & 10.11(1.10) & $0.77(0.12)$ \\
\hline 05 & 137(13) & $126(19)$ & $67(7)$ & $4.52(0.63)$ & $5.35(0.50)$ & $3.58(0.21)$ & $4.01(0.63)$ & $7.65(0.89)$ & $5.24(0.35)$ \\
\hline 06 & $113(15)$ & 71(11) & $49(5)$ & $8.54(1.54)$ & $2.07(0.22)$ & $4.52(0.46)$ & $2.28(0.41)$ & $9.66(1.58)$ & $2.36(0.57)$ \\
\hline 07 & $124(21)$ & $106(18)$ & $60(4)$ & $4.86(0.97)$ & $5.29(0.24)$ & $3.55(0.42)$ & $3.55(0.32)$ & $7.59(1.40)$ & $4.68(0.96)$ \\
\hline 11 & 139(13) & $99(12)$ & $436(56)$ & $1.98(0.32)$ & $6.65(1.28)$ & $1.91(0.13)$ & $0.95(0.03)$ & $4.08(0.57)$ & $2.34(0.34)$ \\
\hline 12 & $172(23)$ & $197(24)$ & $59(4)$ & $4.23(0.68)$ & $4.35(0.14)$ & $3.97(0.26)$ & $7.04(0.38)$ & $8.49(1.20)$ & $8.29(1.25)$ \\
\hline 13 & $373(40)$ & $465(39)$ & $304(18)$ & $5.58(0.74)$ & $6.80(0.21)$ & $6.36(0.12)$ & $7.58(0.33)$ & $13.59(1.47)$ & $5.57(0.65)$ \\
\hline 16 & $502(53)$ & $292(28)$ & $153(8)$ & $14.29(2.02)$ & $16.32(0.53)$ & $10.19(0.35)$ & $6.65(0.37)$ & $21.79(2.58)$ & $3.05(0.40)$ \\
\hline 17 & $463(30)$ & $468(39)$ & $244(17)$ & $15.69(2.46)$ & $20.09(1.46)$ & $12.69(0.24)$ & $3.53(1.08)$ & $27.13(2.01)$ & $1.30(0.27)$ \\
\hline 18 & $386(38)$ & $429(37)$ & $208(15)$ & $4.94(0.66)$ & $9.38(0.46)$ & $5.70(0.12)$ & $4.93(0.45)$ & $12.19(1.33)$ & $4.04(0.58)$ \\
\hline 19 & $143(15)$ & $143(15)$ & 291(27) & $2.64(0.39)$ & $2.68(0.25)$ & $2.61(0.12)$ & $2.92(0.46)$ & $5.58(0.70)$ & $5.24(1.05)$ \\
\hline 20 & $149(16)$ & $126(14)$ & $187(17)$ & $2.95(0.45)$ & $6.10(0.62)$ & $2.70(0.14)$ & $0.78(0.44)$ & $5.76(0.77)$ & $1.35(0.78)$ \\
\hline 21 & $560(57)$ & $779(66)$ & $560(31)$ & $3.76(0.50)$ & $7.46(0.22)$ & $5.80(0.12)$ & $6.22(0.35)$ & $12.41(1.35)$ & $5.01(0.61)$ \\
\hline 22 & $608(61)$ & $365(35)$ & $109(7)$ & $8.56(1.21)$ & $10.67(0.49)$ & $7.80(0.26)$ & $4.54(0.40)$ & $16.68(1.97)$ & $2.72(0.40)$ \\
\hline 24 & 204(19) & $125(18)$ & $32(2)$ & $8.48(1.13)$ & $17.81(1.38)$ & $5.43(0.11)$ & $1.51(0.37)$ & $11.62(1.13)$ & $1.30(0.13)$ \\
\hline 26 & $492(31)$ & $556(20)$ & $277(8)$ & $13.69(0.72)$ & $23.37(0.79)$ & $12.26(0.19)$ & $4.21(0.52)$ & $26.22(1.17)$ & $1.60(0.21)$ \\
\hline 27 & $471(47)$ & $853(65)$ & 194(13) & $3.58(0.46)$ & $8.44(0.26)$ & $5.79(0.06)$ & $6.72(0.31)$ & $12.38(1.27)$ & $5.43(0.61)$ \\
\hline 28 & $117(10)$ & $150(12)$ & $116(19)$ & $2.17(0.28)$ & $4.63(0.92)$ & $2.32(0.05)$ & $0.65(0.06)$ & $4.97(0.51)$ & $1.30(0.18)$ \\
\hline 31 & $56(7)$ & $58(7)$ & $42(8)$ & $2.29(0.37)$ & $0.69(0.13)$ & $1.75(0.13)$ & $0.16(0.06)$ & $3.75(0.54)$ & $0.41(0.18)$ \\
\hline 32 & $149(15)$ & $164(16)$ & $459(28)$ & $5.72(0.99)$ & $29.86(1.92)$ & $4.56(0.31)$ & $1.82(0.03)$ & $9.76(0.75)$ & $1.86(0.15)$ \\
\hline 34 & $176(9)$ & $319(10)$ & $231(42)$ & $1.37(0.15)$ & $2.69(0.70)$ & $2.20(0.07)$ & $0.42(0.02)$ & $4.70(0.36)$ & $0.89(0.08)$ \\
\hline 35 & $30(7)$ & $28(4)$ & $22(6)$ & $14.41(2.66)$ & $0.49(0.13)$ & $4.69(0.66)$ & $0.14(0.02)$ & $10.04(1.68)$ & $0.14(0.03)$ \\
\hline 40 & $105(10)$ & 143(9) & $186(26)$ & $4.98(0.61)$ & $4.67(0.88)$ & $3.98(0.06)$ & $0.80(0.05)$ & $8.50(0.82)$ & $0.94(0.11)$ \\
\hline 42 & $46(11)$ & $32(8)$ & $44(6)$ & $4.81(1.32)$ & $9.35(1.77)$ & $2.37(0.52)$ & $0.65(0.03)$ & $5.08(1.34)$ & $1.28(0.34)$ \\
\hline 45 & $509(53)$ & $397(32)$ & $456(28)$ & $16.96(2.21)$ & $10.00(0.57)$ & $12.65(0.22)$ & $5.74(0.81)$ & $27.05(2.86)$ & $2.12(0.38)$ \\
\hline 47 & 481(49) & $540(43)$ & $552(37)$ & $3.90(0.51)$ & $\ldots$ & $5.26(0.08)$ & $2.23(0.03)$ & $11.25(1.18)$ & $1.98(0.21)$ \\
\hline 48 & $84(9)$ & $87(8)$ & $310(53)$ & $2.67(0.38)$ & $\ldots$ & $2.23(0.09)$ & $0.68(0.05)$ & $4.76(0.57)$ & $1.43(0.20)$ \\
\hline 49 & $138(11)$ & 214(13) & $126(21)$ & $2.04(0.24)$ & $2.30(0.41)$ & $2.51(0.00)$ & $0.51(0.14)$ & $5.36(0.50)$ & $0.95(0.28)$ \\
\hline 50 & 191(28) & $233(32)$ & $64(4)$ & $4.74(0.80)$ & $8.54(0.24)$ & $4.53(0.35)$ & $6.78(0.28)$ & $9.69(1.47)$ & $7.00(1.10)$ \\
\hline 51 & $54(20)$ & $44(13)$ & $29(2)$ & $2.92(0.90)$ & $3.52(0.18)$ & $1.89(0.03)$ & $4.29(0.28)$ & $4.04(1.20)$ & $10.60(3.22)$ \\
\hline 52 & $56(17)$ & $51(13)$ & $23(3)$ & $6.47(1.81)$ & $3.56(0.33)$ & $3.36(0.75)$ & $2.85(0.34)$ & $7.19(1.93)$ & $3.96(1.16)$ \\
\hline 54 & $245(23)$ & $371(27)$ & $128(13)$ & $3.25(0.41)$ & $7.29(0.81)$ & $4.11(0.05)$ & $2.17(0.65)$ & $8.78(0.88)$ & $2.47(0.78)$ \\
\hline
\end{tabular}

Note. Errors are given in parentheses.

single measured value in conjunction with the millimeterderived object radius, $R_{\mathrm{obj}}$, to calculate the virial mass. This assumption is supported by Friesen et al. (2009) who found that $\mathrm{NH}_{3}$ emission closely traces $850 \mu \mathrm{m}$ continuum emission and covers the same spatial extent as the cold dust producing the continuum emission. However, Zinchenko et al. (1997) found that the ammonia line width increased toward the peak of the $\mathrm{NH}_{3}$ emission for about half of their sample. Thus, we are likely overestimating the virial mass by using the line width measured near the peak.

$M_{\text {vir }}, M_{\text {iso }}\left(120^{\prime \prime}\right)$, and $M_{\text {iso }}$ (int) are given in Columns 2-4 of Table 7. For subset 1 , the isothermal masses within a $120^{\prime \prime}$ aperture range from $47 M_{\odot}$ to $610 M_{\odot}$ with a mean of $280 \pm$ $180 M_{\odot}$. Similarly, $M_{\text {iso }}$ (int) ranges from $33 M_{\odot}$ to $850 M_{\odot}$ with a mean of $300 \pm 220 M_{\odot}$. The virial masses range from $33 M_{\odot}$ to $560 M_{\odot}$ with a mean of $220 \pm 160 M_{\odot}$. The distributions of $M_{\text {iso }}\left(120^{\prime \prime}\right)$ and $M_{\text {iso }}$ (int) are shown in Figure 15. The mean $M_{\text {vir }}$ to $M_{\text {iso }}$ (int) ratio is $0.96 \pm 0.93$ for subset 1 . The ratio of $M_{\text {vir }}$ to $M_{\text {iso }}$ (int) is shown versus $M_{\text {iso }}$ (int) in Figure 16 . The dashed line marks the mean ratio of 1.0. The trend of decreasing $M_{\text {vir }} / M_{\text {iso }}$ reflects the dependence of the ratio on the inverse of the source size at a given distance. $R_{\text {obj }}$ increases with $M_{\text {iso }}$ (int) which lowers $M_{\text {vir }} / M_{\text {iso }}$ as a function of $M_{\text {iso }}$ (int).

In these calculations, we have made the simplifying assumption that the density is uniform. However, if the emission is not spherical or if the density follows a power law the virial mass will differ. A corrected virial mass is given by

$$
\begin{gathered}
M_{\mathrm{vir}}=\frac{5 \sigma_{v}^{2} R}{a_{1} a_{2} G}, \\
a_{1}=\frac{1-p / 3}{1-2 p / 5} \text { for } p<2.5
\end{gathered}
$$

(Bertoldi \& McKee 1992), where $a_{1}$ is the correction for a power-law density distribution, $a_{2}$ is the correction for a nonspherical shape, and $p$ is the density power-law index from $n(r)=n_{f}\left(r / r_{f}\right)^{-p}$ (Mueller et al. 2002). The mass ratio is then given by

$$
\frac{M_{\mathrm{vir}}}{M_{\mathrm{iso}}}=\frac{\sigma_{v}^{2} \theta_{\mathrm{rad}} B_{v}\left(T_{d}\right) \kappa_{v}}{2 G S_{v} D a_{1} a_{2}} \propto \frac{\kappa_{v}}{D a_{1} a_{2}} .
$$

The mean aspect ratio of our sources is $\left\langle R_{\mathrm{maj}} / R_{\min }\right\rangle=1.5 \pm 0.3$, which gives $a_{2}=1.1$. To estimate the effect of a power-law density distribution, we adopt the mean power-law index found by Mueller et al. (2002), $\langle p\rangle=1.8 \pm 0.4$, which was derived by modeling the emission from 31 massive star-forming regions, and find $a_{1}=1.43 \pm 0.34$. If the corrections for a powerlaw density distribution (Equation (8)) and for a non-spherical shape are included, then $\left\langle M_{\mathrm{vir}} / M_{\mathrm{iso}}\right\rangle$ would be decreased by 


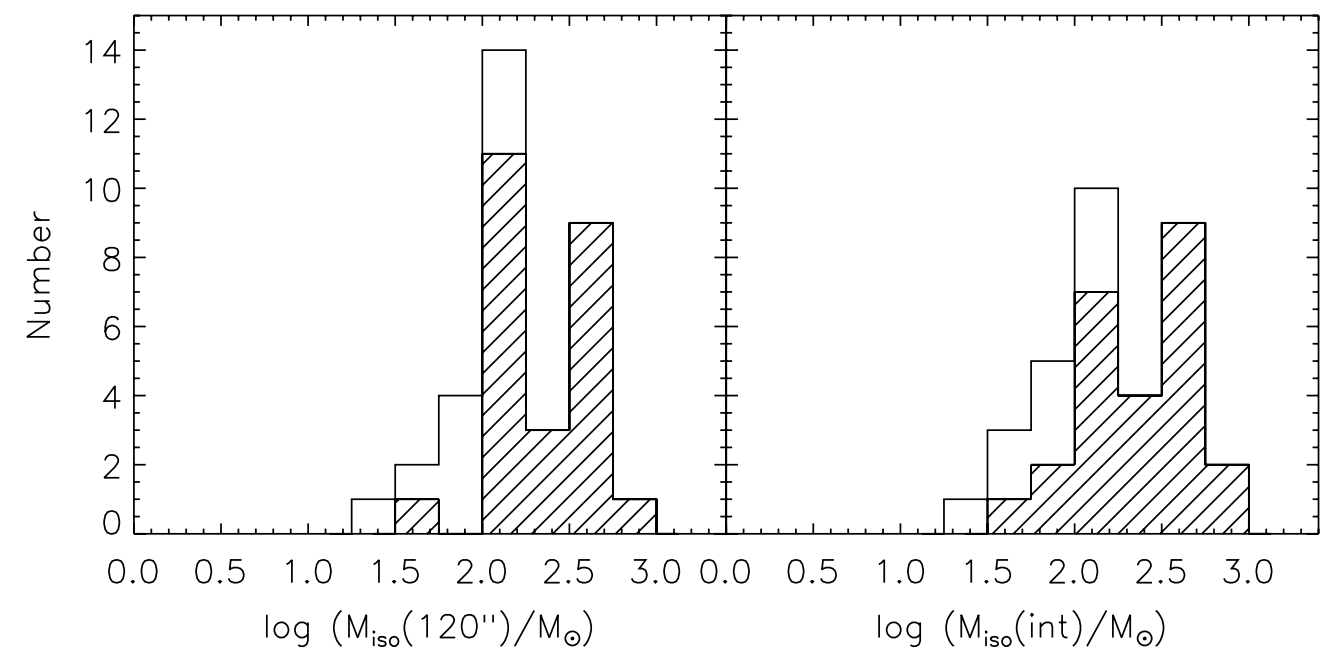

Figure 15. Distributions of $M_{\text {iso }}$ derived from $S_{v}\left(120^{\prime \prime}\right.$ ) (left) and $S_{v}$ (int) (right). The white histogram marks the full sample and the striped histogram marks subset 1 .

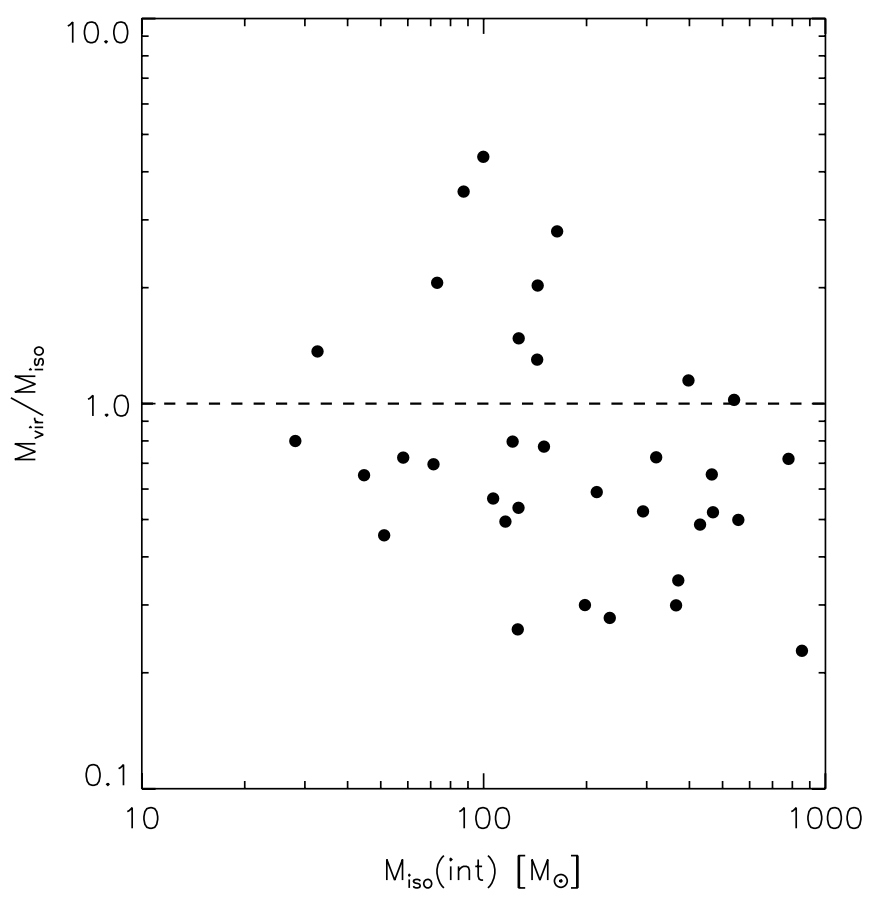

Figure 16. Virial parameter $\left(M_{\mathrm{vir}} / M_{\mathrm{iso}}\right)$ vs. $M_{\text {iso }}($ int $)$. The dashed line marks the mean virial parameter of 1 .

$36 \%$ to $0.61 \pm 0.59$ for subset 1 . However, since we do not have accurate values of the power-law index for our individual sources, we will not include the correction in our calculations or subsequent discussion. Magnetic fields are also excluded from our virial mass calculation, but if magnetic fields provide additional support to the cloud, then the true mass would be larger than given by Equation (6) or (7).

The observed scatter in $M_{\text {vir }}$ to $M_{\text {iso }}$ (Figure 16) could be a result of deviations from the assumed distance of $2.10 \mathrm{kpc}$ or deviations from Equation (6) due to different power-law density distributions or source ellipticities, all of which could differ from source to source and produce the observed scatter. Additional assumptions which could produce the observed scatter include the assumptions that the sources are virialized and that the velocity dispersion measured at the peak of the millimeter emission is also valid at $R_{\mathrm{obj}}$. A virial parameter, $M_{\mathrm{vir}} / M_{\mathrm{iso}}$, of 1 suggests that both mass estimates are reasonable.

\subsection{Volume and Surface Densities}

Average particle density and average surface density can be calculated based on a mean particle weight per $\mathrm{H}$ nucleus $\left(\mu_{p}=2.37\right)$ and a total mass within a given physical size, assuming a uniform density. We assumed a spherical volume given by $4 \pi R_{\mathrm{obj}}^{3} / 3$. Mean number densities of particles and mass surface densities were calculated for each source (see Column 5 of Table 7). For subset $1, n_{p}$ (int) ranges from $1.4 \times 10^{3} \mathrm{~cm}^{-3}$ to $17.0 \times 10^{3} \mathrm{~cm}^{-3}$ with a mean of $6.2 \pm 4.4 \times 10^{3} \mathrm{~cm}^{-3}$, and the characteristic values for the full sample are given in Table 8 .

The distribution of derived surface densities, $\Sigma$ (int), ranges from $0.019 \mathrm{~g} \mathrm{~cm}^{-2}$ to $0.13 \mathrm{~g} \mathrm{~cm}^{-2}$ with a mean of $0.055 \pm$ $0.032 \mathrm{~g} \mathrm{~cm}^{-2}$, for subset 1 . The mean surface density for the full sample is $0.048 \pm 0.030 \mathrm{~g} \mathrm{~cm}^{-2}$ or $230 \pm 140 M_{\odot} \mathrm{pc}^{-2}$ which is similar to the gas surface density threshold for star formation of $200 M_{\odot} \mathrm{pc}^{-2}$ seen in nearby, low-mass star-forming clouds (A. Heiderman et al. 2010, in preparation). This similarity supports the idea that our BGPS sources are sites of star formation, while the low volume densities imply we are detecting the less dense, larger scale portions of clumps rather than the higher density cores.

There is also evidence that a few of the BGPS clumps in this region include multiple higher density sources when observed with interferometers (see Section 7). Eight of the 34 BGPS cores also have water maser emission (see Table 6) which requires densities greater than $10^{10} \mathrm{~cm}^{-3}$ (Strelnitskii 1984; Elitzur et al. 1989), suggesting that the clumps contain much higher density substructure.

\subsection{Excitation Temperature}

The measured excitation temperatures are plotted against the kinetic temperatures in Figure 17. The filled circles represent subset 1 , and the corresponding distribution is characterized by $\left\langle T_{\mathrm{ex}}(1,1)\right\rangle=7.8 \pm 5.6 \mathrm{~K}$. There are two sources where the bestfitting model requires the limiting case $T_{\mathrm{ex}}=T_{K}$, and higher quality data would be needed to constrain $T_{\mathrm{ex}}$.

The mean difference between kinetic and excitation temperatures for subset 1 is $\left\langle T_{K}-T_{\text {ex }}\right\rangle=12.8 \pm 4.5 \mathrm{~K}$. There are two possible explanations for the differences in derived temperatures. The first possibility is inherent in the way we measure the excitation temperature. As described in Section 4.2, in order to calculate $T_{\mathrm{ex}}$ we must assume something about the ratio of the solid angle of the source to that of the beam, which here we 
Table 8

Statistical Summary

\begin{tabular}{|c|c|c|c|c|c|c|c|c|c|}
\hline \multirow[b]{2}{*}{ Property } & \multirow[b]{2}{*}{ Units } & \multicolumn{4}{|c|}{ Full Sample } & \multicolumn{4}{|c|}{ Subset 1} \\
\hline & & Minimum & Mean $^{\mathrm{a}}$ & Median & Maximum & Minimum & Mean $^{\mathrm{a}}$ & Median & Maximum \\
\hline$R_{\text {maj }}$ & $\mathrm{pc}$ & 0.18 & $0.35(0.10)$ & 0.34 & 0.55 & 0.22 & $0.37(0.10)$ & 0.35 & 0.55 \\
\hline$R_{\min }$ & $\mathrm{pc}$ & 0.13 & $0.23(0.05)$ & 0.23 & 0.37 & 0.16 & $0.24(0.05)$ & 0.23 & 0.37 \\
\hline$R_{\mathrm{obj}}$ & $\mathrm{pc}$ & 0.20 & $0.56(0.19)$ & 0.51 & 0.99 & 0.30 & $0.60(0.19)$ & 0.56 & 0.99 \\
\hline$S_{v}\left(120^{\prime \prime}\right)$ & Jy & 0.46 & $4.30(4.34)$ & 2.39 & 15.38 & 0.80 & $5.49(4.51)$ & 3.85 & 15.38 \\
\hline$S_{v}$ (int) & Jy & 0.43 & $4.56(4.62)$ & 2.09 & 17.26 & 0.56 & $5.84(4.78)$ & 4.65 & 17.26 \\
\hline$T_{\mathrm{mb}}(1,1)$ & $\mathrm{K}$ & 0.33 & $1.76(0.96)$ & 1.69 & 4.15 & 0.80 & $2.00(0.97)$ & 1.75 & 4.15 \\
\hline$T_{\mathrm{mb}}(2,2)$ & K & 0.27 & $0.82(0.41)$ & 0.69 & 1.72 & 0.29 & $0.92(0.44)$ & 0.83 & 1.72 \\
\hline$T_{\mathrm{ex}}(1,1)$ & $\mathrm{K}$ & 3.04 & $7.27(5.42)$ & 6.14 & 32.27 & 3.88 & $7.78(5.60)$ & 6.45 & 32.27 \\
\hline$T_{K}$ & $\mathrm{~K}$ & 12.49 & $19.39(4.89)$ & 18.69 & 32.27 & 13.39 & $20.56(5.08)$ & 19.47 & 32.27 \\
\hline$\left(T_{K}-T_{\mathrm{ex}}\right)$ & $\mathrm{K}$ & 0.00 & $12.13(4.61)$ & 13.05 & 21.83 & 0.00 & $12.78(4.48)$ & 13.24 & 21.83 \\
\hline$v_{\mathrm{LSR}}$ & $\mathrm{km} \mathrm{s}^{-1}$ & -1.00 & $6.83(2.96)$ & 7.85 & 10.17 & -0.53 & $6.93(2.76)$ & 7.85 & 10.12 \\
\hline$\sigma_{v}$ & $\mathrm{~km} \mathrm{~s}^{-1}$ & 0.35 & $0.69(0.27)$ & 0.58 & 1.31 & 0.38 & $0.75(0.27)$ & 0.71 & 1.31 \\
\hline$a$ & $\mathrm{~km} \mathrm{~s}^{-1}$ & 0.21 & $0.26(0.03)$ & 0.26 & 0.34 & 0.22 & $0.27(0.03)$ & 0.26 & 0.34 \\
\hline$\sigma_{\mathrm{NT}}$ & $\mathrm{km} \mathrm{s}^{-1}$ & 0.34 & $0.68(0.27)$ & 0.58 & 1.30 & 0.37 & $0.74(0.27)$ & 0.70 & 1.30 \\
\hline$\tau(1,1)$ & $\ldots$ & 0.05 & $1.12(0.72)$ & 1.16 & 3.08 & 0.08 & $1.02(0.70)$ & 0.80 & 3.08 \\
\hline$M_{\mathrm{iso}}\left(120^{\prime \prime}\right)$ & $M_{\odot}$ & 31 & $230(180)$ & 150 & 610 & 47 & $280(180)$ & 190 & 610 \\
\hline$M_{\text {iso }}$ (int) & $M_{\odot}$ & 28 & $250(210)$ & 150 & 850 & 33 & $300(220)$ & 230 & 850 \\
\hline$M_{\mathrm{vir}}$ (int) & $M_{\odot}$ & 22 & $190(160)$ & 150 & 560 & 33 & $220(160)$ & 190 & 560 \\
\hline$M_{\mathrm{vir}} / M_{\mathrm{iso}}(\mathrm{int})$ & & 0.23 & $1.00(0.95)$ & 0.70 & 4.37 & 0.23 & $0.96(0.93)$ & 0.65 & 4.37 \\
\hline$n_{p}($ int $)$ & $10^{3} \mathrm{~cm}^{-3}$ & 1.37 & $5.91(4.29)$ & 4.74 & 16.96 & 1.37 & $6.23(4.42)$ & 4.81 & 16.96 \\
\hline$\Sigma($ int $)$ & $\mathrm{g} \mathrm{cm}^{-2}$ & 0.018 & $0.048(0.030)$ & 0.041 & 0.127 & 0.019 & $0.055(0.032)$ & 0.045 & 0.127 \\
\hline$n_{\mathrm{ex}}$ & $10^{3} \mathrm{~cm}^{-3}$ & 0.49 & $7.96(6.79)$ & 6.65 & 29.90 & 2.07 & $9.60(7.05)$ & 8.29 & 29.90 \\
\hline$n_{p}(\mathrm{int}) / n_{\mathrm{ex}}$ & & 0.03 & $1.50(1.00)$ & 1.25 & 5.22 & 0.24 & $1.73(1.00)$ & 1.80 & 5.22 \\
\hline$N_{\mathrm{NH}_{3}}$ & $10^{13} \mathrm{~cm}^{-2}$ & 1.41 & $29.68(23.61)$ & 23.13 & 75.76 & 4.18 & $34.74(23.93)$ & 29.24 & 75.76 \\
\hline$N_{\mathrm{H}_{2}}$ (int) & $10^{21} \mathrm{~cm}^{-2}$ & 3.75 & $10.27(6.52)$ & 8.78 & 27.13 & 4.08 & $11.71(6.94)$ & 9.69 & 27.13 \\
\hline$N_{\mathrm{NH}_{3}} / N_{\mathrm{H}_{2}}$ (int) & $10^{-8}$ & 0.14 & $3.00(2.41)$ & 2.34 & 10.60 & 0.89 & $3.08(2.04)$ & 2.36 & 8.29 \\
\hline
\end{tabular}

Note.

${ }^{\text {a }}$ Standard deviation is given in parentheses.

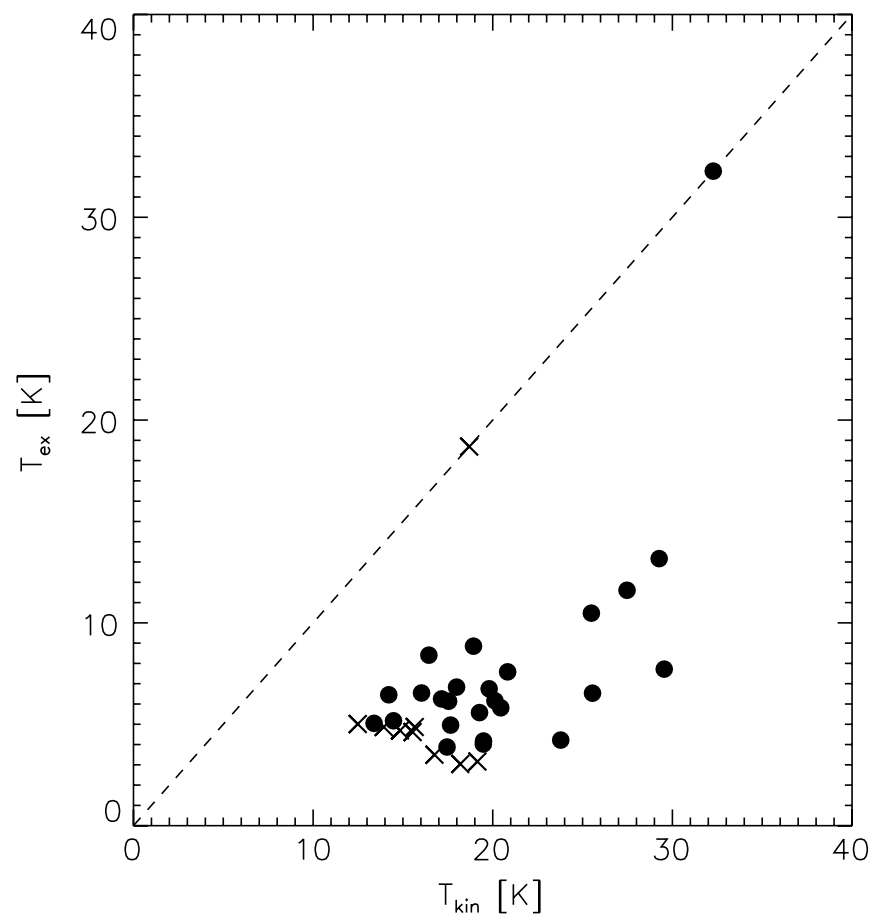

Figure 17. Excitation temperature of the $\mathrm{NH}_{3}(1,1)$ transition vs. kinetic temperature for the full sample. The filled circles mark subset 1 , and crosses mark sources with upper limits in $T_{\text {kin }}$. The dashed line marks a one-to-one correlation but is not a fit to the data.

set to unity. A lower excitation temperature could be artificially induced by overestimating the beam filling factor.
Another possible explanation is the density in the region. The level populations are determined by both collisional and radiative processes which depend on the local density. Pavlyuchenkov et al. (2008) study the conditions under which molecular rotational lines form using radiative transfer simulations of $\mathrm{CO}(J=2-1)$ and $\mathrm{HCO}^{+}(J=1-0)$, and define a thermalization density $n_{\mathrm{th}}$, where $T_{\mathrm{ex}}$ is within $5 \%$ of $T_{K}$. At densities greater than $n_{\text {th }}$, collisions regulate the level populations by both exciting and de-exciting molecules. A commonly used measure is the critical density, defined by $n_{\text {crit }}=A / \gamma$, where $A$ is the spontaneous emission rate and $\gamma$ is the collision rate coefficient. For low-frequency transitions, such as the $\mathrm{NH}_{3}$ inversion lines, collisions are negligible for $n<n_{\text {crit }}$, and the excitation temperature is set by the cosmic background radiation. At intermediate densities where $n_{\text {crit }}<n<n_{\text {th }}$, the excitation temperature is set by both collisions and radiation and is equal to neither the background radiation temperature nor the kinetic temperature.

Using a large velocity gradient (LVG) code (Snell et al. 1984), we calculate $T_{\mathrm{ex}}$ as a function of density for a gas temperature of $T_{K}=20 \mathrm{~K}$, with $N\left(\mathrm{NH}_{3}\right)=10^{14.5} \mathrm{~cm}^{-2}$, over a density range of $100-10^{8} \mathrm{~cm}^{-3}$. We assume a line width of $0.75 \mathrm{~km} \mathrm{~s}^{-1}$ to include radiative trapping. Figure 18 plots $T_{\mathrm{ex}}$ as a function of volume density for the $\mathrm{NH}_{3}(1,1)$ transition.

From these models, we find $n_{\text {th }}=210 n_{\text {crit }}$ for the $\mathrm{NH}_{3}(1,1)$ transition. The large difference in $n_{\text {crit }}$ and $n_{\text {th }}$ for the $\mathrm{NH}_{3}$ transitions is due to the low Einstein A coefficient and the importance of stimulated processes to the level populations. For comparison, Evans (1989) finds $n\left(T_{\mathrm{ex}}=0.9 T_{K}\right)=1.33 n_{\text {crit }}$ for the CS(3-2) transition. Although this criterion is less stringent than $n_{\text {th }}$, it demonstrates the differences between the lowfrequency radio transitions and higher frequency submillimeter 


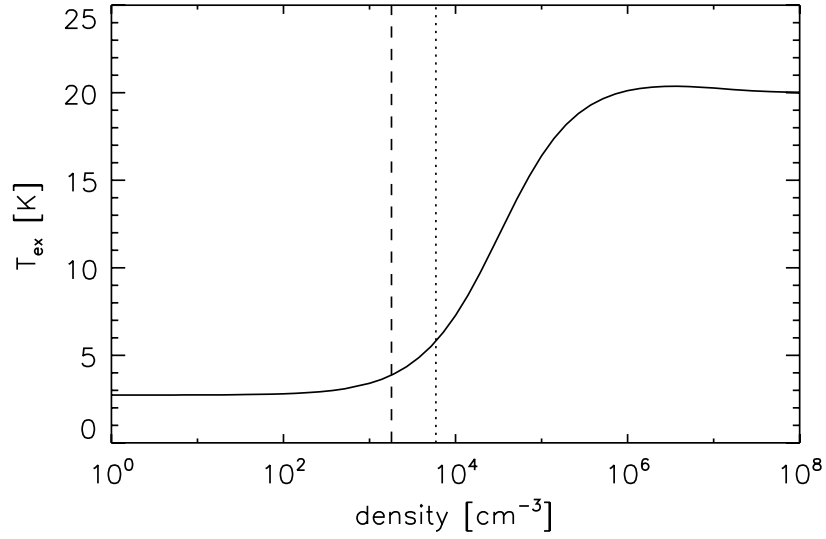

Figure 18. Excitation temperature vs. volume density for $\mathrm{NH}_{3}(1,1)$ for $T_{K}=$ $20 \mathrm{~K}$. Vertical dashed line denotes $n=n_{\text {crit }}(1,1)$, and the dotted line denotes the mean particle density found for subset 1 . The LVG code predicts an excitation temperature of $6.3 \mathrm{~K}$ at the mean density of our sample. Our mean excitation temperature is $7.3 \mathrm{~K}$.

transitions. The low-frequency transitions, such as the $\mathrm{NH}_{3}$ transitions, require a density much higher than the critical density before $T_{\mathrm{ex}}$ begins to approach $T_{K}$.

Although the volume-averaged densities derived from the millimeter continuum are approximately equal to the critical densities for both the $\mathrm{NH}_{3}(1,1)$ and $(2,2)$ transitions $(1.8 \times$ $10^{3} \mathrm{~cm}^{-3}$ and $2.1 \times 10^{3} \mathrm{~cm}^{-3}$, respectively; Tielens 2005), the densities are not high enough for the gas to be completely thermalized, and $T_{\mathrm{ex}}<T_{K}$ as expected at densities near the critical density.

\section{7. $\mathrm{NH}_{3}$ Excitation Density}

If we assume the gas is not in LTE, we can define the excitation density, $n_{\mathrm{ex}}$, as the density required to produce level populations described by $T_{\mathrm{ex}}$ for a given gas kinetic temperature. We calculate $n_{\mathrm{ex}}$ from $T_{K}, T_{\mathrm{ex}}$, and $\tau$ via the balance of a twolevel system. The excitation density is given by (Foster et al. 2009; Swift et al. 2005; Caselli et al. 2002)

$$
n_{\mathrm{ex}}=\frac{k\left(J\left(T_{\mathrm{ex}}\right)-J\left(T_{\mathrm{cmb}}\right)\right)}{h v_{(1,1)}\left(1-J\left(T_{\mathrm{ex}}\right) / J\left(T_{K}\right)\right)} n_{\mathrm{crit}} \beta,
$$

where

$$
J(T)=\frac{h v_{(1,1)}}{k\left(1-e^{-h v_{(1,1)} / k T}\right)} .
$$

$T_{\mathrm{cmb}}=2.73$ and the escape probability is given by $\beta=$ $\left(1-e^{-\tau}\right) / \tau$, where we take $\tau=\tau_{(1,1)} \times 0.233$ which is the maximum optical depth in a single hyperfine line of the $\mathrm{NH}_{3}(1,1)$ transition.

The excitation densities are listed in Column 6 of Table 7 . We cannot calculate $n_{\text {ex }}$ for the two sources where we set $T_{\mathrm{ex}}=T_{K}$ (see Section 6.6). The excitation densities for subset 1 range from $2.1 \times 10^{3} \mathrm{~cm}^{-3}$ to $29.9 \times 10^{3} \mathrm{~cm}^{-3}$ with a mean of $9.6 \pm 7.1 \times 10^{3} \mathrm{~cm}^{-3}$. Figure 19 shows a comparison of the $\mathrm{NH}_{3}$ excitation density and the volume-averaged density $\left(n_{p}\right)$ from the millimeter data. The dashed line marks a one-to-one correlation, and is not a fit to the data. Although there is considerable spread, $n_{p}$ is in good agreement with the excitation density. The mean ratio of $n_{p}$ (int) to excitation density is $1.7 \pm 1.0$ with a median of 1.8 for subset 1 . The agreement between $n_{\mathrm{ex}}$ and $n_{p}$ provide additional support that the $\mathrm{NH}_{3}$ and continuum emission trace the same physical region.

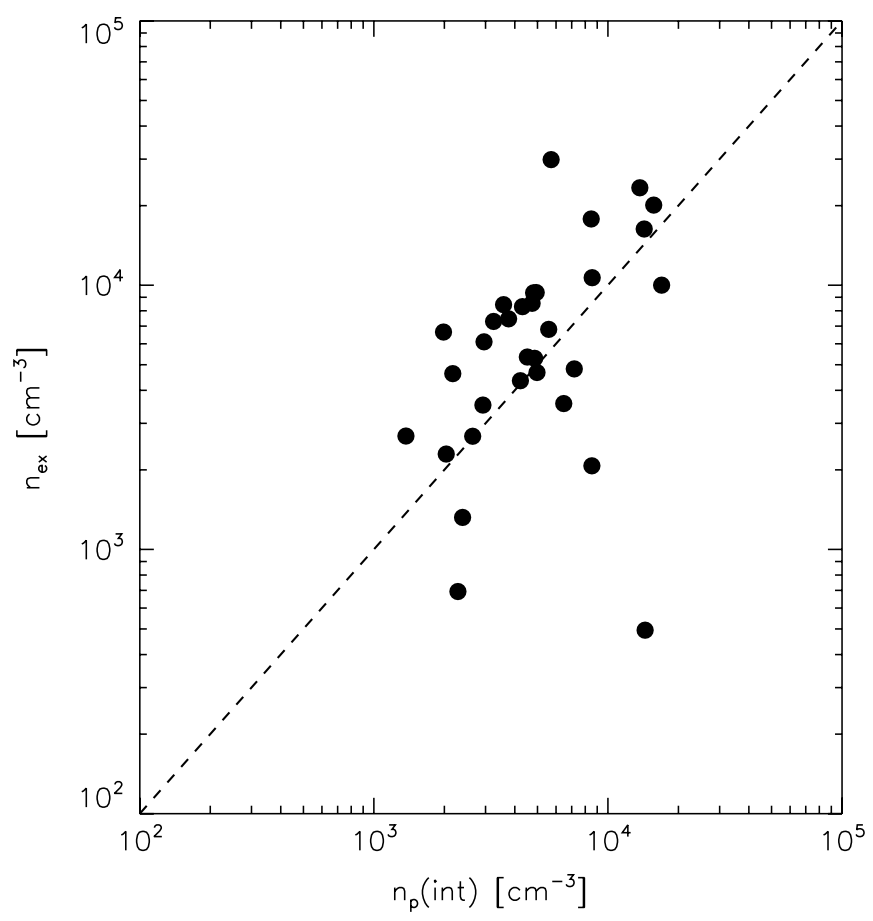

Figure 19. $\mathrm{NH}_{3}$ excitation density, $n_{\mathrm{ex}}$, vs. $n_{p}$ (int). The dashed line marks a one-to-one correlation and is not a fit to the data. The mean volume densities are in good agreement with the $\mathrm{NH}_{3}$ excitation densities.

\subsection{Column Densities}

The column density per beam of $\mathrm{NH}_{3}$ in the $(1,1)$ state can be calculated from the observed parameters via the following equation:

$$
N(1,1)=2 \frac{8 \pi v_{(1,1)}^{2}}{c^{2}} \frac{g_{1}}{g_{2}} \frac{1}{A_{(1,1)}}\left[1-e^{\frac{h v_{(1,1)}}{k T_{\mathrm{ex}}}}\right]^{-1} \int \tau(v) d \nu,
$$

where

$$
\begin{aligned}
\int \tau(v) d v & =\tau(1,1) \int d v \sum_{i} s_{i} \exp ^{-\frac{\left(v-v_{i}-v_{v_{L S R}}\right)^{2}}{2 \sigma_{i}^{2}}} \\
& =\sqrt{2 \pi} \frac{\sigma_{v}}{c} v_{(1.1)} \tau(1,1)
\end{aligned}
$$

and $v_{(1,1)}$ is the central frequency of the $(1,1)$ transition, $\tau_{(1,1)}$ is the total optical depth in the $(1,1)$ transition, $g_{1}$ and $g_{2}$ are the statistical weights of the upper and lower levels of the $(1,1)$ inversion transition and are equal, $A_{(1,1)}=1.68 \times 10^{-7} \mathrm{~s}^{-1}$ is the Einstein A coefficient for the transition (Pickett et al. 1998), and $\sigma_{v}$ is the Gaussian 1/e line width. The leading factor of 2 in Equation (12) assumes an equal number of $\mathrm{NH}_{3}$ molecules in each state of the inversion transition, which is justified since the states have equal statistical weight and are separated in energy by $0.05 \mathrm{~K}$. The total optical depth in the $(1,1)$ transition is split over the 18 hyperfine components, which is expressed in the summation in Equation (13). Here $s_{i}$ is the weight of each hyperfine component, $v_{i}$ is the frequency of each hyperfine component, and $\sigma_{i}$ is the Gaussian 1/e width of each hyperfine component.

The total $\mathrm{NH}_{3}$ column density, $N_{\mathrm{NH}_{3}}$, can be calculated by scaling $N(1,1)$ by $Z / Z(J=1)$ where $Z$ is the partition function given in Equations (2) and (3), $Z(J=1)$ is the population in only the $J=1$ level, and we truncate the summation at 50 terms. The $\mathrm{NH}_{3}$ column densities are given in Column 8 of Table 7 . 
$N_{\mathrm{NH}_{3}}$ ranges from $4.2 \times 10^{13} \mathrm{~cm}^{-2}$ to $75.8 \times 10^{13} \mathrm{~cm}^{-2}$ with a mean of $3.5 \times 10^{14} \mathrm{~cm}^{-2}$ for subset 1 .

We can also calculate the column density of $\mathrm{H}_{2}$ via the millimeter dust observations. From the gas and dust mass within a given aperture, we can calculate $N_{\mathrm{H}_{2}}$ by

$$
N_{\mathrm{H}_{2}}=\frac{M_{\text {iso }}}{\mu_{\mathrm{H}_{2}} m_{H} \pi R_{\mathrm{obj}}^{2}},
$$

where $\mu_{\mathrm{H}_{2}}=2.8 . N_{\mathrm{H}_{2}}$ is listed in Column 9 of Table 7 . $N_{\mathrm{H}_{2}}$ ranges from $4.1 \times 10^{21} \mathrm{~cm}^{-2}$ to $27.1 \times 10^{21} \mathrm{~cm}^{-2}$, with a mean of $1.2 \pm 0.7 \times 10^{22} \mathrm{~cm}^{-2}$. Using the relationship between $\mathrm{H}_{2}$ column density and visual extinction of Bohlin et al. (1978) assuming $R_{V}=3.1, N_{\mathrm{H}_{2}} / A_{V}=9.4 \times 10^{20} \mathrm{~cm}^{-2}$ mag $^{-1}$, we find $A_{V}=12.4$ mag for the mean $\mathrm{H}_{2}$ column density of our sample. However, $R_{V}=3.1$ may not be an accurate description of the dust in these dense regions. Chapman et al. (2009) find that regions with $A_{K}>1 \mathrm{mag}\left(A_{V}>5\right.$ mag) are described well by a $R_{V}=5.5$ extinction model (Weingartner \& Draine 2001). Using this extinction model, $N_{\mathrm{H}_{2}} / A_{V}=\left(2 \times 1.086 C_{\text {ext }}\right)^{-1}$, where the factor of 2 converts $N_{\mathrm{H}}$ to $N_{\mathrm{H}_{2}}$ and $C_{\text {ext }}$ is the extinction cross section in the $V$ band. Using $N_{\mathrm{H}_{2}} / A_{V}=6.86 \times 10^{20} \mathrm{~cm}^{-2} \mathrm{mag}^{-1}$, we find $A_{V}=17.5$ for the mean column density of our sample.

We can obtain a measurement of the $\mathrm{NH}_{3}$ abundance by comparing the column densities of $\mathrm{NH}_{3}$ and $\mathrm{H}_{2}$. The abundances are presented in Column 10 of Table 7 . The abundance ranges from $0.89 \times 10^{-8}$ to $8.3 \times 10^{-8}$ with a mean of $3.1 \times 10^{-8}$ for subset 1 . These results are similar to the values derived for dense cores, e.g., Harju et al. $\left(1993 ; 3 \times 10^{-8}\right)$, Tafalla et al. $\left(2006 ; 2.8 \times 10^{-8}\right)$, and Foster et al. $\left(2009 ; 2 \times 10^{-8}\right)$.

\section{DISCUSSION}

The physical properties of the BGPS sources in the Gemini OB1 molecular cloud show that the majority of the sources are clumps from which entire stellar clusters will form. Characteristics of all properties are listed in Table 8 for both the full sample and subset 1 , where numbers in parentheses are the standard deviation rather than an error in the mean. Recall that clumps are typically characterized by masses ranging from 50 to $500 M_{\odot}$, radii of $0.3-3 \mathrm{pc}$, mean densities of $10^{3}-$ $10^{4} \mathrm{~cm}^{-3}$, and gas temperatures of 10-20 K (Bergin \& Tafalla 2007). In the Gemini OB1 region, we find a mean isothermal mass of $\left\langle M_{\text {iso }}\right.$ (int $\left.)\right\rangle=200 \pm 220 M_{\odot}$, a mean radius of $\langle R\rangle=0.56 \pm 0.19 \mathrm{pc}$, a mean density of $\left\langle n_{p}(\mathrm{int})\right\rangle=$ $(6.2 \pm 4.4) \times 10^{3} \mathrm{~cm}^{-3}$, and a mean gas kinetic temperature of $\left\langle T_{K}\right\rangle=20.6 \pm 5.1 \mathrm{~K}$. The mean properties of our BGPS sources fall squarely in the ranges of characteristic clump properties.

In contrast, the unresolved BPGS source in this region (ID No. 51) has properties within the range of the characteristic properties of cores. Recall that cores have masses from $0.5-5 M_{\odot}$, sizes of $0.03-0.2$, mean densities of $10^{4}-10^{5}$, and gas temperatures between 8 and $12 \mathrm{~K}$ (Bergin \& Tafalla 2007). Source No. 51 has $M_{\text {iso }}$ (int $)=44 \pm 13 M_{\odot}$, an upper limit for size of $R<0.17 \mathrm{pc}$, a mean density of $n_{p}($ int $)=2.9 \times 10^{3} \mathrm{~cm}^{-3}$, and an upper limit of gas kinetic temperature of $T_{K}<14.8 \mathrm{~K} . M_{\text {iso }}$ (int) is significantly above the range of core masses, but also falls below the range of characteristic clump masses. The mean density does not fall within the characteristic density range of cores, but the upper limit used for the source size could be the explanation. An unresolved source must have a size smaller than the beam size, so the beam size is artificially giving a lower density.
There are additional unresolved BPGS sources but they do not have corresponding $\mathrm{NH}_{3}$ pointings and were excluded from our analysis.

Further evidence that the BGPS sources are not directly forming a single massive star is in the mean surface density of the sample, $\langle\Sigma($ int $)\rangle=0.055 \pm 0.032 \mathrm{~g} \mathrm{~cm}^{-2}$. Krumholz \& McKee (2008) have shown that a surface density of at least $1 \mathrm{~g} \mathrm{~cm}^{-2}$ is required to avoid fragmentation and form a single massive star. Although the mean masses of our clumps are high enough to form a massive star given typical star formation efficiencies, the mean surface density is not high enough to prevent fragmentation and suggests that there should be significant substructure within these clumps. Substructure has been seen in higher resolution studies of some of the Gemini OB1 BGPS clumps and is discussed in detail in the following section.

\subsection{Comparison to Other Studies}

A comparison to the literature provides further evidence that the BGPS sources are likely the clumps from which stellar clusters will form. The BGPS sources often resolve into multiple smaller, higher density regions when observed at higher resolution with telescopes such as the Submillimeter Array (SMA), SCUBA on the James Clerk Maxwell telescope (JCMT), and SHARC on the CSO. Densities calculated from 1.3 $\mathrm{mm}$ continuum SMA observations are on the order of $10^{6}-10^{7}$ $\mathrm{cm}^{-3}$ (Cyganowski et al. 2007), a factor of $10^{3}$ greater than the densities of the BGPS sources presented here. Models of observed spectral energy distributions (SED) require both a hot $(\sim 100 \mathrm{~K})$ and warm $(\sim 40 \mathrm{~K})$ dust component (Minier et al. 2005), again suggesting that the BGPS sources are the cooler, lower density clumps which contain hotter cores. When comparing our $1.1 \mathrm{~mm}$ emission to the large-scale $\mathrm{CO}$ emission, we find that the BGPS sources have $\mathrm{H}_{2}$ column densities a factor of 10 greater than the mean column density of the regions of the molecular cloud without star formation (Carpenter et al. 1995a). The resolved BGPS sources are typically clumps which are embedded in a molecular cloud and contain higher density, warmer cores while the unresolved BGPS sources are likely to be cores. Detailed comparisons for a few sources and the entire molecular cloud are presented below.

\subsubsection{2}

S252 corresponds to G188.948+00.883 (ID No. 26) in the BGPS. Minier et al. (2005, hereafter M05) observed methanol and $\mathrm{C}^{18} \mathrm{O}$ toward $\mathrm{S} 252$, and also modeled mid-IR to millimeter wavelength emission. Using SCUBA on the JCMT and SIMBA on the Sweedish ESO Submillimetre Telescope (SEST), they resolve S252 into two millimeter sources, G188.95-MM1 and - MM2. Models fit to the spectral energy distributions require a warm extended envelope (42 and $50 \mathrm{~K}$ ) for each source and a hot central component $(150 \mathrm{~K})$ for MM1. The rotational temperature derived from their methanol observations is $58 \mathrm{~K}$. The temperatures derived from both SED fitting and molecular line observations are all significantly warmer than our $\mathrm{NH}_{3}$ kinetic temperature of $29 \mathrm{~K}$. In addition to warmer temperatures, M05 also derived a smaller mass than the BGPS. The BGPS does not resolve two sources here, and finds $M_{\text {iso }}$ (int $)=556 M_{\odot}$, while M05 finds a total mass for MM1 and MM2 of $155 M_{\odot}$ when scaled to our assumed distance of $2.1 \mathrm{kpc}$.

\subsection{2. $S 252 A$}

Mueller et al. (2002, hereafter M02) observed S252A in $350 \mu \mathrm{m}$ continuum emission using SHARC at the CSO, and 
modeled the observed SED including wavelengths ranging from $12 \mu \mathrm{m}$ to $1.1 \mathrm{~mm}$. They fit a power-law density distribution and determined a density normalized at a radius of $1000 \mathrm{AU}, n_{f}$. S252A in the M02 sample corresponds to G189.776+00.343 (ID No. 13) in our sample. M02 calculated an isothermal dust mass of $220 M_{\odot}$ within a $120^{\prime \prime}$ aperture when scaled to our assumed distance. We find $M_{\text {iso }}\left(120^{\prime \prime}\right)=373 \pm 40 M_{\odot}$ from the $1.1 \mathrm{~mm}$ emission. SHARC filtered out extended emission on a smaller scale than the BGPS, so the M02 observations detected the warmer, higher density, and lowermass substructure within the BGPS source. The $T_{\text {iso }}$ given by M02 was $30 \mathrm{~K}$, marginally warmer than the temperature derived from the $\mathrm{NH}_{3}$ observations, $T_{K}=26 \mathrm{~K}$.

In general, the M02 study included higher-mass, warmer sources than our sample. Their sample selection required the presence of strong water maser emission (Arcerti water maser catalog; Cesaroni et al. 1988) as a signpost of massive star formation. M02 found $\left\langle M_{\text {iso }}\left(120^{\prime \prime}\right)\right\rangle=2040 \pm 4410 M_{\odot}$, with a median of $400 M_{\odot}$ for their sample of 51 massive star-forming regions. In contrast, we find $\left\langle M_{\text {iso }}\left(120^{\prime \prime}\right)\right\rangle=280 \pm 180 M_{\odot}$, with a median of $190 M_{\odot}$. Our sources are approximately a factor of 10 less massive than the sample studied by M02.

\subsubsection{S255N and S255IR}

S255N corresponds to G192.581-00.043 (ID No. 45) in our sample, while S255IR corresponds to G192.596-00.051 (ID No. 47). We find $M_{\text {iso }}$ (int) $=397 \pm 32 M_{\odot}$ and $T_{K}=30 \mathrm{~K}$ for S255N, and $M_{\text {iso }}$ (int) $=540 \pm 43$ and $T_{K}=32 \mathrm{~K}$ for S255IR. We compare our BGPS and $\mathrm{NH}_{3}$ derived properties with studies by Zinchenko et al. (1997, hereafter Z97), M05, and Cyganowski et al. (2007, hereafter C07) in detail.

Z97 mapped $\mathrm{NH}_{3}(1,1)$ and $(2,2)$ emission from $\mathrm{S} 255$ with the $100 \mathrm{~m}$ Effelsberg Radio Telescope. They centered their map on an IRAS point source located between S255N and S255IR, and considered the emission from both to be part of a single source. They report observed values at two locations, one of which corresponds very well with S255N and our source G192.583-0.043. The observed $\mathrm{NH}_{3}$ properties between the two studies agree fairly well considering the $\mathrm{NH}_{3}$ positions differ slightly. For example, Z97 find $v_{\mathrm{lsr}}=8.65 \mathrm{~km} \mathrm{~s}^{-1}$ and $\sigma_{v}=\Delta v /(8 \ln 2)^{1 / 2}=1.02 \mathrm{~km} \mathrm{~s}^{-1}$, while we find $v_{\text {lsr }}=8.66 \mathrm{~km} \mathrm{~s}^{-1}$ and $\sigma_{v}=1.31 \mathrm{~km} \mathrm{~s}^{-1}$. Z97 find that in half of their sources, the $\mathrm{NH}_{3}$ line width increases toward the $\mathrm{NH}_{3}$ peak, which could account for the same radial velocity but different line widths. Both studies derive slightly different $T_{K}$, with Z97 finding $T_{K}=23 \mathrm{~K}$ and our study finding $T_{K}=30 \mathrm{~K}$ for S255N and $32 \mathrm{~K}$ for S255IR. The Z97 mass derived from the $\mathrm{NH}_{3}$ column density and a canonical abundance is $420 M_{\odot}$ when scaled to our assumed distance. Since this includes both S255N and S255IR, this mass should be compared to the sum of masses for the two BGPS sources, $M_{\text {iso }}($ int,tot $)=937 M_{\odot}$. Our total $M_{\text {iso }}$ (int) is comparable to the virial mass Z97 derive, $M_{\mathrm{vir}}(\mathrm{Z} 97)=1134 M_{\odot}$ when scaled to the maser parallax distance.

C07 observed S255N in $350 \mu \mathrm{m}$ continuum with SHARC on the CSO and $1.3 \mathrm{~mm}$ continuum with the SMA. S255NMM1 corresponds to G192.581-0.043 in our sample (ID No. 45). SHARC $\left(\Theta_{\mathrm{mb}}=15^{\prime \prime}\right)$ recovers only a single peak at the position of S255N-MM1, while the SMA (4".7 × 2".4 beam) clearly resolves three separate sources: SMA1, SMA2, and SMA3. The total beam-averaged mass of all three sources scaled to the parallax distance is $17-42 M_{\odot}$, where the range is due to uncertainty in the assumed dust temperature. The assumed temperature range for SMA1 is $40-100 \mathrm{~K}$, while the other two sources are likely between 20 and $40 \mathrm{~K}$ based on the presence of molecular emission in SMA1 and the lack thereof in SMA2 and SMA3. We find $T_{K}=29.5 \mathrm{~K}$ for source No. 45 in our sample, which is consistent with the range of temperatures for SMA2 and 3 and is just below the lower end of the temperature range for SMA1. The difference in the temperatures reflects the different material probed with the two sets of observations: the SMA observes the warm, high density structure within the cooler, lower density clumps to which the BGPS and GBT observations are sensitive.

C07 derived densities in the range of $10^{6}-10^{7} \mathrm{~cm}^{-3}$ from the SMA $1.3 \mathrm{~mm}$ continuum emission. These densities are $\sim 10^{3}$ times higher than the derived BGPS densities. With a smaller beam size, the SMA resolves out the low density material which the BPGS is sensitive to and is able to detect the smaller, higher density regions within the BGPS clump. Our calculated $n_{p}$ is a volume-averaged density which will underestimate the peak density of the regions where massive stars will form because a larger percentage of the volume is at the lower densities.

Similar to C07, M05 found that S255N requires a hot component of $106 \mathrm{~K}$ and a warm component of $44 \mathrm{~K}$ while S255IR requires a hot component of $225 \mathrm{~K}$ and a warm component of $42 \mathrm{~K}$ based on fitting models to the observed spectral energy distribution. M05 find $M_{\text {iso }}$ of 140 and $210 M_{\odot}$ for S255N and S255IR, respectively. Their masses are significantly smaller than our isothermal masses, which is consistent with the paradigm in which observations with a smaller beam size will resolve out the lower density emission and detect the substructure within the BGPS sources.

Chavarría et al. (2008) provide direct evidence that S255IR and S255N are forming a cluster. They used Spitzer IRAC and NIR data to identify sources with near- or mid-IR excesses. They identified a total of 10 clusters of YSOs as well as a population of distributed YSOs. The largest cluster they identified corresponds to both S255IR and S255N (ID Nos. 45 and 47 in our sample). They detected 140 stars with an IR excess, and assume the total cluster membership to be 488 sources after accounting for completeness and sources with no IR excess.

\subsection{4. $S 258$ and $G 192.719+00.043$}

There is also direct evidence of clusters forming in both S258 (G192.644+00.003; ID No. 48) and G192.719+00.043 (ID No. 49) Chavarría et al. (2008) identified 49 sources with IR excesses in S258, and estimate the total number of cluster members to be 186 after considering completeness limits and the number of potential sources without an IR excess. The cluster they identified in G192.719+00.043 includes 22 detected members and is estimated to include 44 total members.

\subsubsection{Gemini OB1 Molecular Cloud}

Carpenter et al. (1995a, hereafter C95a) surveyed $32 \mathrm{deg}^{2}$ of the Gemini OB1 molecular cloud complex in ${ }^{12} \mathrm{CO}(J=1-0)$ and ${ }^{13} \mathrm{CO}(J=1-0)$. The detailed $\mathrm{CO}$ maps yield a wealth of physical properties, which provide an independent check of the properties determined in this study. C95a separated the molecular cloud into seven subregions marked by bright extended ${ }^{13} \mathrm{CO}$ emission. Subregions $1-3$ are associated with optical $\mathrm{H}_{\mathrm{II}}$ regions and correspond to groups $1-3$ in this paper. Subregion 6 contains a Herbig Ae/Be star HD250550, but is not included in our BGPS image. The remaining subregions have no optical evidence of star formation. 
Along the lines of sight with a $5 \sigma$ detection in ${ }^{13} \mathrm{CO}, \mathrm{C} 95 \mathrm{a}$ assume the ${ }^{12} \mathrm{CO}$ is optically thick and can determine $T_{K}$. For subregions 4-7 with no star formation, $T_{K}$ is typically $6-8 \mathrm{~K}$. In contrast, in subregions 1-3 with active star formation and optical HII regions the bulk of the material is found to be 6-8 $\mathrm{K}$ but the distribution has a warm tail reaching up to $T_{K}=25 \mathrm{~K}$. The kinetic temperatures $(\sim 10-30 \mathrm{~K})$ we derive from the $\mathrm{NH}_{3}$ spectra are consistent with the warmer $\mathrm{CO}$ lines of sight where active star formation is likely occurring. C95a also determine the column density of $\mathrm{H}_{2}$ along lines of sight with a $5 \sigma$ detection of ${ }^{13} \mathrm{CO}$. The subregions without star formation have $\left\langle N_{\mathrm{H}_{2}}\right\rangle=(1.0-1.3) \times 10^{21} \mathrm{~cm}^{-2}$, while the cloud as a whole ranges from $1.2 \times 10^{20} \mathrm{~cm}^{-2}$ to $6.4 \times 10^{22} \mathrm{~cm}^{-2}$. This is again consistent with our study, where we find $\left\langle N_{\mathrm{H}_{2}}\right.$ (int) $\rangle=1.2 \times$ $10^{22} \mathrm{~cm}^{-2}$.

For the subregions which correspond to our groups, we can determine the fraction of mass in dense clumps. To do so, we compare $M_{\text {iso }}$ (int) with $M_{\mathrm{LTE}}$ from C95a. The total mass of dense millimeter clumps is 1835,4549 , and $1973 M_{\odot}$ for groups $1-3$, respectively. The corresponding $M_{\mathrm{LTE}}$ for subregions $1-3$ are $3.5 \times 10^{4}, 5.2 \times 10^{4}$, and $4.3 \times 10^{4} M_{\odot}$. We find 5.2\%, $8.7 \%$, and $4.6 \%$ of the total mass is in the dense clumps for subregions and groups 1-3, respectively. C95a find that $10 \%$ of the molecular mass traced by $\mathrm{CO}$ is contained in lines of sight with $\mathrm{H}_{2}$ column densities greater than $10^{22} \mathrm{~cm}^{-2}$, similar to the mean column density of our clumps. This percentage is also similar to nearby, low-mass, star-forming molecular clouds Perseus, Ophiuchus, and Serpens, where $<10 \%$ of the total mass is in dense cores (Enoch et al. 2007). The fraction of mass found in the dense regions associated with star formation is very similar using different tracers as well as in high-mass and lowmass star-forming regions, two very different environments.

\subsection{What is a BGPS Source?}

The BGPS is detecting thousands of sources which span a vast range of distances, and it is important to understand the biases and trends inherent in the data. In contrast to the inner Galaxy BGPS sources, the Gemini OB1 molecular cloud provides an excellent opportunity to characterize the clump properties without large uncertainties in distances. Two neighboring sources in the inner Galaxy could be many kpc apart along the line of sight, whereas the Gemini OB1 sources are all located at the same distance. Since we have an accurate measure of the distance to the Gemini OB1 sample, we will use it as a starting point to understand the effects of distance on the derived properties.

The detection criterion for a BGPS source is equivalent to a $5 \sigma$ detection within an area the size of a beam (Rosolowsky et al. 2009). This results in a minimum mass per beam, below which the emission will be lost in the noise or removed during the sky cleaning process. This minimum mass is given by Equation (5), with $S_{v}=5 \sigma$, where $\sigma$ is the rms noise per pixel in units of Jy beam ${ }^{-1}$ in the BGPS image, $D$ is the distance, $\kappa_{v}$ is the dust opacity per gram of gas and dust, and $B_{v}\left(T_{d}\right)$ is the Planck function evaluated at the dust temperature. Thus, the BGPS is subject to the Malmquist bias: as the distance increases, the minimum mass to which the BGPS is sensitive also increases.

The minimum volume-averaged particle density to which the BGPS is sensitive is given by

$$
n_{p, \min }=\frac{15 \sigma}{4 \pi \mu m_{H} \kappa_{v} B_{v}\left(T_{d}\right) \Theta_{\mathrm{mb}}^{3} D} \propto \frac{1}{\Theta_{\mathrm{mb}}^{3} D},
$$

where $\mu=2.37, m_{H}$ is the mass of a hydrogen atom, and $\Theta_{\mathrm{mb}}$ is the FWHM of the CSO beam at $1.1 \mathrm{~mm}\left(33^{\prime \prime}\right)$. As the distance increases, the minimum mass increases (Equation (5)), and the minimum density decreases (Equation (15)) for sources smaller than the beam size, quantifying how BGPS observations are subject to Malmquist bias and beam dilution, respectively. Similarly, if we allow the aperture size for photometry to vary at a constant distance, larger sources will have lower densities.

For the Gemini OB1 molecular cloud observations, $\sigma=$ $0.37 \mathrm{Jy}_{\text {beam }^{-1}}$, requiring $S_{\nu}($ beam $)=0.35 \mathrm{Jy}$ for a $5 \sigma$ detection. The corresponding minimum mass is $M_{\min }=20 M_{\odot}$ at a distance of $2.1 \mathrm{kpc}$ and $T_{D}=20 \mathrm{~K}$. Thus, the minimum density required to detect a beam-sized source after deconvolution in the Gemini OB1 molecular cloud is $n_{p, \min } \approx 10^{4} \mathrm{~cm}^{-3}$. Sources larger than the beam size can have densities lower than $10^{4} \mathrm{~cm}^{-3}$. The smallest BGPS sources could have the highest densities and may be the cores which will form individual stars, multiple star systems, or small groups, whereas the larger sources will have generally lower densities and are likely to be the clumps which will form stellar clusters.

In the inner Galaxy, the largest distance will greatly exceed that of the Gemini OB1 molecular cloud and the minimum density will be significantly lower. At distances greater than approximately $2 \mathrm{kpc}$, the BGPS is only able to detect clumps which will form stellar clusters, and not individual cores. Since the Gemini OB1 molecular cloud lies at $2.1 \mathrm{kpc}$, we may detect some cores as well as clumps.

\section{SUMMARY}

We present the image and catalog of the Gemini OB1 molecular cloud complex from the $1.1 \mathrm{~mm}$ BGPS. We observed $\mathrm{NH}_{3}(1,1),(2,2)$, and $(3,3)$ inversion transitions and $22 \mathrm{GHz} \mathrm{H} \mathrm{H}_{2} \mathrm{O}$ maser transitions toward 37 BGPS sources, as well as $\mathrm{NH}_{3}(4,4)$ and $\mathrm{C}_{2} \mathrm{~S}$ toward 10 sources with the GBT. We have obtained a detection $\geqslant 5 \sigma$ in the $(1,1)$ transition of 34 of the 37 BGPS sources, and a detection $\geqslant 5 \sigma$ in the $(1,1)$ and $(2,2)$ transitions in 25 of the 34 BGPS sources.

We use a seeded watershed algorithm to extract the millimeter continuum sources from the BGPS maps. We find a mean FWHM of sources of $110^{\prime \prime}$, or $1.12 \mathrm{pc}$ at a distance of $2.1 \mathrm{kpc}$. We estimate the gas physical properties by fitting a simple model to all $\mathrm{NH}_{3}$ transitions simultaneously. From these fits, we find $\left\langle T_{K}\right\rangle=20 \mathrm{~K}$, and velocity dispersions ranging from 0.4 to $1.3 \mathrm{~km} \mathrm{~s}^{-1}$. We calculate the non-thermal velocity dispersion, compare it to the expected thermal sound speed, and find a ratio of $\sigma_{\mathrm{NT}}$ to $a$ ranging from 1.5 to 4.2 with a mean of $2.6 \pm 0.8$. The velocity dispersions of all sources are dominated by non-thermal motions.

We calculate the isothermal mass for each millimeter source based on $S_{\nu}\left(120^{\prime \prime}\right)$ and $S_{v}$ (int) assuming $T_{D}=T_{K}$ from the $\mathrm{NH}_{3}$ observations. We find $\left\langle M_{\text {iso }}\left(120^{\prime \prime}\right)\right\rangle=230 \pm 180 M_{\odot}$ and $\left\langle M_{\text {iso }}(\right.$ int $\left.)\right\rangle=250 \pm 210 M_{\odot}$, where the error is the standard deviation of the sample. We also calculate the virial mass within the source size derived by the source extraction algorithm under the assumption that the $\mathrm{NH}_{3}$ emission traces the millimeter emission well. We find $\left\langle M_{\text {vir }}\right\rangle=190 \pm 160 M_{\odot}$. The mean $M_{\text {vir }}$ to $M_{\text {iso }}$ ratio is $1.0 \pm 0.95$. The total isothermal mass for the portion of the Gemini OB1 molecular cloud we have mapped is $8400 M_{\odot}$, which represents $6.5 \%$ of the total mass seen in CO observations.

We also calculate the volume-averaged densities and find $\left\langle n_{p}(\mathrm{int})\right\rangle=5.9 \pm 4.3 \times 10^{3} \mathrm{~cm}^{-3}$, with the highest density being $n_{p, \text { max }}(\mathrm{int})=1.7 \times 10^{4} \mathrm{~cm}^{-3}$. The mean surface density of the full sample is $\langle\Sigma(\mathrm{int})\rangle=0.048 \pm 0.030 \mathrm{~g} \mathrm{~cm}^{-2}$. We 
calculate both $\mathrm{NH}_{3}$ and $\mathrm{H}_{2}$ column densities and find mean values of $3.0 \times 10^{14} \mathrm{~cm}^{-2}$ and $1.0 \times 10^{22} \mathrm{~cm}^{-2}$, respectively. We find a mean $\mathrm{NH}_{3}$ abundance of $3.0 \times 10^{-8}$ relative to $\mathrm{H}_{2}$.

The mean sizes, densities, kinetic temperatures, and surface densities of the BGPS sources suggest they are cluster-forming clumps that contain the cores that will form individual or small multiple systems.

We thank the anonymous referee, whose comments and suggestions have greatly improved this paper. The Caltech Submillimeter Observatory (CSO) is operated by Caltech under a contract from the National Science Foundation. The Green Bank Telescope (GBT) is operated by the National Radio Astronomy Observatory (NRAO). The National Radio Astronomy Observatory is a facility of the National Science Foundation, operated under cooperative agreement by Associated Universities, Inc. We acknowledge the staff and day crew of the CSO, and also the GBT operators and staff for their assistance. The BGPS is supported by the National Science Foundation through NSF grant AST-0708403. The first observing runs for the BGPS and the GBT observing runs were supported by travel funds provided by the National Radio Astronomy Observatory. J.A. was supported by a Jansky Fellowship from the NRAO. Support for the development of Bolocam was provided by NSF grants AST-9980846 and AST-0206158. M.K.D. and N.J.E. were supported by NSF grant AST-0607793 to the University of Texas at Austin. E.R. was supported by NSF grant AST-0502605 and a Discovery Grant from NSERC of Canada. C.J.C. was partially supported during this work by a National Science Foundation Graduate Research Fellowship. This research has made use of the NASA Astrophysics Data System (ADS) Abstract Service and of the SIMBAD database, operated at CDS, Strasbourg, France.

\section{REFERENCES}

Aguirre, J., et al. 2010, ApJ, submitted

Bally, J., et al. 2010, ApJ, submitted

Barbaro, G., Dallaporta, N.. \& Fabris, G. 1969, Ap\&SS, 3, 123

Bergin, E. A., \& Tafalla, M. 2007, ARA\&A, 45, 339

Bertoldi, F. \& McKee, C. F. 1992, ApJ, 395, 140

Beuther, H., Walsh, A., Schilke, P., Sridharan, T. K., Menten, K. M., \& Wyrowski, F. 2002, A\&A, 390, 289

Bohlin, R. C., Savage, B. D., \& Drake, J. F. 1978, ApJ, 224, 132

Carpenter, J. M., Snell, R. L., \& Schloerb, F. P. 1995a, ApJ, 445, 246

Carpenter, J. M., Snell, R. L., \& Schloerb, F. P. 1995b, ApJ, 450, 201

Caselli, P., Benson, P. J., Myers, P. C., \& Tafalla, M. 2002, ApJ, 572, 238

Cesaroni, R., Palagi, F., Felli, M., Catarzi, M., Comoretto, G., di Francos, Giovanardi, C., \& Palla, F. 1988, A\&AS, 76, 445

Chambers, E. T., Jackson, J. M., Rathborne, J. M., \& Simon, R. 2009, ApJS, 181,360

Chapman, N. L., Mundy, L. G., Lai, S.-P., \& Evans, N. J. 2009, ApJ, 690, 496

Chavarría, L. A., Allen, L. E., Hora, J. L., Brunt, C. M., \& Fazio, G. G. 2008, ApJ, 682,445

Crawford, D., et al. 1955, ApJ, 121, 24

Cyganowski, C. J., Brogan, C. L., \& Hunter, T. R. 2007, AJ, 134, 346

Dame, T. M., et al. 1987, ApJ, 322, 706

Dobashi, K., Uehara, H., Kandori, R., Sakurai, T., Kaiden, M., Umemoto, T., \& Sato, F. 2005, PASJ, 57, 1

Elitzur, M., Hollenbach, D. J., \& McKee, C. F. 1989, ApJ, 346, 983

Enoch, M. L., Glenn, J., Evans, N. J., II, Sargent, A. I., Young, K. E., \& Huard, T. L. 2007, ApJ, 666, 982

Evans, N. J., II 1989, RevMexAA, 18, 21

Evans, N. J., II, Beckwith, S., \& Blair, G. N. 1977, ApJ, 217, 448

Evans, N. J., II, Rawlings, J. M. C., Shirley, Y. L., \& Mundy, L. G. 2001, ApJ, 557,193

Foster, J. B., Rosolowsky, E. W., Kauffmann, J., Pineda, J. E., Borkin, M. A., Caselli, P., Myers, P. C., \& Goodman, A. A. 2009, ApJ, 696, 298
Friesen, R. K., Di Francesco, J., Shirley, Y. L., \& Myers, P. C. 2009, ApJ, 697, 1457

Georgelin, Y. M., Georgelin, Y. P., \& Roux, S. 1973, A\&A, 25, 337

Glenn, J., et al. 2003, Proc. SPIE, 4855, 30

Goldreich, P., \& Kwan, J. 1974, ApJ, 189, 441

Grasdalen, G. L.. \& Carrasco, L. 1975, A\&A, 43, 259

Haig, D. J., et al. 2004, Proc. SPIE, 5498, 78

Hardie, R. H., Seyfert, C. K., \& Gulledge, I. S. 1960, ApJ, 132, 36

Harju, J., Walmsley, C. M., \& Wouterloot, J. G. A. 1993, A\&AS, 98, 51

Ho, P. T. P., \& Townes, C. H. 1983, ARA\&A, 21, 239

Huang, Y.-L., \& Thaddeus, P. 1986, ApJ, 309, 804

Humphreys, R. M. 1978, ApJS, 38, 309

Jackson, J. M., Finn, S. C., Rathborne, J. M., Chambers, E. T., \& Simon, R. 2008, ApJ, 680, 349

Kömpe, C., Joncas, G., Baudry, A., \& Wouterloot, J. G. A. 1989, A\&A, 221, 295

Krumholz, M. R., \& McKee, C. F. 2008, Nature, 451, 1082

Kumar, M. S. N., \& Grave, J. M. C. 2007, A\&A, 472, 155

Kumar, M. S. N., Keto, E., \& Clerkin, E. 2006, A\&A, 449, 1033

Lovas, F. J., \& Dragoset, R. 2003, Recommended Rest Frequencies for Observed Interstellar Molecular Microwave Transitions (Revised ed.; Washington, DC: National Bureau of Standards)

Minier, V., Burton, M. G., Hill, T., Pestalozzi, M. R., Purcell, C. R., Garay, G., Walsh, A. J., \& Longmore, S. 2005, A\&A, 429, 945

Molinari, S., Brand, J., Cesaroni, R., \& Palla, F. 2000, A\&A, 355, 617

Morgan, W. W., Whitford, A. E., \& Code, A. D. 1953, ApJ, 118, 318

Motte, F., Bontemps, S., Schilke, P., Schneider, N., Menten, K. M., \& Broguière, D. 2007, A\&A, 476, 1243

Mueller, K. E., Shirley, Y. L., Evans, N. J., II, \& Jacobson, H. R. 2002, ApJS, 143,469

Ossenkopf, V., \& Henning, T. 1994, A\&A, 291, 943

Palla, F., Brand, J., Comoretto, G., Felli, M., \& Cesaroni, R. 1991, A\&A, 246, 249

Pavlyuchenkov, Y., Wiebe, D., Shustov, B., Henning, T., Launhardt, R., \& Semenov, D. 2008, ApJ, 689, 335

Pickett, H. M., Poynter, I. R. L., Cohen, E. A., Delitsky, M. L., Pearson, J. C., \& Muller, H. S. P. 1998, J. Quant. Spectrosc. Radiat. Transfer, 60, 883

Plume, R., Jaffe, D. T., \& Evans, N. J., II 1992, ApJS, 78, 505

Plume, R., Jaffe, D. T., Evans, N. J., II, Martin-Pintado, J., \& Gomez-Gonzalez, J. 1997, ApJ, 476, 730

Pohl, M., Englmaier, P., \& Bissantz, N. 2008, ApJ, 677, 283

Ramesh, B., \& Sridharan, T. K. 1997, MNRAS, 284, 1001

Rathborne, J. M., Jackson, J. M., \& Simon, R. 2006, ApJ, 641, 389

Reid, M. J., Menten, K. M., Brunthaler, A., Zheng, X. W., Moscadelli, L., \& Xu, Y. 2009, ApJ, 693, 397

Robitaille, T. P., et al. 2008, AJ, 136, 2413

Rosolowsky, E. W., Pineda, J. E., Foster, J. B., Borkin, M. A., Kauffmann, J., Caselli, P., Myers, P. C., \& Goodman, A. A. 2008, ApJS, 175, 509

Rosolowsky, E. W., et al. 2010, ApJS, 188, 123

Schuller, F., et al. 2009, A\&A, 504, 415

Sharpless, S. 1959, ApJS, 4, 257

Shirley, Y. L., Evans, N. J., II, \& Rawlings, J. M. C. 2002, ApJ, 575, 337

Shirley, Y. L., Evans, N. J., II, Young, K. E., Knez, C., \& Jaffe, D. T. 2003, ApJS, 149,375

Snell, R. L., Goldsmith, P. F., Erickson, N. R., Mundy, L. G., \& Evans, N. J., II 1984, ApJ, 276, 625

Soille, P. 1999, Morphological Image Analysis (Berlin: Springer)

Sridharan, T. K., Beuther, H., Schilke, P., Menten, K. M., \& Wyrowski, F. 2002, ApJ, 566, 931

Strelnitskii, V. S. 1984, MNRAS, 207, 339

Swift, J. J., Welch, W. J., \& Di Francesco, J. 2005, ApJ, 620, 823

Szymczak, M., Pillai, T. \& Menten, K. M. 2005, A\&A, 434, 613

Tafalla, M., Santiago-García, J., Myers, P. C., Caselli, P., Walmsley, C. M., \& Crapsi, A. 2006, A\&A, 455, 577

Takano, S., Nakai, N., \& Kawaguchi, K. 2002, PASJ, 54, 195

Tielens, A. G. G. M. 2005 (ed.), The Physics and Chemistry of the Interstellar Medium (Cambridge: Cambridge Univ. Press)

Weingartner, J. C., \& Draine, B. T. 2001, ApJ, 548, 296

Williams, J. P., Blitz, L., \& McKee, C. F. 2000, in Protostars and Planets IV, ed. V. Mannings, A. P. Boss, \& S. S. Russell (Tucson, AZ: Univ. Arizona Press), 97

Wood, D. O. S., \& Churchwell, E. 1989, ApJ, 340, 265

Yamamoto, S., Saito, S., Kawaguchi, K., Chikada, Y., Suzuki, H., Kaifu, N., Ishikawa, S.-I., \& Ohishi, M. 1990, ApJ, 361, 318

Zinchenko, I., Henning, T., \& Schreyer, K. 1997, A\&AS, 124, 385 